JOURNAL OF THE AMERICAN MATHEMATICAL SOCIETY

Volume 20, Number 1, January 2007, Pages 53-97

$\mathrm{S}$ 0894-0347(06)00532-7

Article electronically published on March 17, 2006

\title{
UNIVERSAL CHARACTERISTIC FACTORS AND FURSTENBERG AVERAGES
}

\author{
TAMAR ZIEGLER
}

\section{INTRODUCTION}

Averages of the form

$$
\frac{1}{N} \sum_{n=1}^{N} \prod_{j=1}^{k} f\left(T^{j n} x\right)
$$

were first introduced by Furstenberg Fu77 in his ergodic-theoretic proof of Szemerédi's theorem on arithmetic progressions in sets of positive density in $\mathbb{Z}$. Furstenberg proved the following theorem:

1.1. Theorem (Furstenberg). Let $X=\left(X^{0}, \mathcal{B}, \mu, T\right)$ be a measure-preserving system (m.p.s.). Let $A$ be a set of positive measure, and $f=1_{A}$. Then

$$
\liminf _{N \rightarrow \infty} \frac{1}{N} \sum_{n=1}^{N} \int \prod_{j=0}^{k} f\left(T^{j n} x\right) d \mu>0 .
$$

The theorem above ensures that there exists an integer $n$, so that for a set of points $x$ of positive measure the points $x, T^{n} x, \ldots, T^{k n} x$ are in $A$, and corresponds to the existence of an arithmetic progression of length $k+1$ in sets of positive density in $\mathbb{Z}$.

The $L^{2}$ limiting behavior of the averages in (1) is governed by a natural series of "factors" of the measure-preserving system $X$. The factor corresponding to arithmetic progression of length 3 (the case where $k$ is 2), the Kronecker factor, was described in Fu77. The factor corresponding to arithmetic progressions of length 4, an inverse limit of 2-step nilflows, was studied by Conze-Lesigne CL84, CL87, CL88, Furstenberg-Weiss FuW96, and Host-Kra HK01, HK02, and hinted to the nature of the complete series. The complete series of factors was described by Host-Kra HK05, and independently, though somewhat later, by the author. We give an equivalent characterization of the series of factors described in [HK05], and provide a different construction for these factors. Although there are some similarities between the constructions (for example, both start out with the Furstenberg structure theorem [Fu77]), the definition of the factors and the bulk of the construction are significantly different. In particular, we do not use the Gowers uniformity norms G01, which are fundamental in the approach of Host and Kra to this problem (and in the works of Gowers [G01, and Green-Tao GT04] on problems of a similar nature). The averages studied in the paper are of a special

Received by the editors October 18, 2004.

2000 Mathematics Subject Classification. Primary 37Axx.

(C)2006 American Mathematical Society 
kind, but the techniques developed can be used in analyzing other multiple averages (e.g. averages along a polynomial sequence, or cubic averages).

Let $X=\left(X^{0}, \mathcal{B}_{X}, \mu_{X}, T_{X}\right)$ be a probability measure-preserving system; i.e., $\left(X^{0}, \mathcal{B}_{X}, \mu_{X}\right)$ is a measure space, and $T_{X}$ is a measure-preserving transformation. When there is no confusion we will omit the subscript $X$. For a measurable function $f$ we write $T f$ for the function $T f(x)=f(T x)$. By ergodic decomposition it will suffice to study the limit of (10) with the additional hypothesis of ergodicity. The nature of the limit will depend on mixing properties of the system. The maximal degree of mixing relevant is weak mixing; indeed in this case Furstenberg has shown in [Fu77]:

1.2. Theorem (Furstenberg). If $X$ is weak mixing, then

$$
\frac{1}{N} \sum_{n=1}^{N} \prod_{j=1}^{k} f_{j}\left(T^{j n} x\right) \stackrel{L^{2}(X)}{\longrightarrow} \prod_{j=1}^{k} \int f_{j}(x) d \mu .
$$

For a general ergodic system $X$ the averages in equation (11) need not converge to a constant function. Indeed, if the system $X$ is not weakly mixing, there exists a nontrivial eigenfunction $\psi$. If $T \psi(x)=\lambda \psi(x)$, then

$$
T^{n} \psi^{2}(x) T^{2 n} \psi^{-1}(x)=\psi(x)
$$

for all $n$; thus

$$
\frac{1}{N} \sum_{n=1}^{N} T^{n} \psi^{2}(x) T^{2 n} \psi^{-1}(x)=\psi(x) .
$$

By the above equation, the set of limiting functions contains the algebra spanned by eigenfunctions - the Kronecker algebra. The Kronecker algebra determines the "Kronecker factor" $Z$ where $Z^{0}$ is a compact Abelian group, $\mathcal{B}_{Z}$ the (completed) Borel algebra, $\mu_{Z}$ the Haar measure, and the action of $T_{Z}$ is given by translation by an element $\alpha \in Z^{0}$, i.e. $T_{Z} z=z+\alpha$. Let $\pi: X \rightarrow Z$ be the factor map. It is not surprising that an Abelian group factor should come up when studying the relations between $x, T^{n} x, T^{2 n} x$, as the projections of these points on the Abelian group factor $\pi(x), \pi(x)+n \alpha, \pi(x)+2 n \alpha$ form an arithmetic progression: $\pi(x)=$ $2(\pi(x)+n \alpha)-(\pi(x)+2 n \alpha)$. It turns out that this "constraint" imposed by the Kronecker factor is the only "constraint" on the triple $x, T^{n} x, T^{2 n} x$, so that in a manner to be made precise, the Kronecker factor is "characteristic" for the limit of the averages $\frac{1}{N} \sum_{n=1}^{N} f\left(T^{n} x\right) g\left(T^{2 n} x\right)$.

Let $X$ be a measure-preserving system (m.p.s.). Let $Y$ be a homomorphic image; i.e., we have a map $\pi: X^{0} \rightarrow Y^{0}$ with $\pi^{-1} \mathcal{B}_{Y} \subset \mathcal{B}_{X}, \pi \mu_{X}=\mu_{Y}$ and $T_{Y} \pi=\pi T_{X}$. Then $Y$ is a factor of $X, X$ is an extension of $Y$, and abusing the notation we write $\pi: X \rightarrow Y$ for the factor map. A factor of $X$ is determined by a $T_{X}$-invariant subalgebra of $L^{\infty}(X)$. The map $\pi$ induces two natural maps $\pi^{*}: L^{2}(Y) \rightarrow L^{2}(X)$ given by $\pi^{*} f=f \circ \pi$, and $\pi_{*}: L^{2}(X) \rightarrow L^{2}(Y)$ given by $\pi_{*} f=E\left(f \mid \mathcal{B}_{Y}\right)$ (the orthogonal projection of $f$ on $\pi^{*} L^{2}(Y)$ ). The notion of "characteristic factors" was first introduced in a paper by Furstenberg and Weiss [FuW96]. We fix an ergodic invertible m.p.s. $X$.

1.3. Definition. Let $Y$ be a factor of $X$. Let $k$ be a natural number, $\left(a_{1}, \ldots, a_{k}\right)$ be distinct nonzero integers. The system $Y$ is characteristic for $\left(a_{1}, \ldots, a_{k}\right)$ if for 
any $f_{1}, \ldots, f_{k} \in L^{\infty}(X)$,

$$
\frac{1}{N} \sum_{n=1}^{N} \prod_{j=1}^{k} T_{X}^{a_{j} n} f_{j}-\pi^{*} \frac{1}{N} \sum_{n=1}^{N} \prod_{j=1}^{k} T_{Y}^{a_{j} n} \pi_{*} f_{j} \stackrel{L^{2}\left(\mu_{X}\right)}{\longrightarrow} 0 .
$$

The system $Y$ is a $k$-characteristic factor of $X$ if it is characteristic for any $k$-tuple of distinct nonzero integers.

It is in this sense that the Kronecker factor is characteristic for calculating the limit of the averages $\frac{1}{N} \sum_{n=1}^{N} f\left(T^{n} x\right) g\left(T^{2 n} x\right)$. We now define a universal characteristic factor:

1.4. Definition. Let $Y$ be a factor of $X$. The system $Y$ is a $k$-universal characteristic factor (u.c.f.) of $X$ if it is a $k$-characteristic factor of $X$, and a factor of any other $k$-characteristic factor of $X$.

For the averages $\frac{1}{N} \sum_{n=1}^{N} f_{1}\left(T^{n} x\right) f_{2}\left(T^{2 n} x\right) f_{3}\left(T^{3 n} x\right)$, the Kronecker factor does not suffice. Let $\varphi$ be a second-order eigenfunction, i.e., $T \varphi=\psi \varphi$, where $\psi$ is an ordinary eigenfunction, that is, $T \psi=\lambda \psi$. Then one can check that

$$
T^{n} \varphi^{3}(x) T^{2 n} \varphi^{-3}(x) T^{3 n} \varphi(x)=\varphi(x)
$$

for all $n$; thus

$$
\frac{1}{N} \sum_{n=1}^{N} T^{n} \varphi^{3}(x) T^{2 n} \varphi^{-3}(x) T^{3 n} \varphi=\varphi(x) .
$$

Let $Y$ be a factor of $X$ that is characteristic for $(1,2,3)$. Equation (2) implies that the algebra generated by all second-order eigenfunctions is a subset of $L^{\infty}(Y)$. It is natural to conjecture that the algebra generated by the second-order eigenfunctions determines a factor that is characteristic for $(1,2,3)$. Furstenberg and Weiss presented the following counterexample. Let

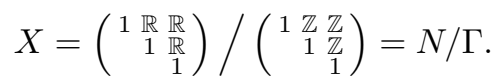

Consider the system $X$ where $X^{0}=N / \Gamma, \mathcal{B}_{X}$ the (completed) Borel algebra, $\mu_{X}$ the unique measure invariant under translations by any element of the group $N$, and $T_{X}$ is given by $T_{X} g \Gamma=a g \Gamma$ for some $a \in N$ acting ergodically. This system has no second-order eigenfunctions, but there are relations between $g \Gamma, a^{n} g \Gamma, a^{2 n} g \Gamma, a^{3 n} g \Gamma$ not coming from the Kronecker factor: in $N / \Gamma, g \Gamma$ is determined by $a^{n} g \Gamma, a^{2 n} g \Gamma$, $a^{3 n} g \Gamma$. This system can be viewed as a circle extension of the Kronecker factor which is a 2-dimensional torus, and the action of $T_{X}$ on $\mathbb{T}^{2} \times S^{1}$ is given by $T_{X}(z, \zeta)=(z+\alpha, \sigma(z) \zeta)$ (the function $\sigma(z)$ is called the extension cocycle). The projection of the points $g \Gamma, a^{n} g \Gamma, a^{2 n} g \Gamma, a^{3 n} g \Gamma$ on the Kronecker factor will form an arithmetic progression, but as $g \Gamma$ is a function of $a^{n} g \Gamma, a^{2 n} g \Gamma, a^{3 n} g \Gamma$ the points $g \Gamma, a^{n} g \Gamma, a^{2 n} g \Gamma, a^{3 n} g \Gamma$ will not be independent on the fibers over the Kronecker factor. This fact translates to a restriction on the extension cocycle $\sigma(z)$ known as the Conze-Lesigne equation. (This equation is analyzed in [CL84, CL87, CL88, Le84, [Le87, [Le93, FuW96, HK01, [HK02, Me90, [R93.) In particular any factor that is characteristic for $(1,2,3)$ will contain functions other than first-order and second-order eigenfunctions.

In general, if $N$ is a $k$-step nilpotent group $\left(N_{k+1}=1\right), \Gamma<N$, then $x \in N / \Gamma$ is determined by $a^{n} x, a^{2 n} x, \ldots, a^{(k+1) n} x$. It is natural to ask whether these are 
the only constraints; i.e., do all the constraints on the points $x, T^{n} x, \ldots, T^{(k+1) n} x$ come from a $k$-step nilpotent factor?

1.5. Definition. A nilsystem consists of a space $X$ on which a nilpotent group $N$ acts transitively preserving a measure $\mu_{X}$, and a transformation $T_{X}$ which acts by translation by a group element $a: T_{X} x=a x$. A special case is when $N$ is a $k$-step nilpotent Lie group, $\Gamma$ a cocompact lattice, $X=N / \Gamma$ (a nilmanifold), and $\mu_{X}$ the unique measure invariant under translation by elements of $N$. We call this a $k$-step nilflow. A $k$-step pro-nilflow is an inverse limit of $k$-step nilflows.

We prove the following theorems:

1.6. Theorem. Let $X$ be an ergodic measure-preserving system. Then there exists a unique $k$-universal characteristic factor of $X$. If $\pi: X \rightarrow Y$ is a factor map, and $W(X), W(Y)$ are $k$-universal characteristic factors of $X, Y$, respectively, then $\pi$ induces a map between $W(X)$ and $W(Y)$.

If we denote by $Y_{k}(X)$ the $k$-u.c.f. of $X$, then one obtains an inverse series of factors $\ldots \rightarrow Y_{k}(X) \rightarrow Y_{k-1}(X) \rightarrow \ldots \rightarrow Y_{1}(X)$.

1.7. Theorem. Let $X$ be an ergodic measure-preserving system, and let $Y_{k}(X)$ be the $k$-universal characteristic factor of $X$. Then $Y_{k}(X)$ has the structure of a $(k-1)$-step nilsystem, more specifically a $(k-1)$-step pro-nilflow.

1.8. Theorem. Let $X$ be an ergodic $(k-1)$-step pro-nilflow. Then $Y_{k}(X)=X$.

The proof of Theorem 1.7 is by induction: assuming that the $k$-u.c.f. is a $(k-1)$ step nilsystem, one reduces the problem of determining the $(k+1)$-u.c.f. to the case where the system $X$ is a circle extension of a $(k-1)$-step nilsystem. If the points $x, T^{n} x, \ldots T^{(k+1) n} x$ are independent on the fibers over the $k$-step nilsystem, then the $k$-step nilsystem would suffice, i.e., would be $k$-characteristic. Otherwise one would get a restriction on the extension cocycle. The main difficulty is using this restriction to construct a nilpotent group acting transitively on $X$.

As a corollary we get the ergodic theorem recently proved by Host and Kra HK05.

1.9. Corollary. Let $X$ be an m.p.s. Let $k$ be a natural number, $a_{1}, \ldots, a_{k} \in \mathbb{Z}$, and $f_{1}, \ldots, f_{k} \in L^{\infty}\left(\mu_{X}\right)$. Then the averages

$$
\frac{1}{N} \sum_{n=1}^{N} \prod_{j=1}^{k} f_{j}\left(T^{a_{j} n} x\right)
$$

converge in $L^{2}\left(\mu_{X}\right)$.

By Theorem 1.7 in order to have $L^{2}\left(\mu_{X}\right)$ convergence of the averages in (3), it is enough to prove an $L^{2}\left(\mu_{X}\right)$ convergence theorem for $k$-step pro-nilflows. Convergence in $L^{2}$ for pro-nilflows follows from convergence for nilflows. For nilflows one has a.e. convergence ([P69], [Sh96], [L05]). An explicit description of the limit is given in [Le89] for the case $k=3$, and in general in [Z05].

\section{UNIVERSAL CHARACTERISTIC FACTORS}

We start by proving the existence of universal characteristic factors (Definitions 1.3 and 1.4). 
2.1. Lemma. Let $X$ be an ergodic m.p.s. Let $Y_{1}, Y_{2}$ be $k$-characteristic factors of $X$. Then there exists a $k$-characteristic factor of $X$, which is a factor of both $Y_{1}, Y_{2}$.

Proof. Let $P, Q$ denote the orthogonal projections onto $L^{2}\left(\mu_{Y_{1}}\right), L^{2}\left(\mu_{Y_{2}}\right)$ (seen as subspaces of $\left.L^{2}\left(\mu_{X}\right)\right)$ respectively, and let $\pi_{i}: X^{0} \rightarrow Y_{i}^{0}$ for $i=1,2$ denote the factor maps. Then $P^{2}=P^{*}=P$ (same for $Q$ ). We show that $(P Q P)^{n}$ converges strongly to a selfadjoint projection operator $W: P$ is a projection; thus $P \leq I$ and

$$
\left\langle(P Q P)^{2} x, x\right\rangle=\langle P Q P x, Q P x\rangle \leq\langle Q P x, Q P x\rangle=\langle P Q P x, x\rangle .
$$

Inductively, the sequence $(P Q P)^{n}$ is a decreasing sequence of operators; thus $\left\langle(P Q P)^{n} x, x\right\rangle$ converges for all $x$. The sequence $(P Q P)^{n} x$ is a Cauchy sequence as

$$
\begin{aligned}
\left\|(P Q P)^{n} x-(P Q P)^{m} x\right\|^{2}= & \left\langle(P Q P)^{2 n} x, x\right\rangle+\left\langle(P Q P)^{2 m} x, x\right\rangle \\
& -2\left\langle(P Q P)^{(n+m)} x, x\right\rangle \rightarrow 0 .
\end{aligned}
$$

Let $W=\lim _{n \rightarrow \infty}(P Q P)^{n}$. Then $W^{2}=W=W^{*}$. If $W x=x$, then $P x=P W x=$ $W x=x$, and

$$
P Q x=P Q P x=P Q P W x=W x=x \Rightarrow\|Q x\|=\|x\| \Rightarrow Q x=x .
$$

It follows that $W\left(L^{2}\left(X^{0}, \mathcal{B}_{X}, \mu_{X}\right)\right)=L^{2}\left(X^{0}, \mathcal{D}, \mu_{X}\right)$ for $\mathcal{D}=\pi_{1}^{-1}\left(\mathcal{B}_{Y_{1}}\right) \cap \pi_{2}^{-1}\left(\mathcal{B}_{Y_{2}}\right)$. We show that $W\left(L^{2}\left(\mu_{X}\right)\right)$ is a $k$-characteristic factor of $X$. For all $m$ :

$$
\begin{aligned}
\lim _{N \rightarrow \infty} & \frac{1}{N} \sum_{n=1}^{N} T^{a_{1} n} f_{1} \ldots T^{a_{k} n} f_{k} \\
& =\lim _{N \rightarrow \infty} \frac{1}{N} \sum_{n=1}^{N} T^{a_{1} n}\left((P Q P)^{m} f_{1}\right) \ldots T^{a_{k} n}\left((P Q P)^{m} f_{k}\right),
\end{aligned}
$$

and the desired conclusion follows by letting $m \rightarrow \infty$.

2.2. Corollary. Let $X$ be an m.p.s. There exists a unique $k$-universal characteristic factor of $X$.

Proof. This follows by Zorn's lemma.

The advantage of looking at all $k$-tuples (rather than focusing on a specific one) is that $k$-u.c.f.s are functorial; any morphism of measure-preserving systems induces a morphism between their $k$-universal characteristic factors, as will be shown in Corollary 2.4. (This may also be true for characteristic factors of a specific scheme.)

2.3. Lemma. Let $V$ be the algebra generated by partial limits of the sequences $\left\{\frac{1}{N} \sum_{n=1}^{N} T^{a_{1} n} f_{1} \ldots T^{a_{k} n} f_{k}\right\}$, where $f_{i} \in L^{\infty}\left(\mu_{X}\right)$, and $a_{0}, \ldots, a_{k} \in \mathbb{Z}$ are distinct nonzero integers. Then $V$ determines the $k$-universal characteristic factor of $X$.

Proof. Let $W(X)$ be the $k$-u.c.f. Obviously $V \subset L^{\infty}\left(\mu_{W(X)}\right)$. We show that the factor determined by $V$ is a $k$-characteristic factor of $X$. Let $f_{1} \perp V$. Then for any 


$$
\begin{aligned}
g \in L^{\infty}\left(\mu_{X}\right), & \\
\left\langle g, \frac{1}{N} \sum_{n=1}^{N} T^{a_{1} n} f_{1} \ldots T^{a_{k} n} f_{k}\right\rangle & =\frac{1}{N} \sum_{n=1}^{N} \int g T^{a_{1} n} f_{1} T^{a_{2} n} f_{2} \ldots T^{a_{k} n} f_{k} d \mu \\
& =\frac{1}{N} \sum_{n=1}^{N} \int f_{1} T^{-a_{1} n} g \ldots T^{\left(a_{k}-a_{1}\right) n} f_{k} d \mu \\
& \stackrel{N \rightarrow \infty}{\longrightarrow} 0 .
\end{aligned}
$$

2.4. Corollary. If $\pi: X \rightarrow Y$ is a factor map, and $W(X), W(Y)$ are k-u.c.f. for $X, Y$, respectively, then $\pi$ induces a map between $W(X)$ and $W(Y)$.

2.5. Universal characteristic factors for $k=1,2$. Let $X$ be an ergodic m.p.s. If the system is totally ergodic, i.e., if every nonzero power of $T_{X}$ is ergodic, then the ergodic theorem applies to the average in question, and the limit is a constant, so that the characteristic factor can be taken as a trivial system. Otherwise one needs to take into account the algebra generated by functions that are invariant under $T_{X}^{m}$ for some $m$. The existence of such functions corresponds to having as a factor the cyclic group $\mathbb{Z} / m \mathbb{Z}$ with the action $x \rightarrow x+1(\bmod m)$. All such factors can be taken into account by a "pro-cyclic" group factor, the group being the inverse limit of finite cyclic groups. This group with the transformation $x \rightarrow x+1$ will then represent the 1-u.c.f. of $X$. The 2-u.c.f. of $X$ coincides with the first block in Furstenberg's structure theorem (see [Fu77]) and is referred to as the Kronecker factor. The system $Z$ is a Kronecker system (or an almost periodic system) if $Z^{0}$ is a compact abelian group (a 1-step nilpotent group), $\mathcal{B}_{Z}$ is the (completed) Borel algebra, $\mu_{Z}$ is the Haar measure, and the action of $T_{Z}$ is given by $T_{Z} z=z+\alpha$ for some $\alpha \in Z^{0}$. The Kronecker factor is the maximal almost periodic factor. Equivalently, $Z$ is the Kronecker factor of $X$ if the eigenfunctions of $T_{X}$ span $L^{2}\left(\mu_{Z}\right)$ (thought of as a subspace of $L^{2}\left(\mu_{X}\right)$ ).

2.6. Remark. If the system $X$ is weak mixing, i.e. has no nontrivial eigenfunctions, then the Kronecker factor is trivial (and $Y_{k}(X)$ is trivial for all $k$ ).

2.7. Isometric extensions The notion of characteristic factors was motivated by Furstenberg's structure theorem Fu77. Furstenberg's idea was to relativize the notion of weak mixing to a weak mixing extension and to define the complementary notion of a compact extension (or isometric extension). Let $X$ be an ergodic m.p.s., and let $Y$ be a factor. Consider the ring $L^{\infty}\left(\mu_{Y}\right)$ as a subring of functions on $X$. A subspace $V \subset L^{2}\left(\mu_{X}\right)$ is a finite rank module over $L^{\infty}\left(\mu_{Y}\right)$ if there exist finitely many functions $\varphi_{1}, \ldots, \varphi_{k}$, such that any function $f \in V$ can be expressed as $f=\sum_{i=1}^{k} a_{i}(y) \varphi_{i}(x)$. We say that $X$ is an isometric extension of $Y$ if $L^{2}\left(\mu_{X}\right)$ is spanned by finite rank $T_{X}$ invariant modules over $L^{\infty}\left(\mu_{Y}\right)$. It can be shown that in this case $X$ is isomorphic to a skew product $X^{\prime}$ where $X^{\prime 0}=Y^{0} \times M$, where $M=G / H$ is a homogeneous compact metric space, $\mu_{X^{\prime}}=\mu_{Y} \times m_{M}$, where $m_{M}$ is the unique probability measure invariant under the transitive group of isometries $G$, and the action of $T_{X^{\prime}}$ is given by $T_{X^{\prime}}(y, m)=\left(T_{Y} y, \rho(y) m\right)$, where $\rho: Y^{0} \rightarrow G$. We denote $T_{X^{\prime}}$ by $T_{Y, \rho}$, or if there is no confusion, just $T_{\rho}$. For example, a Kronecker system is an isometric extension of a point. Define the $n$th 
iteration of $\rho, \rho^{(n)}: Y^{0} \rightarrow G$ by $T_{\rho}^{n}(y, m)=\left(T^{n} y, \rho^{(n)}(y) m\right)$; then $\rho^{(n)}$ satisfies a 1-cocycle equation for the action of $\mathbb{Z}$ on functions from $Y$ to $G$ :

$$
\rho^{(n+m)}(y)=\rho^{(n)}\left(T^{m} y\right) \rho^{(m)}(y) .
$$

Since $\rho^{(n)}(y)$ is determined by $\rho^{(1)}(y)$ we shall focus on $\rho(y)=\rho^{(1)}(y)$ and refer to it as the extension cocycle (or just cocycle). Abusing the notation we denote the system $X^{\prime}$ by $Y \times{ }_{\rho} G / H$. For more details see [Fu77] or [Zi76].

2.8. Let $X_{1}, X_{2}$ be m.p.s. and let $Y$ be a common factor with $\pi_{i}: X_{i}^{0} \rightarrow Y^{0}$ for $i=1,2$ the factor maps. Let $\mu_{X_{i}, y}$ represent the disintegration of $\mu_{X_{i}}$ with respect to $Y$. Let $\mu_{X_{1}} \times_{Y} \mu_{X_{2}}$ denote the measure defined by

$$
\mu_{X_{1}} \times_{Y} \mu_{X_{2}}(A)=\int \mu_{X_{1}, y} \times_{Y} \mu_{X_{2}, y}(A) d \mu_{Y}
$$

for $A \in \mathcal{B}_{X_{1}} \times \mathcal{B}_{X_{2}}$. The system

$$
\left(X_{1}^{0} \times X_{2}^{0}, \mathcal{B}_{X_{1}} \times \mathcal{B}_{X_{2}}, \mu_{X_{1}} \times_{Y} \mu_{X_{2}}, T_{X_{1}} \times T_{X_{2}}\right)
$$

is called the relative product of $X_{1}$ and $X_{2}$ with respect to $Y$ and is denoted $X_{1} \times_{Y} X_{2}$.

2.9. Let $X$ be an ergodic m.p.s., $Y$ a factor and $\pi: X \rightarrow Y$ the factor map. Consider the subspace of $L^{2}\left(\mu_{X}\right)$ spanned by all finite rank $T_{X}$-invariant modules over $\pi^{*} L^{\infty}\left(\mu_{Y}\right)$. This subspace will be defined by some factor $\hat{Y}$ between $X$ and $Y$. The system $\hat{Y}$ is called the maximal isometric extension of $Y$ in $X$. For some $l \in \mathbb{N}$, let $X^{\prime}=\left(X^{0}, \mathcal{B}_{X}, \mu_{X}, T_{X}^{l}\right)$, and let $Y^{\prime}=\left(Y^{0}, \mathcal{B}_{Y}, \mu_{Y}, T_{Y}^{l}\right)$. Then the maximal isometric extension of $Y^{\prime}$ in $X^{\prime}$ is $\hat{Y}^{\prime}=\left(\hat{Y}^{0}, \mathcal{B}_{\hat{Y}}, \mu_{\hat{Y}}, T_{\hat{Y}}^{l}\right)$.

2.10. Let $X_{i}, i=1, \ldots, k$, be measure-preserving systems, and let $Y_{i}$ be corresponding factors, and $\pi_{i}: X_{i}^{0} \rightarrow Y_{i}^{0}$ the factor maps. A measure $\nu$ on $\Pi Y_{i}^{0}$ defines a joining of the measures on $Y_{i}$ if it is invariant under $T_{Y_{1}} \times \ldots \times T_{Y_{K}}$ and maps onto $\nu_{Y_{i}}$ under the natural map $\Pi Y_{i} \rightarrow Y_{j}$. Let $\nu$ be a joining of the measures on $Y_{i}$, and let $\mu_{X_{i}, y_{i}}$ represent the disintegration of $\mu_{X_{i}}$ with respect to $Y_{i}$.

Let $\mu$ be a measure on $\Pi X_{i}^{0}$ defined by

$$
\mu=\int \mu_{X_{1}, y_{1}} \times \ldots \times \mu_{X_{k}, y_{k}} d \nu\left(y_{1}, \ldots, y_{k}\right) .
$$

Then $\mu$ is called the conditional product measure with respect to $\nu$.

The following is shown in Fu77, Theorem 9.5]:

2.11. Theorem (Furstenberg). Let $X_{i}, Y_{i}, \nu, \mu$ be as in 2.10. Assume each $X_{i}$ has finitely many ergodic components. Let $\hat{Y}_{i}$ be the maximal isometric extension of $Y_{i}$ in $X_{i}, \hat{\pi}_{i}: X_{i} \rightarrow \hat{Y}_{i}$ the projection. Then if $F \in L^{2}(\mu)$ is invariant under $T_{X_{1}} \times \ldots \times T_{X_{k}}$, then there exists a function $\Phi \in L^{2}\left(\Pi \hat{Y}_{i}, \Pi \hat{\nu}_{i}\right)$, so that

$$
F\left(x_{1}, \ldots, x_{k}\right)=\Phi\left(\hat{\pi}_{1}\left(x_{1}\right), \ldots, \hat{\pi}_{k}\left(x_{k}\right)\right) .
$$

2.12. Group extensions. A special case of an isometric extension $X \rightarrow Y$ is when the homogeneous space $M$ from 2.7 is equal to $G$, i.e. $X=Y \times{ }_{\rho} G$, where $G$ is a compact group. In this case we say that $X$ is a group extension of $Y$.

2.13. Lemma. Suppose $X$ is an ergodic isometric extension of $Y$ so that we can express $X=Y \times{ }_{\rho} G / H$. Using the function $\rho$, we can define a group extension $Y \times{ }_{\rho} G$. Then $G$ and $H$ can be chosen so that the extension $Y \times{ }_{\rho} G$ is an ergodic group extension. 
Proof. [FuW96, Lemma 7.2].

2.14. Lemma. Let $X=Y \times{ }_{\rho} G$ be an ergodic group extension of $Y$, and let $W$ be an intermediate factor between $X$ and $Y$. Then $X$ is a group extension of $W$.

Proof. [FuW96, Lemma 7.3].

2.15. Let $Y$ be an ergodic m.p.s., $G$ a compact metrizable group. Let $Y \times{ }_{\rho} G$ be a group extension. We can parameterize $Y^{0} \times G$, replacing $(y, g)$ with $F(y, g)=$ $(y, f(y) g)$ for some measurable function $f: Y^{0} \rightarrow G$. Let $\rho^{\prime}(y):=f(T y) \rho(y) f(y)^{-1}$. Then the systems $Y \times{ }_{\rho} G, Y \times \times_{\rho^{\prime}} G$ are isomorphic, and $\rho, \rho^{\prime}$ are called equivalent cocycles or cohomologous cocycles. If $\rho$ is equivalent to the identity cocycle, then $\rho$ is a $Y$-coboundary (or just coboundary when there is no confusion). If $\rho$ is equivalent to a constant cocycle, then $\rho$ is a $Y$-quasi-coboundary (or quasi-coboundary).

2.16. If $\rho$ takes values in a closed subgroup $H$ of $G$, the extension $Y \times{ }_{\rho} G$ will not be ergodic (any function on $H \backslash G$ will be invariant). By the foregoing discussion if $\rho$ is equivalent to a cocycle taking values in a closed subgroup $H$, then the extension $Y \times_{\rho} G$ will not be ergodic.

2.17. Theorem (Mackey). Let $\rho: Y^{0} \rightarrow G$ be a measurable cocycle. There exists a closed subgroup $M<G$, unique up to conjugacy, so that:

(1) $\rho$ is equivalent to a cocycle $\rho^{\prime}$ taking values in $M$, i.e., $\rho^{\prime}(y)=f(T y) \rho(y) f(y)^{-1} \in M$.

(2) Any ergodic $T_{\rho^{\prime}}$-invariant measure on $Y^{0} \times G$, extending $\mu_{Y}$, has the form $\mu_{Y} \times m_{M \gamma}$ for some coset $M \gamma$, and the ergodic $T_{\rho}$-invariant measures are obtained by applying $F^{-1}$ (defined in 2.15) to the ergodic $T_{\rho^{\prime}}$-invariant measures. The group $M$ is called the Mackey group of the extension $Y \times{ }_{\rho} G$.

2.18. Lemma. For $i=1,2$, let $Y_{i}$ be an ergodic m.p.s., let $X_{i}=Y_{i} \times_{\rho_{i}} G$ be group extensions, and let $M_{i}$ be the associated Mackey groups. Let $\pi_{i}$ be the projection $\pi_{i}: X_{i} \rightarrow Y_{i}$. Let $S: X_{1} \rightarrow X_{2}$ be an isomorphism inducing an isomorphism of $Y_{1}$ to $Y_{2}$, and suppose $S \pi_{1}=\pi_{2} S$. Then $M_{1}$ and $M_{2}$ are conjugate.

Proof. The transformation $S$ maps the ergodic components of the group extension $Y_{1} \times{ }_{\rho_{1}} G$ onto those of $Y_{2} \times \rho_{2} G$. Each ergodic component is determined by a right coset $M_{i} \gamma_{i}$ for $\gamma_{i} \in G$; thus $S$ induces a map from $\varphi: M_{1} \backslash G \rightarrow M_{2} \backslash G$ that commutes with the action of $G$ from the right. Thus $\varphi$ is a $G$-isomorphism, and therefore $M_{1}$ and $M_{2}$ are conjugate.

\section{Abelian extensions}

3.1. Notation. We use additive notation for abelian groups with the exception of the group $S^{1}=\{\zeta \in \mathbb{C}:|\zeta|=1\}$, which will play a special role in the future. In particular, if $\rho, \rho^{\prime}$ are equivalent cocycles (defined in the foregoing section) taking values in an abelian group $G$, then there exists a function $f: Y^{0} \rightarrow G$ such that

$$
\rho(y)=f(T y)+\rho^{\prime}(y)-f(y) .
$$

3.2. Let $G$ be a compact abelian group. Then $Y \times{ }_{\rho} G$ is an abelian extension. In this case the Mackey group defined in the foregoing section $M$ is unique. Let

$$
M^{\perp}=\{\chi \in \hat{G}: \chi(g)=1 \text { for all } g \in M\}
$$


be the annihilator of $M$. If $\rho$ is equivalent to a cocycle taking values in $M$, then $\chi \circ \rho$ is a coboundary for all $\chi \in \hat{M}$ and

$$
M^{\perp}=\{\chi \in \hat{G}: \chi \circ \rho \text { is a coboundary }\} .
$$

3.3. Proposition. Let $Y \times{ }_{\rho} G$ be an abelian extension, and let $M$ be the Mackey group of this extension. Let $f \in L^{2}\left(\mu_{Y} \times m_{G}\right)$ be s.t. for all $\chi \in M^{\perp}$,

$$
\int f(y, g) \chi(g) d m_{G}(g)=0
$$

for a.e. $y \in Y$. Then $f$ is orthogonal to the space of $T_{\rho}$-invariant functions.

Proof. [FuW96, Lemma 9.2].

3.4. Notation. Denote $U_{d}=d$-dimensional unitary matrices, $C\left(U_{d}\right)$ the center of $U_{d}$ (scalar matrices), and $P: U_{d} \rightarrow \mathbb{P} U_{d}=U_{d} / C\left(U_{d}\right)$ the natural projection. For $U, V \in U_{d}$ denote by $[U, V]$ the commutator of $U, V$; i.e., $[U, V]=U V U^{-1} V^{-1}$.

We need the following lemma:

3.5. Lemma. Let $H$ be a compact abelian connected group, and $A: H \rightarrow U_{d} a$ measurable function. If $P \circ A$ is a homomorphism, then $A(H)$ is a commuting set of matrices.

Proof. Let $g, h \in H$. Suppose $[A(h), A(g)]=\delta I$. If $v$ is an eigenvector of $A(h)$ with eigenvalue $\gamma$, then

$$
A(h) A(g) v=\delta A(g) A(h) v=\gamma \delta A(g) v ;
$$

thus $A(g) v$ is an eigenvector of $A(h)$ with eigenvalue $\gamma \delta$. This implies that $A(g)^{k} v$ is an eigenvector of $A(h)$ with eigenvalue $\gamma \delta^{k}$; thus $\delta$ is a root of unity of order $\leq d$. Denote by $C_{d !}$ the group of order $d$ ! roots of unity. Then the commutator set

$$
\{[A(h), A(g)]\}_{h, g \in H} \subset C_{d !} I .
$$

Fix $g$. The function $h \rightarrow[A(h), A(g)]$ is a measurable homomorphism to $C_{d !} I$,

$$
\left[A\left(h_{1}+h_{2}\right), A(g)\right]=\left[c A\left(h_{1}\right) A\left(h_{2}\right), A(g)\right]=\left[A\left(h_{1}\right), A(g)\right]\left[A\left(h_{2}\right), A(g)\right],
$$

therefore continuous, and as $H$ is connected, it is trivial.

3.6. Theorem. Let $Y$ be an ergodic m.p.s., and let $W=Y \times_{\rho} H$ be an ergodic extension by a connected abelian group. Let $F: Y^{0} \times H \times H \rightarrow S^{1}$ be a measurable function. Let $\sigma_{1}\left(y, h_{1}\right), \sigma_{2}\left(y, h_{2}\right): Y^{0} \times H \rightarrow S^{1}$ be measurable functions. Suppose

$$
\sigma_{1}\left(y, h_{1}\right) \sigma_{2}\left(y, h_{2}\right)=\frac{F\left(T y, h_{1}+\rho(y), h_{2}+\rho(y)\right)}{F\left(y, h_{1}, h_{2}\right)} .
$$

Then for $i=1,2$ there exist measurable functions $g_{i}, G_{i}: Y^{0} \rightarrow S^{1}$ such that

$$
\sigma_{i}(y, h)=g_{i}(y) \frac{G_{i}\left(T_{W}(y, h)\right)}{G_{i}(y, h)} .
$$

Proof. We construct the following systems: for $i=1,2$, let $X_{i}=W \times_{\sigma_{i}} S^{1}$ and $X=X_{1} \times_{Y} X_{2}$. Let $\mu_{X}$ be defined as the conditional product measure relative to the diagonal measure on $Y^{0} \times Y^{0}$. The function

$$
\tilde{F}\left(y, h_{1}, h_{2}, \zeta_{1}, \zeta_{2}\right)=F\left(y, h_{1}, h_{2}\right) \zeta_{1}^{-1} \zeta_{2}^{-1}
$$


is invariant under $T_{X}$, and therefore by Theorem 2.11 it is measurable with respect to $\hat{Y}_{1} \times \hat{Y}_{2}$, where $\hat{Y}_{i}$ is the maximal isometric extension of $Y$ in $X_{i}$ for $i=1,2$. Isometric extensions are spanned by finite rank modules (see 2.7). Thus

$$
\tilde{F}\left(y, h_{1}, h_{2}, \zeta_{1}, \zeta_{2}\right)=\sum\left\langle\overrightarrow{\psi_{j}^{1}}\left(y, h_{1}, \zeta_{1}\right), \overrightarrow{\psi_{j}^{2}}\left(y, h_{2}, \zeta_{2}\right)\right\rangle
$$

where

$$
\begin{aligned}
T_{X_{1}} \overrightarrow{\psi_{j}^{1}}\left(y, h_{1}, \zeta_{1}\right) & =U_{j}^{1}(y) \overrightarrow{\psi_{j}^{1}}\left(y, h_{1}, \zeta_{1}\right), \\
T_{X_{2}} \overrightarrow{\psi_{j}^{2}}\left(y, h_{2}, \zeta_{i}\right) & =U_{j}^{2}(y) \overrightarrow{\psi_{j}^{2}}\left(y, h_{2}, \zeta_{2}\right),
\end{aligned}
$$

and $U_{j}^{1}(y), U_{j}^{2}(y)$ are $d_{j} \times d_{j}$ unitary matrices. Substituting the Fourier expansions:

$$
\begin{aligned}
& \overrightarrow{\psi_{j}^{1}}\left(y, h_{1}, \zeta_{1}\right)=\sum \overrightarrow{\psi_{j, k}^{1}}\left(y, h_{1}\right) \zeta_{1}^{k}, \\
& \overrightarrow{\psi_{j}^{2}}\left(y, h_{2}, \zeta_{2}\right)=\sum \overrightarrow{\psi_{j, k}^{2}}\left(y, h_{2}\right) \zeta_{2}^{k}
\end{aligned}
$$

in equation (4) we get that for $k=-1$ there exists $j$ such that $\psi_{j,-1}^{1} \neq 0$. Apply $T_{X_{1}}$ to get

$$
\sigma_{1}^{-1}\left(y, h_{1}\right) \psi_{j,-1}^{\overrightarrow{1}}\left(T_{W}\left(y, h_{1}\right)\right)=U_{j}^{1}(y) \psi_{j,-1}^{\overrightarrow{1}}\left(y, h_{1}\right) .
$$

For simplicity we drop the indices:

$$
\sigma^{-1}(y, h) \vec{\psi}\left(T_{W}(y, h)\right)=U(y) \vec{\psi}(y, h) .
$$

For each $y$ consider the distribution of $\vec{\psi}(y, h)$ in the fiber over $y$, and look at the vector space spanned by the support of this distribution. Call this $V_{y}$, so that $V_{y} \subset \mathbb{C}^{d}$, and $V_{T y}=U(y) V_{y}$. Since $U(y)$ is unitary, $\operatorname{dim} V_{T y}=\operatorname{dim} V_{y}$; thus by ergodicity, $\operatorname{dim} V_{y}=\hat{d}$ for a.s. $y$. For each $y$ choose a basis for $\mathbb{C}^{d}$ s.t. $V_{y}$ is spanned by the first $\hat{d}$ elements. As the transformation matrix is a function of $y$, we may assume $d=\hat{d}$.

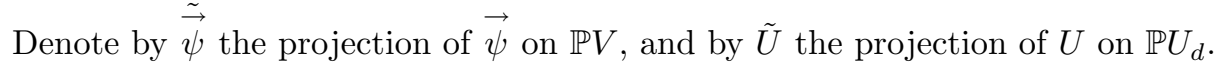
Thus:

$$
\tilde{\vec{\psi}}\left(T_{W}(y, h)\right)=\tilde{U}(y) \tilde{\vec{\psi}}(y, h) .
$$

Consider the group extension $W \times_{\tilde{U}} \mathbb{P} U_{d}$. Then

$$
\tilde{\vec{\psi}}\left(T_{W}^{n}(y, h)\right)=\tilde{U}^{(n)}(y) \stackrel{\overrightarrow{\vec{\psi}}}{(y, h)} .
$$

For fixed $y,\{\tilde{\vec{\psi}}(y, h)\}_{h \in H}$ spans the space, so whenever $T_{W}^{n}(y, h)=\left(T^{n} y, h+\right.$ $\left.\rho^{(n)}(y)\right)$ is close to $(y, h)$ (by ergodicity this happens for a generic $y$, and is independent of $h), \tilde{U}^{(n)}(y)$ is close to the identity. This implies that the foregoing group extension is not ergodic, and furthermore, the Mackey group is trivial. Thus for some projective unitary matrix function $\tilde{M}$ :

$$
\tilde{M}\left(T_{W}(y, h)\right)=\tilde{U}(y) \tilde{M}(y, h) .
$$

Also for any $h^{\prime}$,

$$
\tilde{M}\left(T_{W}\left(y, h+h^{\prime}\right)\right)=\tilde{U}(y) \tilde{M}\left(y, h+h^{\prime}\right)
$$

Thus

$$
\tilde{M}^{-1}\left(T_{W}\left(y, h+h^{\prime}\right)\right) \tilde{M}\left(T_{W}(y, h)\right)=\tilde{M}^{-1}\left(y, h+h^{\prime}\right) \tilde{M}(y, h) .
$$


By ergodicity

$$
\tilde{M}^{-1}\left(y, h+h^{\prime}\right) \tilde{M}(y, h)=\tilde{A}\left(h^{\prime}\right),
$$

for all $h^{\prime}$, a.e. $(y, h)$. By Fubini's theorem there exists $h_{0}$ such that

$$
\tilde{M}(y, h)=\tilde{M}\left(y, h_{0}\right) \tilde{A}^{-1}\left(h-h_{0}\right),
$$

for a.e. $(y, h)$. The function $\tilde{A}\left(h^{\prime}\right)$ is a homomorphism of $H$ :

$$
\begin{aligned}
\tilde{A}\left(h^{\prime}+h^{\prime \prime}\right) & =\tilde{M}^{-1}\left(y, h+h^{\prime}+h^{\prime \prime}\right) \tilde{M}(y, h) \\
& =\tilde{M}^{-1}\left(y, h+h^{\prime}+h^{\prime \prime}\right) \tilde{M}\left(y, h+h^{\prime}\right) \tilde{M}^{-1}\left(y, h+h^{\prime}\right) \tilde{M}(y, h) \\
& =\tilde{A}\left(h^{\prime \prime}\right) \tilde{A}\left(h^{\prime}\right) .
\end{aligned}
$$

Recall $P: U_{d} \rightarrow \mathbb{P} U_{d}$ is the natural projection. We can find a measurable function $A: H \rightarrow U_{d}$ so that $P \circ A=\tilde{A}$ and

$$
A(H) \subset P^{-1} \tilde{A}(H) .
$$

Then by Lemma 3.5. $A(H)$ is a commuting set. Substituting equation (7) in equation (6) we get

$$
\begin{aligned}
\tilde{M}\left(T y, h_{0}\right) \tilde{A}^{-1}\left(h+\rho(y)-h_{0}\right) & =\tilde{M}(T y, h+\rho(y)) \\
& =\tilde{U}(y) \tilde{M}(y, h) \\
& =\tilde{U}(y) \tilde{M}\left(y, h_{0}\right) \tilde{A}^{-1}\left(h-h_{0}\right) .
\end{aligned}
$$

Thus

$$
\tilde{U}(y)=\tilde{M}\left(T y, h_{0}\right) \tilde{A}^{-1}(\rho(y)) \tilde{M}^{-1}\left(y, h_{0}\right)
$$

or

$$
U(y)=M\left(T y, h_{0}\right) A(-\rho(y)) M^{-1}\left(y, h_{0}\right) d(y),
$$

where $d(y)$ is a scalar matrix. As $A(H)$ is a commuting set, it is simultaneously diagonalizable:

$$
A(h)=N^{-1} D(h) N .
$$

Therefore

$$
U(y)=M\left(T y, h_{0}\right) N^{-1} D(-\rho(y)) N M^{-1}\left(y, h_{0}\right) d(y) .
$$

Denote $M^{\prime}(y)=M\left(y, h_{0}\right)$. Substitute $U(y)$ in equation (5):

$$
\sigma^{-1}(y, h) N M^{\prime-1}(T y) \vec{\psi}(T y, h+\rho(y))=D(-\rho(y)) d(y) N M^{\prime-1}(y) \vec{\psi}(y, h) .
$$

Now each coordinate gives us the desired result.

3.7. Remark. If $H$ in Theorem 3.6 is not necessarily connected, but the cocycle $\rho$ is cohomologous to a constant: $\rho(y)=c \frac{f(T y)}{f(y)}$, then we do not need to use Lemma 3.5. and the result holds as for some scalar matrix $d(y): Y^{0} \rightarrow S^{1}$,

$$
A(\rho(y))=A\left(c f(T y) f^{-1}(y)\right)=A(f(T y)) A(c) A^{-1}(f(y)) d(y) .
$$

Now diagonalize $A(c): A(c)=U D U^{-1}$ and substitute in equation (8). 
3.8. Theorem. Let $Y=\left(Y^{0}, \mathcal{B}_{Y}, \mu_{Y}, T_{Y}\right)$ be an ergodic m.p.s. For $i=1, \ldots, k$ let $Y_{i}=\left(Y^{0}, \mathcal{B}_{Y}, \mu_{Y}, T_{Y}^{i}\right)$. Let $W=Y \times_{\rho} H$ be an ergodic group extension, where $H$ is a connected abelian group, and let $W_{i}=Y_{i} \times_{\rho^{(i)}} H$ (notice that $T_{W_{i}}=T_{W}^{i}$ ). Let $\nu$ be a joining of the measures on $Y_{i}$, and let $\mu$ be a measure on $\Pi W_{i}^{0}$ that is the conditional product measure with respect to $\nu$. Let $F: \Pi W_{i}^{0} \rightarrow S^{1}$ be a $\mu$ measurable function. For $i=1, \ldots, k$, let $\sigma_{i}: W^{0} \rightarrow S^{1}$ be measurable functions, and denote by $\pi: W^{0} \rightarrow Y^{0}$ the projection. Suppose $\mu$ a.e.

$$
\prod_{i=1}^{k} \sigma_{i}^{(i)}\left(w_{i}\right)=\frac{F\left(T_{W_{1}} w_{1}, \ldots, T_{W_{k}} w_{k}\right)}{F\left(w_{1}, \ldots, w_{k}\right)} .
$$

Then there exist measurable functions $g_{i}, G_{i}: Y^{0} \rightarrow S^{1}$ such that

$$
\sigma_{i}^{(i)}(w)=g_{i}(\pi(w)) \frac{G_{i}\left(T_{W_{i}}(w)\right)}{G_{i}(w)}
$$

Proof. The proof is similar to the proof of Theorem 3.6. For $i=1, \ldots, k$ let $X_{i}=W_{i} \times_{\sigma_{i}} S^{1}$. Let $X$ be the system with $X^{0}=\prod X_{i}^{0}, \mu_{X}$ the conditional product measure with respect to $\nu$, and $T_{X}=T_{X_{1}} \times \ldots \times T_{X_{k}}$. The function

$$
\tilde{F}\left(w_{1}, \zeta_{1}, \ldots, w_{k}, \zeta_{k}\right)=F\left(w_{1}, \ldots, w_{k}\right) \zeta_{1}^{-1} \ldots \zeta_{k}^{-1}
$$

is invariant under $T_{X}$. Proceeding as in Theorem 3.6 we find that equation (10) holds on the ergodic components of $T_{W}^{i}$. As $T_{W}$ is ergodic, $T_{W}^{i}$ has finitely many ergodic components. Let $Y_{l}$ be an ergodic component of $T_{Y}^{i}$. The ergodic components of $T_{W}^{i}$ which project onto $Y_{l}$ are determined by the Mackey group $M_{l}$ which is a closed subgroup of $H$. As $T_{W}^{i}$ has finitely many ergodic components, $M_{l}$ is of finite index in $H$, but $H$ is connected, therefore has no closed subgroups of finite index. Therefore the ergodic components of $T_{W}^{i}$ are of the form $Y_{l} \times H$.

3.9. Corollary. Let $Y$ be an ergodic m.p.s., $X=Y \times{ }_{\rho} H$ an ergodic abelian extension where either $H$ is connected or the cocycle $\rho$ is cohomologous to a constant. Suppose there exists a measurable family of measurable functions $\left\{f_{u}\right\}_{u \in H}$, $f_{u}: Y^{0} \times H \rightarrow S^{1}$ such that

$$
\frac{\sigma(y, h+u)}{\sigma(y, h)}=\frac{f_{u}\left(T_{X}(y, h)\right)}{f_{u}(y, h)} .
$$

Then there exist measurable functions $g: Y^{0} \rightarrow S^{1}$ and $F: Y^{0} \times H \rightarrow S^{1}$ such that

$$
\sigma(y, h)=g(y) \frac{G\left(T_{X}(y, h)\right)}{G(y, h)}
$$

Proof. Make the coordinate change: $h_{1}=h+u ; h_{2}=h$. Then

$$
f_{u}(y, h)=f(y, u, h)=f^{\prime}(y, h+u, h)=f^{\prime}\left(y, h_{1}, h_{2}\right)
$$

and

$$
f_{u}(T y, h+\rho(y))=f^{\prime}\left(T y, h_{1}+\rho(y), h_{2}+\rho(y)\right) .
$$

Now apply Theorem 3.6 ,

3.10. Lemma. Let $Y=Z \times{ }_{\rho} H$ be an ergodic abelian extension of $Z$, and $F$ : $Z^{0} \times H \rightarrow S^{1}, g: Z^{0} \rightarrow S^{1}$ measurable functions such that

$$
g(z)=\frac{T_{Y} F(z, h)}{F(z, h)} .
$$


Then there exists $\chi \in \hat{H}$, and $k: Z^{0} \rightarrow S^{1}$ such that

$$
F(z, h)=k(z) \chi(h) \text {. }
$$

Proof. Take the Fourier expansion of $F$ :

$$
F(z, h)=\sum k_{i}(z) \chi_{i}(h) .
$$

Then for all $i$,

$$
k_{i}\left(T_{Z} z\right) \chi_{i}(h) \chi_{i}(\rho(z))=g(z) k_{i}(z) \chi_{i}(h) .
$$

Ergodicity of $T_{Z}$ implies that $\left|k_{i}(z)\right|$ is constant a.e. The fact that $|F|=1$ implies that there exist an $i$ for which $\left|k_{i}(z)\right| \neq 0$. If there are two such indices $i, j$, then

$$
\frac{\chi_{i}}{\chi_{j}}(\rho(z))
$$

is a coboundary. As $T_{Y}$ is ergodic, $\chi_{i} / \chi_{j}=1$ (otherwise the Mackey group of the extension $Z \times{ }_{\rho} H$ is not $H$ ).

3.11. Notation. Let $\left(X_{1}^{0}, \mathcal{B}_{1}\right),\left(X_{2}^{0}, \mathcal{B}_{2}\right)$ be measure spaces. Denote

$$
B\left(X_{1}^{0}, X_{2}^{0}\right)=\left\{f: X_{1}^{0} \rightarrow X_{2}^{0}, f \text { measurable }\right\} .
$$

3.12. Lemma. Let $Y=Z \times{ }_{\rho} H$ be an ergodic abelian extension of $Z,(X, \mu)$ a measure space, and let $x \rightarrow f_{x}(y)$ be a Borel measurable function from $X$ to $B\left(Y^{0}, S^{1}\right)$. Suppose for all $x \in X$ there are functions $g_{x}(z), F_{x}(y) \in B\left(Y^{0}, S^{1}\right)$ such that

$$
f_{x}(y)=g_{x}(z) \frac{T_{Y} F_{x}(y)}{F_{x}(y)} .
$$

Then there is a $\mu$ measurable choice of $g_{x}(z), F_{x}(y)$.

Proof. Endowed with the $L^{2}$ topology, $B\left(Y^{0}, S^{1}\right)$ is a Polish group. Let $B\left(Z^{0}, S^{1}\right)$ be the closed subgroup of $B\left(Y^{0}, S^{1}\right)$ of functions that depend only on the $z$ coordinate, and let $f \rightarrow \bar{f}$ be the natural projection onto $\bar{B}=B\left(Y^{0}, S^{1}\right) / B\left(Z^{0}, S^{1}\right)$, with the induced topology. By a theorem of Dixmier (BK96, Theorem 1.2.4) there is a measurable section $s: \bar{B} \rightarrow B$. Equation (12) implies that

$$
\bar{f}_{x}(y)=\frac{T_{Y} \bar{F}_{x}(y)}{\bar{F}_{x}(y)} .
$$

Define $\varphi: \bar{B} \rightarrow \bar{B}$ by $\varphi(\bar{f})=T_{Y} \bar{f} / \bar{f}$. If $\varphi(\bar{f})=\varphi(\bar{g})$, then for some function $h(z)$,

$$
\frac{T_{Y} \frac{f}{g}(y)}{\frac{f}{g}(y)}=h(z) .
$$

By 3.10 this implies that up to multiplication by a function of $z, \frac{f}{g}$ belongs to a countable set; thus $\varphi$ is countable to one. By Lusin [Lu30 $\varphi(\bar{B})$ is a measurable set and there is a measurable function $\psi: \varphi(\bar{B}) \rightarrow \bar{B}$ s.t.

Now if

$$
\varphi \circ \psi=\left.I d\right|_{\varphi(\bar{B})} .
$$

$$
\psi\left(\bar{f}_{x}\right)=\bar{F}_{x}
$$

then

$$
\bar{f}_{x}=\varphi \circ \psi\left(\bar{f}_{x}\right)=\frac{T_{Y} \bar{F}_{x}}{\bar{F}_{x}}
$$


The composition

$$
x \rightarrow f_{x} \rightarrow \bar{f}_{x} \rightarrow \bar{F}_{x} \rightarrow F_{x}
$$

gives a measurable choice of $F_{x}$, and $g_{x}$ is measurable as a quotient of measurable functions.

3.13. Remark. If $g_{x}(z) \in B\left(Y^{0}, *\right)\left(g_{x}\right.$ is constant), then the same proof works to give a measurable choice of $g_{x}, F_{x}$.

3.14. Notation. We write $f \sim g$ if $f / g=$ const.

3.15. Lemma. Let $X=Y \times_{\rho} H$ be an ergodic abelian extension of $Y$. Let $\sigma$ : $Y^{0} \times H \rightarrow S^{1}$ be such that for all $u \in H$ there exists a measurable function $f_{u}$ : $Y^{0} \times H \rightarrow S^{1}$ and a constant $\lambda_{u}$ such that

$$
\frac{\sigma(y, h+u)}{\sigma(y, h)}=\lambda_{u} \frac{f_{u}\left(T_{X}(y, h)\right)}{f_{u}(y, h)} .
$$

Then there exists a measurable family of measurable functions $\left\{f_{u}\right\}_{u \in H}$, a measurable family of constants $\left\{\lambda_{u}\right\}_{u \in H}$ satisfying the above equation, and a neighborhood $U$ of zero in $H$ such that

$$
\begin{aligned}
f_{u_{1}+u_{2}}(y, h) & \sim f_{u_{2}}\left(y, h+u_{1}\right) f_{u_{1}}(y, h), \\
\lambda_{u_{1}+u_{2}} & =\lambda_{u_{1}} \lambda_{u_{2}}
\end{aligned}
$$

whenever $u_{1}, u_{2}, u_{1}+u_{2} \in U$.

Proof. By Remark 3.13 we may assume that the families $\left\{f_{u}\right\}_{u \in H}$, and $\left\{\lambda_{u}\right\}_{u \in H}$ depend measurably on $u$. Using equation (13) we get

$$
\begin{aligned}
\frac{\sigma\left(y, h+u_{1}+u_{2}\right)}{\sigma(y, h)} & =\lambda_{u_{1}+u_{2}} \frac{T_{X} f_{u_{1}+u_{2}}(y, h)}{f_{u_{1}+u_{2}}(y, h)} \\
& =\lambda_{u_{1}} \lambda_{u_{2}} \frac{T_{X} f_{u_{1}}\left(y, h+u_{2}\right)}{f_{u_{1}}\left(y, h+u_{2}\right)} \frac{T_{X} f_{u_{2}}(y, h)}{f_{u_{2}}(y, h)} .
\end{aligned}
$$

This implies that

$$
\frac{f_{u_{1}+u_{2}}(y, h)}{f_{u_{1}}\left(y, h+u_{2}\right) f_{u_{2}}(y, h)}
$$

is an eigenfunction of $T_{X}$ and that

$$
\frac{\lambda_{u_{1}} \lambda_{u_{2}}}{\lambda_{u_{1}+u_{2}}}
$$

is an eigenvalue. Let $Z$ be the Kronecker factor of $X, \pi: X^{0} \rightarrow Z^{0}$ the projection map, let $N$ parametrize $\hat{Z}$, and let $\psi_{N\left(u_{1}, u_{2}\right)}(z)$ be a character of $Z$ s.t.:

$$
\frac{f_{u_{1}+u_{2}}(y, h)}{f_{u_{2}}\left(y, h+u_{1}\right) f_{u_{1}}(y, h)} \sim \psi_{N\left(u_{1}, u_{2}\right)} \circ \pi(y, h)
$$

and

$$
\frac{\lambda_{u_{1}} \lambda_{u_{2}}}{\lambda_{u_{1}+u_{2}}}=\psi_{N\left(u_{1}, u_{2}\right)}(\alpha) .
$$

Any two characters taking the same value on $\alpha$ are the same. Therefore $\psi_{N\left(u_{1}, u_{2}\right)}$ is symmetric, i.e.,

$$
\psi_{N\left(u_{1}, u_{2}\right)}=\psi_{N\left(u_{2}, u_{1}\right)}
$$


We now show that $\psi_{N\left(u_{1}, u_{2}\right)}$ satisfies a 2-cocycle equation:

$$
\begin{aligned}
\psi_{N\left(u_{1}+u_{2}, u_{3}\right)} \circ \pi(y, h) & \sim \frac{f_{u_{1}+u_{2}+u_{3}}(y, h)}{f_{u_{3}}\left(y, h+u_{1}+u_{2}\right) f_{u_{1}+u_{2}}(y, h)}, \\
\psi_{N\left(u_{1}, u_{2}+u_{3}\right)} \circ \pi(y, h) & \sim \frac{f_{u_{1}+u_{2}+u_{3}}(y, h)}{f_{u_{2}+u_{3}}\left(y, h+u_{1}\right) f_{u_{1}}(y, h)} .
\end{aligned}
$$

Thus

$$
\begin{aligned}
\left(\psi_{N\left(u_{1}+u_{2}, u_{3}\right)} \circ \pi(y, h)\right) f_{u_{3}}\left(y, h+u_{1}+u_{2}\right) f_{u_{1}+u_{2}}(y, h) & \\
& \sim\left(\psi_{N\left(u_{1}, u_{2}+u_{3}\right)} \circ \pi(y, h)\right) f_{u_{2}+u_{3}}\left(y, h+u_{1}\right) f_{u_{1}}(y, h) .
\end{aligned}
$$

Dividing both sides by

$$
f_{u_{1}}(y, h) f_{u_{3}}\left(y, h+u_{1}+u_{2}\right) f_{u_{2}}\left(y, h+u_{1}\right)
$$

we get

$$
\begin{aligned}
& \left(\psi_{N\left(u_{1}+u_{2}, u_{3}\right)} \circ \pi(y, h)\right) \frac{f_{u_{1}+u_{2}}(y, h)}{f_{u_{1}}(y, h) f_{u_{2}}\left(y, h+u_{1}\right)} \\
& \sim\left(\psi_{N\left(u_{1}, u_{2}+u_{3}\right)} \circ \pi(y, h)\right) \frac{f_{u_{2}+u_{3}}\left(y, h+u_{1}\right)}{f_{u_{2}}\left(y, h+u_{1}\right) f_{u_{3}}\left(y, h+u_{1}+u_{2}\right)} .
\end{aligned}
$$

Combining the above equation with equation (14),

$$
\psi_{N\left(u_{1}+u_{2}, u_{3}\right)} \psi_{N\left(u_{1}, u_{2}\right)}=\psi_{N\left(u_{1}, u_{2}+u_{3}\right)} \psi_{N\left(u_{2}, u_{3}\right)} .
$$

As $u \rightarrow f_{u}$ is a measurable function, $f_{u_{2}}(y), f_{u_{2}+u_{1}}(y)$ are close in measure for small $u_{1}$, most $u_{2}$, and the same goes for $f_{u_{2}}(y, h), f_{u_{2}}\left(y, h+u_{1}\right)$. Therefore the expression in equation (14) is close (in measure) to $f_{u_{1}}(y, h)$. But $N_{1} \neq N_{2}$ implies that

$$
\left\|\psi_{N_{1}}-\psi_{N_{2}}\right\|_{2}=\sqrt{2}
$$

thus by equation (14), $\psi_{N\left(u_{1}, u_{2}\right)}=\psi_{\tilde{N}\left(u_{1}\right)}$ for $u_{1} \in U^{\prime}$ a neighborhood of zero in $H$, $u_{2} \in A$ a set of positive measure. The set $A-A$ contains a neighborhood of zero $U^{\prime \prime}$. Let $U=U^{\prime} \cap U^{\prime \prime}$. Take any $u_{1}, u_{2}, u_{1}+u_{2} \in U$, and find an element $u_{3} \in A$ such that $u_{3}+u_{2} \in A$. Then by (16)

$$
\psi_{N\left(u_{1}, u_{2}\right)}=\psi_{\tilde{N}\left(u_{1}\right)} \psi_{\tilde{N}\left(u_{1}+u_{2}\right)}^{-1} \psi_{\tilde{N}\left(u_{2}\right)} .
$$

For $u \in U$, denote

$$
\tilde{f}_{u}(y, h)=\left(\psi_{\tilde{N}(u)} \circ \pi(y, h)\right) f_{u}(y, h),
$$

and

$$
\tilde{\lambda}_{u}=\lambda_{u} \psi_{\tilde{N}(u)}^{-1}(\alpha) .
$$

By equations (14), if $u_{1}, u_{2}, u_{1}+u_{2} \in U$, then

$$
\tilde{f}_{u_{1}+u_{2}}(y, h) \sim \tilde{f}_{u_{2}}\left(y, h+u_{1}\right) \tilde{f}_{u_{1}}(y, h) .
$$

By equation (15), if $u_{1}, u_{2}, u_{1}+u_{2} \in U$, then

$$
\tilde{\lambda}_{u_{1}+u_{2}}=\tilde{\lambda}_{u_{1}} \tilde{\lambda}_{u_{2}} .
$$

3.16. Lemma. Let $H$ be a torus (possibly infinite dimensional) and let $X=Y \times{ }_{\rho} H$ be an ergodic abelian extension of $Y$. Let $\sigma: Y^{0} \times H \rightarrow S^{1}$ be such that for all $u \in H$ there exists a measurable function $f_{u}: Y^{0} \times H \rightarrow S^{1}$ and a constant $\lambda_{u}$ such that

$$
\frac{\sigma(y, h+u)}{\sigma(y, h)}=\lambda_{u} \frac{f_{u}\left(T_{X}(y, h)\right)}{f_{u}(y, h)}
$$


and $\lambda_{u}$ and $f_{u}$ depend measurably on $u$. Then there is a closed subgroup $J<H$ such that $H / J=\mathbb{T}^{n}$ and such that if $\pi: H \rightarrow H / J$ is the natural projection, then there exists a function $\tilde{\sigma}: Y^{0} \times(H / J) \rightarrow S^{1}$ such that

$$
\sigma(y, h)=\tilde{\sigma}(y, \pi(h)) \frac{F\left(T_{X}(y, h)\right)}{F(y, h)} .
$$

Proof. By Lemma 3.15 the functions $f_{u}$ can be chosen such that $\lambda_{u}$ is multiplicative in a zero neighborhood $U$ in $H$. The neighborhood $U$ contains $J_{1}$, a closed connected subgroup of $H$, such that $H / J_{1}=\mathbb{T}^{l}$. Restricted to $J_{1}, \lambda_{u}$ is a character. Thinking of $H$ (measurably) as $H / J_{1} \times J_{1}$ with coordinates $\left(h_{0}, j\right)$ the above equation becomes

$$
\frac{\sigma\left(y, h_{0}, j+u\right)}{\sigma(y, h)}=\lambda_{u} \frac{f_{u}(T y, h+\rho(y))}{f_{u}(y, h)},
$$

where $u \in J_{1}$. This is the same as

$$
\frac{\lambda_{j+u}^{-1} \sigma\left(y, h_{0}, j+u\right)}{\lambda_{j}^{-1} \sigma(y, h)}=\frac{f_{u}(T y, h+\rho(y))}{f_{u}(y, h)} .
$$

Applying Corollary 3.9 and replacing $Y$ with $\left(Y \times H / J_{1}\right)$ and $H$ with $J_{1}$ we get

$$
\lambda_{j}^{-1} \sigma\left(y, h_{0}, j\right)=\tilde{\sigma}\left(y, h_{0}\right) \frac{T_{\rho} F(y, h)}{F(y, h)}
$$

or

$$
\sigma\left(y, h_{0}, j\right)=\lambda_{j} \tilde{\sigma}\left(y, h_{0}\right) \frac{T_{\rho} F(y, h)}{F(y, h)} .
$$

Now for $j$ in the kernel of $\lambda$ we have $\lambda_{j}=1$. The image of $\lambda$ is $S^{1}$; thus if $\operatorname{ker} \lambda$ is $J$, then $H / J=\mathbb{T}^{l+1}$.

3.17. Remark. The group $H$ is a compact connected abelian (metrizable) group and therefore has countably many closed subgroups $J$ such that $H / J$ is a finitedimensional torus.

3.18. Remark. If $H$ is any connected compact abelian group (not necessarily a torus), then $J_{1}$ in the foregoing proof is not necessarily connected. By the same proof we will get that $\sigma$ is cohomologous to a cocycle lifted from a product of a finite torus and a totally disconnected compact abelian group.

3.19. Lemma. Let $X=Y \times{ }_{\rho} H$ be an abelian extension of $Y$ with $\rho(y)$ cohomologous to a constant function (now $H$ is any compact abelian group, not necessarily connected). Let $\sigma$ be as in Lemma 3.16. Then there is a subgroup $J<H$, and a finite group $C_{k}$, such that $H / J=\mathbb{T}^{n} \times C_{k}$ and letting $\pi: H \rightarrow H / J$ denote the natural projection, then there exists a function $\tilde{\sigma}: Y^{0} \times H / J \rightarrow S^{1}$ such that

$$
\sigma(y, h)=\tilde{\sigma}(y, \pi(h)) \frac{T_{X} F(y, h)}{F(y, h)} .
$$

Proof. By Lemma 3.15 the functions $f_{u}$ can be chosen such that $\lambda_{u}$ is multiplicative in a zero neighborhood $U$ in $H$. The neighborhood $U$ contains $J_{1}$, a closed subgroup of $H$, such that $H / J_{1}=\mathbb{T}^{l} \times C_{j}$, where $C_{j}$ is a finite group; thus $\lambda_{u}$ is a character of $J_{1}$. Now proceed as in Lemma 3.16 (the image of $\lambda$ is either $S^{1}$ or a finite group). 


\section{LIE GROUPS AND NILSYSTEMS}

4.1. Let $N$ be a $k$-step, simply connected nilpotent Lie group, $\Gamma$ a discrete subgroup s.t. $N / \Gamma$ is compact. Let $\mathcal{B}$ be the (completed) Borel algebra, $m$ the Haar measure on $N / \Gamma$, and let $a \in N$. The system $X=(N / \Gamma, \mathcal{B}, m, T)$ where $T g \Gamma=a g \Gamma$ is called a nilflow. We will sometimes denote this system $(N / \Gamma, a)$. We will assume that the system $X$ is ergodic. Let $N_{1}=N$, and for $i>1: N_{i}=\left[N_{i-1}, N\right](N$ is a $k$-step nilpotent group if $N_{k+1}=\{1\}$ ), and for $i \geq 1$ let $\Gamma_{i}=\Gamma \cap N_{i}$. The groups $N_{j}$ for $j>1$ are connected (see L05]). The group $N_{k}$ is abelian and connected and therefore isomorphic to $\mathbb{R}^{m}$ for some positive integer $m$. Then $\Gamma_{i}$ is a discrete subgroup of $N_{i}$, and $N_{i} / \Gamma_{i}$ is compact. Let $m_{i}$ be the Haar measure on $N_{i} / \Gamma_{i}$, and let $l$ be a positive integer. Let

$$
M=\left\{\left(y_{1}, y_{1}^{2} y_{2}, \ldots, \prod_{j=1}^{l} y_{j}^{\left(\begin{array}{l}
l \\
j
\end{array}\right)}\right): y_{1} \in N_{1}, y_{2} \in N_{2}, \ldots, y_{l} \in N_{l}\right\} \subset N^{l}
$$

where

$$
\prod_{j=1}^{k} y_{j}^{\left(\begin{array}{l}
l \\
j
\end{array}\right)}=y_{1}^{l} y_{2}^{\left(\begin{array}{l}
l \\
2
\end{array}\right)} \ldots y_{k}^{\left(\begin{array}{l}
l \\
k
\end{array}\right)}
$$

$\left(N_{j}=\{1\}\right.$ for $\left.j>k\right)$. The elements of $M$ are called Hall-Petresco (HP) sequences and form a group (see [La54, L98]). The first $k+1$ elements in the sequence determine the rest. Computation shows that if $n \in N$ and $\left(n_{1}, \ldots, n_{l}\right) \in M$, then $\left(\left[n, n_{1}\right], \ldots,\left[n, n_{l}\right]\right) \in M$. Let $\Lambda=M \cap \Gamma^{l}$. The nilmanifold $Y=M / \Lambda$ is embedded in $(N / \Gamma)^{l}$, and let $\nu$ be the Haar measure on $Y$. Let $f_{1}, \ldots, f_{l}$ be bounded functions. Then for almost all $g \in N$ for all $\left(n_{1}, \ldots, n_{l}\right) \in M$,

$$
\lim _{N \rightarrow \infty} \frac{1}{N} \sum_{n=1}^{N} \prod_{j=1}^{l} f_{j}\left(a^{j n} n_{j} g \Gamma\right)=\int_{Y} \prod_{j=1}^{l} f_{j}\left(g z_{j}\right) d \nu\left(z_{1}, \ldots, z_{l}\right) .
$$

For more details, see [Z05]. A similar result holds for the action of $\left(a^{r_{1}}, \ldots, a^{r_{l}}\right)$ for any distinct $r_{i} \in \mathbb{Z}$.

4.2. Remark. If $N / \Gamma$ is connected, then $a^{k}$ is ergodic for any $k \neq 0$. Therefore equation (22) remains the same if we replace $a$ by $a^{k}$.

4.3. Let $(N / \Gamma, a)$ be a $k$-step nilflow. Define

$$
\tau_{l}(a):=a \times \ldots \times a^{l}, \quad \triangle_{l}(a):=a \times \ldots \times a .
$$

Let $\triangle_{l}(m)$ be the diagonal measure on $(N / \Gamma)^{l}$.

Define a measure on $(N / \Gamma)^{l}$ by

$$
\bar{\triangle}_{l}(m):=\lim _{N \rightarrow \infty} \frac{1}{N} \sum_{n=1}^{N} \tau_{l}(a)^{n} \triangle_{l}(m)
$$

By the above discussion the ergodic components of $\bar{\triangle}_{l}(m)$ are parametrized by $N / \Gamma$ and are (a.e. $g$ ) of the form $M(g \Gamma, \ldots, g \Gamma)$.

4.4. The system $(N / \Gamma, a)$ may be represented as an Abelian extension of a $(k-1)$ step nilflow $(N / \Gamma, a)=\left(N / N_{k} \Gamma \times{ }_{\rho} N_{k} / \Gamma_{k}\right)$. Inductively $(N / \Gamma, a)$ may be represented as a tower of Abelian extensions, starting out with a point. (The first block in the tower would be the Kronecker factor $N / N_{2} \Gamma$.) Consider the system $Y=\left(\left(N / N_{k} \Gamma\right)^{l}, \tau_{l}(a), \bar{\triangle}_{l}\right)$. Then $\left(Y \times_{\left(\rho^{(1)}, \ldots, \rho^{(l)}\right)}\left(N_{k} / \Gamma_{k}\right)^{l}, \tau_{l}(a)\right)$ is an abelian 
group extension of $Y$. The Mackey group, associated with the ergodic components of the group extension that are mapped onto the ergodic components of $\bar{\triangle}_{l}$, is

$$
M_{k, l}=\left\{\left(g_{1}, g_{1}^{2} g_{2}, \ldots, \prod_{j=1}^{k} g_{j}^{\left(\begin{array}{l}
l \\
j
\end{array}\right)}\right): g_{1}, \ldots, g_{k} \in N_{k}\right\} / \Gamma_{k}^{l} .
$$

In additive notation, denote $H=N_{k} / \Gamma_{k}$. Then

$$
M_{k, l}=\left\{\left(h_{1}, 2 h_{1}+h_{2}, \ldots, \sum_{j=1}^{k}\left(\begin{array}{l}
l \\
j
\end{array}\right) h_{j}\right): h_{1}, \ldots, h_{k} \in H\right\} .
$$

4.5. Lemma. Let $X=(N / \Gamma, a)$ be an ergodic $k$-step nilflow. Then for $r \geq 2$, $Y_{r}(X)=\left(N / N_{r} \Gamma, a\right)$.

Proof. We prove this by induction on the nilpotency level of $N$. Let $N$ be a 1-step nilpotent group (thus $(N / \Gamma, a)$ is a Kronecker system). Let $\psi$ be an eigenfunction of $(N / \Gamma, a)$. Then

$$
\frac{1}{N} \sum_{n=1}^{N} T^{n}(\psi)^{2} T^{2 n}(\psi)^{-1}=\psi
$$

Therefore

$$
L^{2}\left(Y_{1}\right)=\operatorname{span}\{\text { eigenfunctions }\}=L^{2}(N / \Gamma) .
$$

Now assume the statement for $(k-1)$-step nilflows. Let $X=(N / \Gamma, a)$ be a $k$-step nilflow. By Corollary 2.4 $Y_{r}\left(N / N_{r} \Gamma, a\right)$ is a factor of $Y_{r}(X)$. By the induction hypothesis $Y_{r}\left(N / N_{r} \Gamma, a\right)=\left(N / N_{r} \Gamma, a\right)$. But the integral in equation (22) for $l=r$ is a function on $N / \Gamma$ that is invariant under translation by elements of $N_{r}$. By Lemma 2.3. $L^{2}\left(Y_{r}(X)\right)$ is spanned by these integrals. Let $r=k+1$, and let $f(g \Gamma) \in L^{\infty}(N / \Gamma)$. We want to show that the function $f$ is in the span of the integrals one obtains in (22) when $n_{j}=1$ for $j=1, \ldots, k+1$. As the value of the limit in (22) is the same for any choice of $\left(n_{1}, \ldots, n_{k+1}\right) \in M$ we may consider the averages in (22) as averages over functions $f_{1} \otimes \ldots \otimes f_{k+1} \in L^{\infty}\left(\bar{\triangle}_{k+1}(m)\right)$. The element $g \Gamma$ is determined by the first $k+1$ elements $m_{1} g \Gamma, \ldots, m_{k+1} g \Gamma$ of an HP geometric progression (see [L98]). Therefore there exists a function $F \in$ $L^{\infty}\left(\bar{\triangle}_{k+1}(m)\right)$ such that

$$
F\left(m_{1} g \Gamma, \ldots, m_{k+1} g \Gamma\right)=f(g \Gamma) .
$$

Now as

$$
F\left(m_{1} g \Gamma, \ldots, m_{k+1} g \Gamma\right)=F\left(a m_{1} g \Gamma, \ldots, a^{k+1} m_{k+1} g \Gamma\right)
$$

we get

$$
\lim _{N \rightarrow \infty} \frac{1}{N} \sum_{n=1}^{N} \tau_{k+1}(a)^{n} F\left(m_{1} g \Gamma, \ldots, m_{k+1} g \Gamma\right)=f(g \Gamma) .
$$

Now approximate $F\left(\right.$ in $\left.L^{2}\left(\bar{\triangle}_{k+1}(m)\right)\right)$ by sums of functions of the type $f_{1} \otimes \ldots \otimes$ $f_{k+1}$.

4.6. Corollary (Theorem 1.8). If $X$ is an ergodic $k$-step pro-nilflow, then $Y_{k+1}(X)$ $=X$. 
4.7. Lemma. Let $(N / \Gamma, a)$ be a k-step nilflow. Let $f \in B\left(N / \Gamma, S^{1}\right)$. Let $\left\{\lambda_{c}\right\}$ be a family of constants, $\left\{f_{c}\right\}_{c \in N_{k}}$ be a family of functions in $B\left(N / \Gamma \rightarrow S^{1}\right)$ such that

$$
\frac{f(c y)}{f(y)}=\lambda_{c} \frac{f_{c}(a y)}{f_{c}(y)}
$$

for all $c \in N_{k}$. Then we can choose $f_{c}, \lambda_{c}$ such that

$$
f_{c_{1}}\left(c_{2} y\right) f_{c_{2}}(y) \sim f_{c_{1} c_{2}}(y) .
$$

Proof. By Lemma 3.15 this holds in a neighborhood of zero $U \subset N_{k}$. Notice that multiplying $f_{c}$ by a constant does not affect equation (24). If $c \in N_{k}, c=c_{1} \ldots c_{s}$, and $c_{1}, \ldots, c_{s} \in U$, define

$$
f_{c}(y)=f_{c_{1}}(y) f_{c_{2}}\left(c_{1} y\right) \ldots f_{c_{s}}\left(c_{1} \ldots c_{s-1} y\right) .
$$

We claim this is well defined (up to a constant multiple) on $N_{k}$ : given two sequences $c_{1}, \ldots c_{s}$ and $c_{1}^{\prime}, \ldots c_{t}^{\prime}$ with equal product, we can break up the "steps" $c_{r}$ into an equal number of small steps and we can interpolate a sequence of such paths where two consecutive paths differ only within a small cube which can be translated to be inside $U$. Since the resulting $\lambda$ 's and $f$ 's will be the same for consecutive paths, they will be the same for the initial and the final ones.

4.8. Lemma. Assume $N / \Gamma$ has no nontrivial finite factors. The group $N / N_{2} \cong \mathbb{R}^{n}$ or $N / N_{2} \cong \mathbb{Z} \times \mathbb{R}^{n}$ for some integer $n$. The action of a on it is given by rotation by some element $\alpha$ or $(1, \alpha)$ respectively. Under the conditions of Lemma 4.7 we can choose $f_{c}, \lambda_{c}$ so that

$$
\frac{f(c y)}{f(y)}=e^{2 \pi i\langle L \alpha, c\rangle} \frac{f_{c}(a y)}{f_{c}(y)},
$$

for an $n \times m$ integer matrix $L$.

Proof. We first treat the case where $N / N_{2} \cong \mathbb{Z} \times \mathbb{R}^{n}$. Now $\lambda_{c}$ is a continuous multiplicative function on $N_{k}=\mathbb{R}^{m}$. We now use additive notation for $N_{k}$. In this notation $\lambda_{c}$ is of the form $e^{2 \pi i\langle r, c\rangle}$. Let $e_{i}$ denote the standard basis for $\mathbb{R}^{m}$. Each $f_{e_{i}}$ is an eigenfunction (as the left side of equation (24) is 1). Thus there exists $\vec{n}_{i} \in \mathbb{Z}^{n}$ such that

$$
f_{e_{i}}(y)=C e^{2 \pi i\left\langle\vec{n}_{i}, \bar{y}\right\rangle},
$$

where $\bar{y} \in N / N_{2}$, with eigenvalue $e^{2 \pi i\left\langle\vec{n}_{i}, \alpha\right\rangle}$. Finally for each $i$ there is $k_{i} \in \mathbb{Z}$ such that

$$
\left\langle r, e_{i}\right\rangle=\left\langle\vec{n}_{i}, \alpha\right\rangle+k_{i} .
$$

Now take $L$ to be the matrix with the $i$ th row being $\left(k_{i}, \vec{n}_{i}\right)$ (the action of $a$ on $\mathbb{Z} \times \mathbb{R}^{n}$ given by $\left.(1, \alpha)\right)$. For the case $N / N_{2} \cong \mathbb{R}^{n}$ we get $\left\langle r, e_{i}\right\rangle=\left\langle\vec{n}_{i}, \alpha\right\rangle$ and take $L$ to be the matrix with the $i$ th row being $\left(\vec{n}_{i}\right)$.

4.9. Lemma. For any $c_{1}, c_{2} \in N_{k}, f_{c_{1}}$ and $f_{c_{2}}$ satisfying equation (24) we have

$$
\frac{f_{c_{1}}\left(c_{2} y\right)}{f_{c_{1}}(y)}=\frac{f_{c_{2}}\left(c_{1} y\right)}{f_{c_{2}}(y)} \text {. }
$$

Proof. The function

$$
\frac{f_{c_{1}}\left(c_{2} y\right)}{f_{c_{1}}(y)} / \frac{f_{c_{2}}\left(c_{1} y\right)}{f_{c_{2}}(y)}
$$


is invariant under rotation by $a$. Therefore

$$
\frac{f_{c_{1}}\left(c_{2} y\right)}{f_{c_{1}}(y)}=C\left(c_{1}, c_{2}\right) \frac{f_{c_{2}}\left(c_{1} y\right)}{f_{c_{2}}(y)} .
$$

Using Lemma 4.7 .

$$
\begin{aligned}
& C\left(c_{1}, c_{2} c\right) \frac{f_{c_{2}}\left(c_{1} c y\right) f_{c}\left(c_{1} y\right)}{f_{c_{2}}(c y) f_{c}(y)}=C\left(c_{1}, c_{2} c\right) \frac{f_{c_{2} c}\left(c_{1} y\right)}{f_{c_{2} c}(y)}=\frac{f_{c_{1}}\left(c_{2} c y\right)}{f_{c_{1}}(y)} \\
&=\frac{f_{c_{1}}\left(c_{2} c y\right)}{f_{c_{1}}(c y)} \frac{f_{c_{1}}(c y)}{f_{c_{1}}(y)}=C\left(c_{1}, c_{2}\right) C\left(c_{1}, c\right) \frac{f_{c_{2}}\left(c_{1} c y\right)}{f_{c_{2}}(y)} \frac{f_{c}\left(c_{1} y\right)}{f_{c}(y)} .
\end{aligned}
$$

Therefore $C\left(c_{1}, c_{2}\right)$ is multiplicative in $c_{1}, c_{2}$. If $c \in N_{k} \cap \Gamma$, then $f_{c}$ is an eigenfunction. Thus for $c \in N_{k} \cap \Gamma, C\left(c_{1}, c\right)=C\left(c, c_{2}\right)=1$. This implies that for any $c_{1} \in N_{k}, C\left(c_{1}, *\right)$ is a character of $N_{k} /\left(N_{k} \cap \Gamma\right)$, which is a compact connected abelian group. As there are countably many of those, $C\left(c_{1}, c_{2}\right) \equiv 1$.

4.10. Definition. Let $X=\left(X^{0}, \mathcal{B}, \mu, T\right)$ be an m.p.s. Let $\mathcal{A} \subset \mathcal{B}$ be a $T$-invariant sub- $\sigma$-algebra. If

$$
\mathcal{F}=\{f: f \text { measurable, }|f|=1, T f / f \text { is } \mathcal{A} \text { measurable }\},
$$

then we define $D(\mathcal{A})$ as the smallest $\sigma$-algebra with respect to which the functions of $\mathcal{F}$ are measurable, and define $D_{n}(\mathcal{A})=D\left(D_{n-1}(\mathcal{A})\right.$ ), where $D_{0}(\mathcal{A})=D(\mathcal{A})$. $T$ is said to have generalized discrete spectrum [mod $\mathcal{A}]$ of finite type if for some $n \in \mathbb{N}, D_{n}(\mathcal{N})\left[D_{n}(\mathcal{A})\right]$ is $\mathcal{B}$, where $\mathcal{N}$ is the trivial $\sigma$-algebra of null sets and their complements. Since $D_{n}(\mathcal{N}) \subset D_{n}(\mathcal{A})$, generalized discrete spectrum of finite type implies generalized discrete spectrum $\bmod \mathcal{A}$ of finite type. The qualification "generalized" is dropped when $n=1$.

4.11. Example. If $\left(Z \times H, T_{\rho}\right)$ is an Abelian extension of the Kronecker system $(Z, T)$, then $T_{\rho}$ has discrete spectrum of finite type mod the Kronecker algebra, and generalized discrete spectrum of finite type (mod the trivial algebra). Another example: if $(N / \Gamma, T)$ is a nilflow, then $T$ has generalized discrete spectrum of finite type.

4.12. Proposition (Parry [P73]). If $T$ is ergodic with discrete spectrum mod $\mathcal{A}$, then there exists a compact Abelian group $G$ of measure-preserving transformations such that $T(g x)=g T x$ for $g \in G$ and $\mathcal{A}=\{B \in \mathcal{B}: g B=B \forall g \in G\}$.

\section{The van der Corput lemma}

One of the main tools in studying characteristic factors is the van der Corput lemma. The formulation below is due to Bergelson [Be87:

5.1. Lemma (van der Corput). Let $\left\{u_{n}\right\}$ be a bounded sequence of vectors in a Hilbert space $\mathcal{H}$. Assume that for each $m$ the limit

$$
\gamma_{m}:=\lim _{N \rightarrow \infty} \frac{1}{N} \sum_{n=1}^{N}\left\langle u_{n}, u_{n+m}\right\rangle
$$


exists, and

$$
\lim _{M \rightarrow \infty} \frac{1}{M} \sum_{m=1}^{M} \gamma_{m}=0
$$

Then

$$
\frac{1}{N} \sum_{n=1}^{N} u_{n} \stackrel{\mathcal{H}}{\longrightarrow} 0
$$

Proof. Let $M$ be large enough so that the expression in (25) is small. Let $N$ be large enough with respect to $M$ so that the two expressions

$$
\frac{1}{N M} \sum_{n=1}^{N} \sum_{m=1}^{M} u_{n+m}, \quad \frac{1}{N} \sum_{n=1}^{N} u_{n}
$$

are close. We have:

$$
\begin{aligned}
& \left\|\frac{1}{N M} \sum_{n=1}^{N} \sum_{m=1}^{M} u_{n+m}\right\|^{2} \leq \frac{1}{N} \sum_{n=1}^{N}\left\|\frac{1}{M} \sum_{m=1}^{M} u_{n+m}\right\|^{2} \\
& =\frac{1}{N M^{2}} \sum_{n=1}^{N} \sum_{m_{1}, m_{2}=1}^{M}\left\langle u_{n+m_{1}}, u_{n+m_{2}}\right\rangle \stackrel{N \rightarrow \infty}{\longrightarrow} \frac{1}{M^{2}} \sum_{m_{1}, m_{2}=1}^{M} \gamma_{m_{2}-m_{1}},
\end{aligned}
$$

which is small.

\section{Proof of Theorem 1.7}

Let $X$ be an ergodic m.p.s., and let $Y_{j}(X)$ be the $j$-u.c.f. of $X$, and let $\pi_{j}: X \rightarrow$ $Y_{j}(X)$ be the factor map. When the context is clear we will write $T$ for $T_{X}$, and $Y_{j}$ for $Y_{j}(X)$. Let $\vec{a}=\left(a_{1}, \ldots, a_{l}\right) \in \mathbb{Z}^{l}$. We will always assume that the $a_{i}$ are distinct. Denote

$$
\begin{aligned}
\tau_{\vec{a}}(T) & :=T^{a_{1}} \times \ldots \times T^{a_{l}}, \\
\triangle_{l}(T) & :=T \times \ldots \times T .
\end{aligned}
$$

When the context is clear we will use $\tau_{\vec{a}}$ for $\tau_{\vec{a}}(T)$, and $T$ or $T_{l}$ for $\triangle_{l}(T)$. Let $\triangle_{l}\left(\mu_{X}\right)$ be the diagonal measure on $\left(X^{0}\right)^{l}$. We will prove Theorem 1.7 inductively, along with a sequence of statements (Theorem 1.7] is item (7)).

6.1. Theorem. (1) Let $\vec{a}=\left(a_{1}, \ldots, a_{j+1}\right) \in \mathbb{Z}^{j+1}$. The limit

$$
\bar{\triangle}_{\vec{a}}\left(\mu_{X}\right):=\lim _{N \rightarrow \infty} \frac{1}{N} \sum_{n=1}^{N} \tau_{\vec{a}}^{n} \triangle_{j+1}\left(\mu_{X}\right)
$$

exists. (The convergence here is weak convergence relative to the algebra of 
functions on the product space spanned by products of bounded measurable functions.) Furthermore $\bar{\triangle}_{\vec{a}}\left(\mu_{X}\right)$ is the conditional product measure relative to $\bar{\triangle}_{\vec{a}}\left(\mu_{Y_{j}}\right)$.

(2) $Y_{j+1}(X)$ is an isometric extension of $Y_{j}(X)$.

(3) Let $X$ be an ergodic m.p.s. Let $l \in \mathbb{N}$. Let $\mu$ be a measure on $\left(X^{0}\right)^{l}$, let $\vec{a}=\left(a_{1}, \ldots, a_{l}\right) \in \mathbb{Z}^{l}$, and for $i=1, \ldots, l$, let $f_{i} \in B\left(X^{0}, S^{1}\right)$. Recall that $f^{(m)}(x)=f\left(T^{m-1} x\right) \ldots f(T x) f(x)$. We say that $\left(f_{1}, f_{2}, \ldots, f_{l}\right)$ is of type $\vec{a}$ w.r.t. $\mu$ if there exists a $\mu$-measurable function $F$ taking values in $S^{1}$, such that $\mu$ almost everywhere

$$
\prod_{i=1}^{l} f_{i}^{\left(a_{i}\right)}\left(x_{i}\right)=\frac{\tau_{\vec{a}} F\left(x_{1}, \ldots, x_{l}\right)}{F\left(x_{1}, \ldots, x_{l}\right)} .
$$

Let $H$ be a compact abelian group, $Y$ a $(j-1)$-step pro-nilflow. We say that $\rho: Y \rightarrow H$ is of type $j$ if for any character $\chi \in \hat{H}$, there exists an integer $l$, a character $\tilde{\chi}=\left(\chi_{1}, \ldots, \chi_{l}\right) \in \hat{H}^{l}$, and $\vec{a} \in \mathbb{Z}^{l}$, such that $\chi=\chi_{k}$ for some $l \geq k \geq 1$ and $\left(\chi_{1} \circ \rho, \ldots, \chi_{l} \circ \rho\right)$ is of type $\vec{a}$ w.r.t. $\bar{\triangle}_{\vec{a}}\left(\mu_{Y}\right)$. Let $Y$ be a $(j-1)$-step pro-nilflow, and let $\left(f_{1}, f_{2}, \ldots, f_{l}\right)$ be of type $\vec{a}$ w.r.t. $\bar{\triangle}_{\vec{a}}\left(\mu_{Y}\right)$. Then

(a) $f_{k}$ is cohomologous to a function lifted from a $(j-1)$-step nilflow.

(b) For $k=1, \ldots, l, f_{k}$ belongs to a countable set modulo coboundaries.

(c) For $k=1, \ldots, l, f_{k}: Y \rightarrow S^{1}$ is of type $j$.

(d) If $\rho: Y \rightarrow H$ is of type $j$, then for any character $\chi$ of $H, \chi \circ \rho$ is of type $j$.

(e) If $f, g: Y \rightarrow S^{1}$ are of type $j$, then $f g$ is of type $j$.

(4) If $X=Y_{j}(X) \times_{\sigma} H$ is an abelian extension by a cocycle of type $j$, then $X$ can be given the structure of a $j$-step pro-nilflow. If $Y_{j}(X)$ is a nilflow and $H$ is a finite-dimensional torus, then $X$ is a nilflow.

(5) A factor of a $j$-step pro-nilflow is a $j$-step pro-nilflow.

(6) If $X$ is a $j$-step pro-nilflow, then $X=Y_{j}(X) \times_{\sigma} H$ is an abelian extension of $Y_{j}(X)$ by a cocycle of type $j$. If $j \geq 1$, then $H$ is connected.

(7) $Y_{j+1}(X)$ can be given the structure of a $j$-step pro-nilflow.

(8) Let $a_{1}, \ldots, a_{j+1} \in \mathbb{Z}$, and $f_{1}, \ldots, f_{j+1} \in L^{\infty}\left(\mu_{X}\right)$. Then the averages

$$
\frac{1}{N} \sum_{n=1}^{N} \prod_{k=1}^{j+1} f_{k}\left(T^{a_{k} n} x\right)
$$

converge in $L^{2}\left(\mu_{X}\right)$.

Proof. For $j=0, Y_{j+1}(X)$ is the pro-cyclic factor. For $j=1, Y_{j+1}(X)$ is the Kronecker factor which is an abelian extension (by a connected group) of the procyclic factor by a constant cocycle, and all statements are easily verified. Assume all statements hold when replacing $j$ with $j-1$. 
6.2. Proof of Theorem 6.1(1):

$$
\begin{aligned}
\lim _{N \rightarrow \infty} & \frac{1}{N} \sum_{n=1}^{N} \int \tau_{\vec{a}}^{n} f_{1} \otimes \ldots \otimes f_{j+1} d \triangle_{j+1}\left(\mu_{X}\right) \\
& =\lim _{N \rightarrow \infty} \frac{1}{N} \sum_{n=1}^{N} \int f_{1}\left(T^{n a_{1}} x\right) f_{2}\left(T^{n a_{2}} x\right) \ldots f_{j+1}\left(T^{n a_{j+1}} x\right) d \mu_{X} \\
& =\lim _{N \rightarrow \infty} \frac{1}{N} \sum_{n=1}^{N} \int f_{1}(x) f_{2}\left(T^{n\left(a_{2}-a_{1}\right)} x\right) \ldots f_{j+1}\left(T^{n\left(a_{j+1}-a_{1}\right)} x\right) d \mu_{X} \\
& =\lim _{N \rightarrow \infty} \frac{1}{N} \sum_{n=1}^{N} \int E\left(f_{1} \mid Y_{j}\right)\left(\pi_{j} x\right) \prod_{i=1}^{j}\left(T^{n\left(a_{i+1}-a_{1}\right)} E\left(f_{i+1} \mid Y_{j}\right)\left(\pi_{j} x\right)\right) d \mu_{Y_{j}} \\
& =\int E\left(f_{1} \mid Y_{j}\right) \otimes \ldots \otimes E\left(f_{j+1} \mid Y_{j}\right) d \bar{\triangle}_{\vec{a}}\left(\mu_{Y_{j}}\right) .
\end{aligned}
$$

By the above calculation,

$$
\int f_{1} \otimes \ldots \otimes f_{j+1} d \bar{\triangle}_{\vec{a}}\left(\mu_{X}\right)=\int E\left(f_{1} \mid Y_{j}\right) \otimes \ldots \otimes E\left(f_{j+1} \mid Y_{j}\right) d \bar{\triangle}_{\vec{a}}\left(\mu_{Y_{j}}\right) ;
$$

thus $\bar{\triangle}_{\vec{a}}\left(\mu_{X}\right)$ is the conditional product measure relative to $\bar{\triangle}_{\vec{a}}\left(\mu_{Y_{j}}\right)$.

6.3. Proof of Theorem 6.1(2). We must show that if $E\left(f_{k} \mid \mathcal{B}_{\hat{Y}_{j}(X)}\right)=0$ for some $k$, then the averages $\frac{1}{N} \sum_{n=1}^{N} \prod_{k=1}^{j+1} f_{k}\left(T^{a_{k} n} x\right)$ converge to zero in $L^{2}\left(\mu_{X}\right)$. We apply the van der Corput Lemma 5.1 with

$$
u_{n}=\prod_{k=1}^{j+1} T^{n a_{k}} f_{k}(x) .
$$

We calculate $\gamma_{m}$ :

$$
\begin{aligned}
\gamma_{m} & =\lim \frac{1}{N} \sum_{n=1}^{N}\left\langle u_{n}, u_{n+m}\right\rangle \\
& =\lim \frac{1}{N} \sum_{n=1}^{N} \int \prod_{k=1}^{j+1} T^{n a_{k}} f_{k}(x) T^{n a_{k}+m a_{k}} f_{k}(x) d \mu_{X} \\
& =\lim \frac{1}{N} \sum_{n=1}^{N} \int \prod_{k=1}^{j+1} T^{n a_{k}}\left(f_{k} T^{m a_{k}} f_{k}(x)\right) d \mu_{X} \\
& =\int\left(f_{1} \otimes \ldots \otimes f_{j+1}\right) \tau_{\vec{a}}^{m}\left(f_{1} \otimes \ldots \otimes f_{j+1}\right) d \bar{\triangle}_{\vec{a}}\left(\mu_{X}\right) .
\end{aligned}
$$

By the ergodic theorem, there exists a $\tau_{\vec{a}}$-invariant function $D_{\vec{a}} \in L^{2}\left(\bar{\triangle}_{\vec{a}}\left(\mu_{X}\right)\right)$ such that

$$
\lim \frac{1}{M} \sum_{m=1}^{M} \gamma_{m}=\int f_{1} \otimes \ldots \otimes f_{j+1} D_{\vec{a}}\left(x_{1}, \ldots, x_{j+1}\right) d \bar{\triangle}_{\vec{a}}\left(\mu_{X}\right) .
$$

By 6.2, $\bar{\triangle}_{\vec{a}}\left(\mu_{X}\right)$ is the conditional product measure relative to $\bar{\triangle}_{\vec{a}}\left(\mu_{Y_{j}}\right)$. By Theorem 2.11, $D_{\vec{a}}$ is measurable w.r.t. $\left(\hat{Y}_{j}(X)\right)^{j+1}$. If $E\left(f_{k} \mid \mathcal{B}_{\hat{Y}_{j}(X)}\right)=0$, then the average (27) is zero, and by the van der Corput Lemma 5.1, so is the original average. 
6.4. Proof of Theorem 6.1(3). This part is the bulk of the theorem, and the proof of its items will be intertwined with the proof of the rest of the items in Theorem 6.1 Let $Y$ be a $(j-1)$-step pro-nilflow, with $j \geq 2$. By Corollary 4.6. $Y=Y_{j}(Y)$. By the induction hypothesis in Theorem 6.1 (6), we can identify $Y$ with a presentation as a tower of abelian extensions $Y=H_{1} \times_{\sigma_{1}} H_{2} \times \ldots \times_{\sigma_{j-1}} H_{j}$ where $\sigma_{i}$ is of type $i, H_{i}$ is connected for $i>1$, and $Y_{i}(Y)=H_{1} \times_{\sigma_{1}} H_{2} \times \ldots \times_{\sigma_{i-1}} H_{i}$. Specifically $Y=Y_{j-1}(Y) \times_{\sigma_{j-1}} H_{j}$, where $H_{j}$ is a connected compact abelian group, and $\sigma_{j-1}$ is of type $j-1$. Denote $Y_{j-1}=Y_{j-1}(Y)$. Let $\pi_{j-1}: Y \rightarrow Y_{j-1}$ be the projection. We identify $y \in Y$ with $\left(\pi_{j-1} y, h\right) \in \pi_{j-1} Y \times H_{j}$. Let $l$ be a positive integer. To simplify the notation we now restrict ourselves to the special case where $\vec{a}=(1,2, \ldots, l)$. The analysis is similar for any $\vec{a} \subset \mathbb{Z}^{l}$.

We write $\bar{\triangle}_{l}\left(\mu_{Y}\right)$ for the measure $\bar{\triangle}_{(1, \ldots, l)}\left(\mu_{Y}\right)$, and we say that $\left(f_{1}, \ldots, f_{l}\right)$ is of type $l$ w.r.t. $\bar{\triangle}_{l}\left(\mu_{Y}\right)$ if $\left(f_{1}, \ldots, f_{l}\right)$ is of type $(1, \ldots, l)$ w.r.t. $\bar{\triangle}_{l}\left(\mu_{Y}\right)$. In this case there exists a function $F \in L^{\infty}\left(\bar{\triangle}_{l}\left(\mu_{Y}\right)\right)$ such that

$$
\prod_{k=1}^{l} f_{k}^{(k)}\left(y_{k}\right)=\frac{\tau F\left(y_{1}, \ldots, y_{l}\right)}{F\left(y_{1}, \ldots, y_{l}\right)}
$$

6.5. Remark. As $Y$ is a $(j-1)$-step pro-nilflow, on the support of $\bar{\triangle}_{l}\left(\mu_{Y}\right)$, the coordinates $y_{j+1}, \ldots, y_{l}$ are determined by the first $j$ coordinates $y_{1}, \ldots, y_{j}$, and this correspondence is invariant under $\tau$ (if $j=2$, then $Y$ is an abelian group, and $y_{1}, y_{2}, y_{3}$ form an arithmetic sequence; in general see the discussion in 4.1). Therefore the function $F\left(y_{1}, \ldots, y_{l}\right)$ can be replaced by a function of $j$ coordinates, and equation (28) can be written in the form

$$
\prod_{k=1}^{l} f_{k}^{(k)}\left(y_{k}\right)=\frac{\tau F\left(y_{1}, \ldots, y_{j}\right)}{F\left(y_{1}, \ldots, y_{j}\right)}
$$

We will repeatedly refer to this equation.

6.6. Remark. The measure $\bar{\triangle}_{j}\left(\mu_{Y}\right)=\bar{\triangle}_{j}\left(\mu_{Y_{j-1}}\right) \times\left(m_{H: j}\right)^{j}$ (replace $X$ by $Y$ and $j$ by $j-1$ in equation (26) ).

6.7. Lemma. Let $\left(f_{1}, \ldots, f_{j+1}\right)$ be of type $j+1$ w.r.t. $\bar{\triangle}_{j+1}\left(\mu_{Y}\right)$. Then for each $k=1, \ldots, j+1$ there exists a family of functions $\left\{g_{k, u}\right\}_{u \in H_{j}} \subset B\left(\pi_{j-1} Y^{0}, S^{1}\right)$, and a family of functions $\left\{f_{k, u}\right\}_{u \in H_{j}} \subset B\left(Y^{0}, S^{1}\right)$ such that

$$
\frac{f_{k}\left(\pi_{j-1} y, h+u\right)}{f_{k}\left(\pi_{j-1} y, h\right)}=g_{k, u}\left(\pi_{j-1} y\right) \frac{T f_{k, u}(y)}{f_{k, u}(y)} .
$$

Proof. We use the fact that $\bar{\triangle}_{j+1}(Y)$ is invariant under translations by elements of the Mackey group $M_{j-1, j+1}$, and by elements of $\triangle_{j+1}\left(H_{j}\right)=\{(h, \ldots, h)\}_{h \in H_{j}} \subset$ $H_{j}^{j+1}$. This group is described in 4.4. Let

$$
M_{j-1, j+1}(0)=\left(M_{j-1, j+1}+\triangle_{j+1}\left(H_{j}\right)\right) \cap\left(H_{j}^{j} \times\{0\}\right) .
$$

The projection of $M_{j-1, j+1}(0)$ on any $j$ coordinates is full (i.e., $H_{j}^{j}$ ). Let $\vec{u}=$ $\left(u_{1}, \ldots, u_{j+1}\right) \in M_{j-1, j+1}(0)\left(u_{j+1}=0\right)$. Then

$$
\prod_{k=1}^{j+1} f_{k}^{(k)}\left(\pi_{j-1} y_{k}, h_{k}+u_{k}\right)=\frac{\tau F\left(\pi_{j-1} y_{1}, h_{1}+u_{1}, \ldots, \pi_{j-1} y_{j}, h_{j}+u_{j}\right)}{F\left(\pi_{j-1} y_{1}, h_{1}+u_{1}, \ldots, \pi_{j-1} y_{j}, h_{j}+u_{j}\right)} .
$$


Dividing equation (31) by equation (29) we get

$$
\prod_{k=1}^{j} \frac{f_{k}^{(k)}\left(\pi_{j-1} y_{k}, h_{k}+u_{k}\right)}{f_{k}^{(k)}\left(\pi_{j-1} y_{k}, h_{k}\right)}=\frac{\tau F_{\vec{u}}\left(y_{1}, \ldots, y_{j}\right)}{F_{\vec{u}}\left(y_{1}, \ldots, y_{j}\right)}
$$

where

$$
F_{\vec{u}}\left(y_{1}, \ldots, y_{j}\right)=\frac{F\left(\pi_{j-1} y_{1}, h_{1}+u_{1}, \ldots, \pi_{j-1} y_{j}, h_{j}+u_{j}\right)}{F\left(y_{1}, \ldots, y_{j+1}\right)} .
$$

Using Remark 6.6 we can apply Theorem 3.8 to $\bar{\triangle}_{j}\left(\mu_{Y}\right)$. For any $1 \leq k \leq j$ and any $u_{k} \in H_{j}$ there exist functions $g_{k, u_{k}} \in B\left(Y^{0}, S^{1}\right)$ and $f_{k, u_{k}} \in B\left(Y^{0}, S^{1}\right)$ such that

$$
\frac{f_{k}^{(k)}\left(\pi_{j-1} y, h+u_{k}\right)}{f_{k}^{(k)}\left(\pi_{j-1} y, h\right)}=g_{k, u_{k}}\left(\pi_{j-1} y\right) \frac{T^{k} f_{k, u_{k}}(y)}{f_{k, u_{k}}(y)} .
$$

We still need to show that the same holds for $f_{k}$ (rather than $f_{k}^{(k)}$ ): we use the fact that $T_{f_{k}}$ (see 2.7) and $T_{f_{k}^{(k)}}^{k}=\left(T_{f_{k}}\right)^{k}$ commute; therefore

$$
f_{k}^{(k)}(T y) f_{k}(y)=f_{k}\left(T^{k} y\right) f_{k}^{(k)}(y) .
$$

Using equations (33), (34), and a calculation we find that the function

$$
\frac{f_{k}\left(\pi_{j-1} y, h+u+v\right) / f_{k}\left(\pi_{j-1} y, h+u\right)}{f_{k}\left(\pi_{j-1} y, h+v\right) / f_{k}\left(\pi_{j-1} y, h\right)} / \frac{T\left(f_{k, v}\left(\pi_{j-1} y, h+u\right) / f_{k, v}\left(\pi_{j-1} y, h\right)\right)}{f_{k, v}\left(\pi_{j-1} y, h+u\right) / f_{k, v}\left(\pi_{j-1} y, h\right)}
$$

is $T^{k}$-invariant and therefore constant on the (finitely many) ergodic components of $T^{k}$. Denote this constant by $\delta_{k, u, v}\left(\pi_{1} y\right)$ (the ergodic components of $T^{k}$ are determined by $\left.Y_{1}(X)\right)$. By Lemma 3.15, $\delta_{k, u, v}\left(\pi_{1} y\right)$ is multiplicative in $u$ (also in $v$ ) in a neighborhood of zero in $H_{j}$. By equation (33) (after iteration), $\left(\delta_{k, u, v}\right)^{k}\left(\pi_{1} y\right)$ is an eigenvalue. Therefore $\delta_{k, u, v}\left(\pi_{1} y\right)=1$ for $u$ in a neighborhood of zero in $H_{j}$. Iterating we find this is true for all $u \in H_{j}$ ( $H_{j}$ is connected). By Corollary 3.9 there exist functions $\tilde{g}_{k, v}, \tilde{f}_{k, v}$ such that

$$
\frac{f_{k}\left(\pi_{j-1} y, h+v\right)}{f_{k}\left(\pi_{j-1} y, h\right)}=\tilde{g}_{k, v}\left(\pi_{j-1} y\right) \frac{T \tilde{f}_{k, v}\left(\pi_{j-1} y, h\right)}{\tilde{f}_{k, v}\left(\pi_{j-1} y, h\right)} .
$$

6.8. Lemma. If $\left(g_{1} \circ \pi_{j-1}, \ldots, g_{l} \circ \pi_{j-1}\right)$ is of type $l$ w.r.t. $\bar{\triangle}_{l}\left(\mu_{Y}\right)$, then $g_{k}$ : $\pi_{j-1} Y \rightarrow S^{1}$ is of type $j-1$, for $k=1, \ldots, l$.

Proof. By definition there exists a $\bar{\triangle}_{l}\left(\mu_{Y}\right)$ measurable function $L$ such that

$$
\prod_{k=1}^{l}\left(g_{k}\right)^{(k)}\left(\pi_{j-1} y_{k}\right)=\frac{\tau L\left(y_{1}, \ldots, y_{l}\right)}{L\left(y_{1}, \ldots, y_{l}\right)} .
$$

Taking the Fourier expansion of $L$ with respect to the abelian group $H_{j}^{l}$,

$$
L\left(y_{1}, \ldots, y_{l}\right)=\sum_{\chi \in \hat{H}_{j}^{j}} G_{\chi}\left(\pi_{j-1} y_{1}, \ldots, \pi_{j-1} y_{l}\right) \chi_{1}\left(h_{1}\right) \ldots \chi_{j}\left(h_{l}\right) .
$$

We find that for any $\chi \in \hat{H}_{j}^{l}$,

$$
G_{\chi}\left(y_{1}, \ldots, y_{l}\right) \prod_{k=1}^{l} g_{k}^{(k)}\left(\pi_{j-1} y_{k}\right) \bar{\chi}_{k}\left(\sigma_{j-1}^{(k)}\left(\pi_{j-1} y_{k}\right)\right)=\tau G_{\chi}\left(\pi_{j-1} y_{1}, \ldots, \pi_{j-1} y_{l}\right) .
$$


The function $\left|G_{\chi}\right|$ is invariant under $\tau$ and therefore constant on the ergodic components of $\bar{\triangle}_{l}\left(\mu_{Y_{j-1}}\right)$. As $\hat{H}_{j}^{l}$ is countable, there is a character $\chi \in \hat{H}_{j}^{l}$, and a set of $\bar{\triangle}_{l}\left(\mu_{Y_{j-1}}\right)$ positive measure $A$, that is $\tau$-invariant, and for which

$$
\prod_{k=1}^{l} g_{k}^{(k)}\left(\pi_{j-1} y_{k}\right) \bar{\chi}_{k} \sigma_{j-1}^{(k)}\left(\pi_{j-1} y_{k}\right)=\frac{\tau G_{\chi}\left(\pi_{j-1} y_{1}, \ldots, \pi_{j-1} y_{l}\right)}{G_{\chi}\left(\pi_{j-1} y_{1}, \ldots, \pi_{j-1} y_{l}\right)} .
$$

Denote by $W$ the system $\left(Y_{j-1}^{l}, \bar{\triangle}_{l}\left(\mu_{Y_{j-1}}\right), \tau\right)$. Let $\rho_{k}=g_{k} \bar{\chi}_{k}\left(\sigma_{j-1}\right): Y_{j-1} \rightarrow S^{1}$, and let $\tilde{\rho}=\left(\rho_{1}, \rho_{2}^{(2)}, \ldots, \rho_{l}^{(l)}\right)$. Denote $\bar{y}:=\pi_{j-1} y$. Consider the group extension $W \times_{\tilde{\rho}}\left(S^{1}\right)^{l}$. Let $W_{\bar{y}}$ be an ergodic component of $W$ (the ergodic components of $W$ are parametrized by $\left.\bar{y} \in Y_{j-1}\right)$, and let $P_{\bar{y}} \subset\left(S^{1}\right)^{l}$ be the Mackey group associated with the ergodic components of the group extension $W_{\bar{y}} \times_{\tilde{\rho}}\left(S^{1}\right)^{l}$. As the transformations

$$
T_{\rho_{1}} \times\left(T_{\rho_{2}}\right)^{2} \times \ldots \times\left(T_{\rho_{l}}\right)^{l}, \quad T_{\rho_{1}} \times T_{\rho_{2}} \times \ldots \times T_{\rho_{l}}
$$

commute, $P_{T \bar{y}}=P_{\bar{y}}$ (see Lemma 2.18), and by ergodicity, $P_{\bar{y}}=P$ is constant a.e. as a function of $\bar{y}$. As equation (35) holds on a $\tau$-invariant set of $\bar{\triangle}_{l}\left(\mu_{Y_{j-1}}\right)$ positive measure, we get

$$
(\varphi, \ldots, \varphi) \in P^{\perp}
$$

where $\varphi(\zeta)=\zeta$. Therefore for a.e. $\bar{y}$, there exists a function $G_{\bar{y}}$, so that equation (35) holds on $W_{\bar{y}}$ when replacing $G_{\chi}$ with $G_{\bar{y}}$. Notice that $G_{\bar{y}}$ is determined up to a constant multiple on $W_{\bar{y}}$ and can therefore be chosen so that it depends measurably on $\bar{y}$ (see 3.13). Thus there exists a measurable function $G$ such that equation (35) holds $\bar{\triangle}_{l}\left(\mu_{Y_{j-1}}\right)$ a.e. when replacing $G_{\chi}$ by $G$. This implies that $\left(g_{1} \cdot \bar{\chi}_{1}\left(\sigma_{j-1}\right), \ldots, g_{l} \cdot \bar{\chi}_{l}\left(\sigma_{j-1}\right)\right)$ is of type $l$ w.r.t. $\bar{\triangle}_{l}\left(\mu_{Y_{j-1}}\right)$. By the induction hypothesis in 6.1(3c), (3d) the functions $g_{k} \bar{\chi}_{k}\left(\sigma_{j-1}\right), \chi_{k}\left(\sigma_{j-1}\right)$ are of type $j-1$. By the induction hypothesis in 6.1(3e), $g_{k}$ is of type $j-1$.

6.9. Corollary. Let $\left(f_{1}, \ldots, f_{j+1}\right)$ be of type $j+1$ w.r.t. $\bar{\triangle}_{j+1}\left(\mu_{Y}\right)$. Let $\vec{u} \in$ $M_{j-1, j+1}(0)$, and let $g_{k, u_{k}}$ satisfy equation (30). Then for $k=1, \ldots, j+1, g_{k, u_{k}}$ is of type $j-1$.

Proof. Substitute equation (33) in equation (32) and use Lemma 6.8.

6.10. Corollary. If $\left(f_{1}, \ldots, f_{j+1}\right)$ is of type $j+1$ w.r.t. $\bar{\triangle}_{j+1}\left(\mu_{Y}\right)$, then for $k=$ $1, \ldots, j+1$ there exists a family of constants $\left\{\lambda_{k, u}\right\}_{u \in H_{j}}$, and a family of measurable functions $\left\{f_{k, u}\right\}_{u \in H_{j}}$ such that

$$
\frac{f_{k}\left(\pi_{j-1} y, h+u\right)}{f_{k}\left(\pi_{j-1} y, h\right)}=\lambda_{k, u} \frac{T f_{k, u}(y)}{f_{k, u}(y)} .
$$

Proof. By theorem 6.1(3b), using the induction hypothesis, there are countably many $g_{k, u}$ up to $\pi_{j-1} Y$-quasi-coboundaries. There exists a set $U$ of positive measure in $H_{j}$ such that

$$
u, v \in U \Rightarrow \frac{g_{k, u}}{g_{k, v}} \text { is a quasi-coboundary. }
$$

If $u, u+v \in U$ and if $f_{k, u, v}=f_{k, u+v} / f_{k, u}$, then

$$
\frac{f_{k}\left(\pi_{j-1} y, h+u+v\right)}{f_{k}\left(\pi_{j-1} y, h+u\right)}=\frac{f_{k}\left(\pi_{j-1} y, h+u+v\right) / f_{k}\left(\pi_{j-1} y, h\right)}{f_{k}\left(\pi_{j-1} y, h+u\right) / f_{k}\left(\pi_{j-1} y, h\right)}=C_{u, v, k} \frac{T f_{k, u, v}(y)}{f_{k, u, v}(y)} .
$$


Thus the claim is true for $v$ in a neighborhood of zero in $H_{j}$ (as the map sending $\left(\pi_{j-1} y, h\right)$ to $\left(\pi_{j-1} y, h+u\right)$ is onto and commutes with the $T$ action $)$. As $H_{j}$ is connected, equation (36) holds for all $v \in H_{j}$.

6.11. Lemma. The families in the previous lemma can be chosen so that the function $\lambda_{k, u}: H_{j} \rightarrow S^{1}$ is multiplicative in a neighborhood of zero in $H_{j}$.

Proof. By Lemma 3.15

6.12. Corollary. The functions $f_{k, u}$ from Corollary 6.10 can be chosen so that for some neighborhood of zero $U \subset H$, for any $\vec{u}=\left(u_{1}, \ldots, u_{j+1}\right) \in M_{j-1, j+1}+$ $\triangle_{j+1}\left(H_{j}\right) \cap U^{j+1}$,

$$
\prod_{k=1}^{j+1} \lambda_{k, u_{k}}^{k}=1 .
$$

Proof. Choose the families $\left\{f_{k, u}\right\},\left\{\lambda_{k, u}\right\}$ so that $\lambda_{k, u}$ is multiplicative in a a neighborhood of zero in $H_{j}$. Substituting equation (36) in equation (32), we find that $\prod_{k=1}^{j+1} \lambda_{k, u_{k}}^{k}$ is an eigenvalue of $\tau$.

6.13. Lemma. Let $\left(f_{1}, \ldots, f_{j+1}\right)$ be of type $j+1$ w.r.t. $\bar{\triangle}_{j+1}\left(\mu_{Y}\right)$. Then there exists an integer $n$, and a factor $\tilde{Y}=Y_{j-1} \times \mathbb{T}^{n}$ of $Y$, such that if $p: Y \rightarrow \tilde{Y}$ is the factor map, then for $k=1, \ldots, j+1, f_{k}$ is cohomologous to a cocycle $\tilde{f}_{k} \circ p$. Furthermore, there exist functions $g_{1}, \ldots, g_{j+1}$, where $g_{k}: Y_{j-1}^{0} \rightarrow S^{1}$ is of type $j-1$, such that

$$
\left(\tilde{f}_{1} g_{1} \circ \pi_{j-1}, \ldots, \tilde{f}_{j+1} g_{j+1} \circ \pi_{j-1}\right)
$$

is of type $j+1$ w.r.t. $\bar{\triangle}_{j+1}\left(\mu_{\tilde{Y}}\right)$, and therefore Corollary 6.10 holds when replacing $f_{k}$ by $\tilde{f}_{k}$ and $Y$ by $\tilde{Y}\left(\pi_{j-1}\right.$ is the projection $\left.\tilde{Y} \rightarrow Y_{j-1}\right)$.

Proof. By Lemma 3.16 and Remark 3.18 we can find $J$ so that $H_{j} / J=\mathbb{T}^{n} \times \tilde{H}$, where $\tilde{H}$ is a compact totally disconnected abelian group (Lemma 3.16 can be carried out simultaneously for $\left.\left(f_{1}, \ldots, f_{j+1}\right)\right)$. Consider the system $W=Y_{j-1} \times_{\sigma_{j-1}}$ $\left(\mathbb{T}^{n} \times \tilde{H}\right)$ (abusing the notation). This system is a factor of $Y_{j}$. Therefore $Y_{j-1}(W)=$ $Y_{j-1}, W$ is a $(j-1)$-step pro-nilflow, and $Y_{j}(W)=W$ (this follows from the induction hypothesis in Theorem 6.1(5), and from Corollary 4.6). If $\tilde{H}$ is not trivial we get a contradiction to $Y_{j}(W)$ being an extension of $Y_{j-1}(W)$ by a connected abelian group for $j>1$ (this follows from the induction hypothesis in 6.1 (6) ). Now $Y$ can be presented as a skew product $Y=\tilde{Y} \times_{\tilde{\sigma}_{j-1}} J$ where

$$
\tilde{\sigma}_{j-1}(\tilde{y})=\bar{r}(\tilde{y}) \sigma_{j-1}\left(\pi_{j-1}(\tilde{y})\right) r(T \tilde{y}),
$$

where $r(\tilde{y})$ takes values in $H_{j}$. As in the proof of Lemma 6.8, there exists a character $\left(\chi_{1}, \ldots, \chi_{j+1}\right) \in \hat{J}^{j+1}$ such that

$$
\left(\tilde{f}_{1} \cdot \chi_{1}\left(\tilde{\sigma}_{j-1}\right), \ldots, \tilde{f}_{j+1} \cdot \chi_{j+1}\left(\tilde{\sigma}_{j-1}\right)\right)
$$

is of type $j+1$ w.r.t. $\bar{\triangle}_{j+1}\left(\mu_{\tilde{Y}}\right)$. Let $\tilde{\chi}_{k}$ be the lift of $\chi_{k}$ to a character of $H_{j}$. Then

Therefore

$$
\tilde{\chi}_{k}\left(\tilde{\sigma}_{j-1}(\tilde{y})\right)=\tilde{\chi}_{k}(\bar{r}(\tilde{y})) \tilde{\chi}_{k}\left(\sigma_{j-1}\left(\pi_{j-1}(\tilde{y})\right)\right) \tilde{\chi}_{k}(r(T \tilde{y}) .
$$

$$
\left(\tilde{f}_{1} \cdot \tilde{\chi}_{1}\left(\sigma_{j-1}\left(\pi_{j-1}\right)\right), \ldots, \tilde{f}_{j+1} \cdot \tilde{\chi}_{j+1}\left(\sigma_{j-1}\left(\pi_{j-1}\right)\right)\right)
$$

is of type $j+1$ w.r.t. $\bar{\triangle}_{j+1}\left(\mu_{\tilde{Y}}\right)$. 
6.14. Notation. If $U$ is an abelian group we denote rotation by an element of $U$ by $R_{u}$.

6.15. Lemma. Let $\left(f_{1}, \ldots, f_{j+1}\right)$ be of type $j+1$ w.r.t. $\bar{\triangle}_{j+1}\left(\mu_{Y}\right)$. Then there exists a factor of $Y$ which is a $(j-1)$-step nilflow $\tilde{Y}=(N / \Gamma, a)$, such that if $p: Y \rightarrow \tilde{Y}$ is the factor map, then

(1) there exists a function $\tilde{f}_{k}: N / \Gamma \rightarrow S^{1}$ such that $f_{k}$ is cohomologous to $\tilde{f}_{k} \circ p$;

(2) there exist functions $g_{1}, \ldots, g_{j+1}$, where $g_{k}: \pi_{j-1} N / \Gamma \rightarrow S^{1}$ is of type $j-1$, such that $\left(\tilde{f}_{1} g_{1} \circ \pi_{j-1}, \ldots, \tilde{f}_{j+1} g_{j+1} \circ \pi_{j-1}\right)$ is of type $j+1$ w.r.t. $\bar{\triangle}_{j+1}\left(\mu_{\tilde{Y}}\right)$ $\left(\pi_{j-1}\right.$ is the projection $\left.\tilde{Y} \rightarrow \pi_{j-1}(\tilde{Y})\right)$;

(3) Corollary 6.10 holds when replacing $f_{k}$ by $\tilde{f}_{k}$ and $Y$ by $\tilde{Y}$.

Proof. Recall the identification of $Y$ as a tower of abelian extensions $Y=H_{1} \times_{\sigma_{1}}$ $H_{2} \times \ldots \times_{\sigma_{j-1}} H_{j}$, where $Y_{i}(Y)=Y_{i-1}(Y) \times_{\sigma_{i-1}} H_{i}$, the group $H_{i}$ is an abelian group which is connected for $i>1$, and $\sigma_{i}$ is of type $i$. We would like to "replace" $H_{i}$ for $i \geq 2$ with a finite-dimensional torus $\mathbb{T}^{n_{i}}$ (and replace $H_{1}$ by a cyclic group) and get a system $C \times_{\sigma_{1}^{\prime}} \mathbb{T}^{n_{2}} \times_{\sigma_{2}^{\prime}} \ldots \times_{\sigma_{j-1}^{\prime}} \mathbb{T}^{n_{j}}$ that is a factor of $Y$ and therefore a nilflow (a pro-nilflow by the induction hypothesis in Theorem 6.1(5), and a nilflow by the induction hypothesis in 6.1(4) ), and if $p$ is the factor map, then $f_{k}$ is cohomologous to $\tilde{f}_{k} \circ p$.

We do this by decreasing induction on the index $i$. The case $i=j$ was proved in Lemma 6.13 Assume we have constructed a system $\tilde{Y}=H_{1} \times_{\sigma_{1}} \ldots \times H_{i} \times_{\sigma_{i}^{\prime}}$ $\mathbb{T}^{n_{i+1}} \times \ldots \times_{\sigma_{j-1}^{\prime}} \mathbb{T}^{n_{j}}$ that is a factor of $Y$ and satisfies (1)-(3). We may now forget the original system $Y$. By abuse of notation we replace $\tilde{Y}$ by $Y$, and $\tilde{f}_{k}$ by $f_{k}$. The cocycles $\sigma_{i}^{\prime}, \ldots, \sigma_{j-1}^{\prime}, g_{1}, \ldots, g_{j+1}$ are of type $<j$ and take values in finite-dimensional tori. Therefore there exists a finite-dimensional torus $\mathbb{T}^{n_{i}}$ with $\sigma_{l}^{\prime}$ for $l=i, \ldots, j-1$, cohomologous to functions $\tilde{\sigma}_{l}$, lifted from $Y_{l}^{\prime}:=H_{1} \times \ldots \times$ $H_{i-1} \times_{\sigma_{i-1}^{\prime}} \mathbb{T}^{n_{i}} \times \ldots \times_{\sigma_{l-1}^{\prime}} \mathbb{T}^{n_{l}}$, and $g_{k}$, for $k=1, \ldots, j+1$ cohomologous to $\tilde{g}_{k}$ lifted from $Y^{\prime}:=Y_{j+1}^{\prime}$. After reparametrization we may assume $\sigma_{l}^{\prime}$, for $l=i, \ldots, j-1$, is lifted from $Y_{l}^{\prime}$. Condition (2) remains valid if we can replace $g_{k}$ by $\tilde{g}_{k}$. Write $H_{i}=\mathbb{T}^{n_{i}} \times U$ (measurewise), where $U$ is a compact abelian group. The action of $T_{Y}$ commutes with rotation by an element in $U$, i.e., with

$$
R_{u^{\prime}}:\left(h_{1}, \ldots, h_{i-1}, t_{i}, u, t_{i+1}, \ldots, t_{j}\right) \rightarrow\left(h_{1}, \ldots, h_{i-1}, t_{i}, u+u^{\prime}, t_{i+1}, \ldots, t_{j}\right) .
$$

Indeed, $\sigma_{i}^{\prime}, \ldots, \sigma_{j-1}^{\prime}$ are not affected by translation in elements of $U$. Both $R_{u}, T_{Y}$ commute with rotation by an element $t \in \mathbb{T}^{n_{j}}$. Therefore

$$
\frac{f_{k}\left(R_{u}\left(\pi_{j-1} y, t_{j}+t\right)\right)}{f_{k}\left(R_{u}\left(\pi_{j-1} y, h\right)\right)} / \frac{f_{k}\left(\pi_{j-1} y, t_{j}+t\right)}{f_{k}\left(\pi_{j-1} y, t_{j}\right)}=\frac{f_{k, v}\left(R_{u} T y\right)}{f_{k, t}(T y)} / \frac{f_{k, t}\left(R_{u} y\right)}{f_{k, t}(y)} .
$$

By Theorem 3.6.

$$
f_{k}\left(R_{u} y\right) / f_{k}(y)
$$

is cohomologous to a cocycle lifted from $\pi_{j-1} Y$. By the same argument as in Lemma 6.10 it is cohomologous to a constant for $u$ in a neighborhood of zero in $U_{i}$. Now proceed as in Lemma 6.13 to obtain $\tilde{Y}$ (which will be a factor between $Y^{\prime}$ and $Y$ ). Applying the same procedure for $i=1$ gives the cyclic part. 
6.16. Remark. Let $f_{1}, \ldots, f_{j+1}$ be as in Lemma 6.15. By Remark 3.17 there are countably many possibilities for the groups $U_{i}$ and therefore countably many possibilities for the nilflow $N / \Gamma$ (up to isomorphism).

6.17. Let $(N / \Gamma, a)$ be the $(j-1)$-step nilflow from Lemma 6.15, and let $p: Y \rightarrow N / \Gamma$ be the projection. Let $N_{1}=N$, and $N_{l+1}=\left[N, N_{l}\right]\left(N_{j}=\{1\}\right)$. We will show that if the system $(N / \Gamma, a)$ has no finite nontrivial factors, then $N / \Gamma \times{ }_{\tilde{f}_{k}} S_{1}$ can be given the structure of a $j$-step nilflow. If the nilflow $(N / \Gamma, a)$ has a finite factor $C_{i}$, then $C_{i}$ is an abelian group of order $i$ for some integer $i$. The nilflow $\left(N / \Gamma, a^{i}\right)$ has finitely many ergodic components, and rotation by $a$ induces an isomorphism between them. Each ergodic component $X$ (with the action of $\left.a^{i}\right)$ is a $(j-1)$-step nilflow with no nontrivial finite factors. We will show that the system $X \times_{\tilde{f}_{k}} S^{1}$ is isomorphic to a $j$-step nilflow $(M / \Lambda, b)$. The system $N / \Gamma \times{\tilde{f}_{k}} S^{1}$ will then be isomorphic to a union of $i$ isomorphic $j$-step nilflows $C_{i} \times M / \Lambda$, with the action of $T$ given by: for $i-1>k \geq 0:(k, m \Lambda) \rightarrow(k+1, m \Lambda)$ and for $k=i-1:(i-1, m \lambda) \rightarrow(0, b m \Lambda)$. The group generated by $\{(0, m), T\}_{m \in M}$ is $j$-step nilpotent, and acts transitively on $C_{i} \times M / \Lambda$.

Assume $(N / \Gamma, a)$ has no finite nontrivial factors. The $r$-u.c.f. of $N / \Gamma$ is $Y_{r}(N / \Gamma)$ $=N / N_{r} \Gamma$ (this follows from Lemma 4.5). The system $(N / \Gamma, a)$ can be presented as an abelian extension of a $(j-2)$-step nilflow, i.e. $N / N_{j-1} \Gamma \times N_{j-1} /\left(N_{j-1} \cap \Gamma\right)$, and we may assume that $N$ is simply connected. The group $N_{j-1}$ is abelian, connected and simply connected ([L05]), therefore isomorphic to $\mathbb{R}^{m}$ for some $m$. Let the action of $T$ on $N / N_{2} \cong \mathbb{Z} \times \mathbb{R}^{n}$ (or $\mathbb{R}^{n}$ ) be given by translation by $\alpha$. Then equation (36) becomes: for any $c \in N_{j-1}, k=1, \ldots, j+1, y \in N / \Gamma$,

$$
\frac{\tilde{f}_{k}(c y)}{\tilde{f}_{k}(y)}=\lambda_{k, c} \frac{T f_{k, c}(y)}{f_{k, c}(y)} .
$$

6.18. Lemma. Let $(N / \Gamma, a)$ be the $(j-1)$-step nilflow from 6.17, Let $f_{1}, \ldots, f_{k}$ be functions in $B\left(N / \Gamma, S^{1}\right)$. Let $\left\{\lambda_{k, c}\right\}$ be a family of constants, $\left\{f_{k, c}\right\}_{c \in N_{j-1}}$ be a family of functions in $B\left(N / \Gamma, S^{1}\right)$ such that

$$
\frac{f_{k}(c y)}{f_{k}(y)}=\lambda_{k, c} \frac{f_{k, c}(a y)}{f_{k, c}(y)}
$$

for all $c \in N_{j-1}$. Then we can choose $f_{k, c}, \lambda_{k, c}$ in equation (38) such that

$$
f_{k, c_{1}}\left(k, c_{2} y\right) f_{k, c_{2}}(y) \sim f_{k, c_{1} c_{2}}(y) .
$$

Proof. It follows from Lemma 4.7

6.19. Lemma. Let $f_{k}, f_{k, c}, \lambda_{k, c}$ be from Lemma 6.18. Then for each $k=1, \ldots j+1$ there exists an integer matrix $L_{k}$, a neighborhood of zero $U \subset N_{j-1}$, and a family of functions $\left\{f_{k, c}\right\}_{c \in U}$, such that for all $c \in U$,

$$
\frac{f_{k}(c y)}{f_{k}(y)}=e^{2 \pi i\left\langle L_{k} \alpha, c\right\rangle} \frac{f_{k, c}(a y)}{f_{k, c}(y)} .
$$

Proof. It follows from Lemma 4.8

6.20. Corollary. Let $Y$ be a $(j-1)$-step pro-nilflow, $\left(f_{1}, \ldots, f_{j+1}\right)$ of type $j+1$ w.r.t. $\bar{\triangle}_{j+1}\left(\mu_{Y}\right)$. Then modulo quasi-coboundaries, $f_{k}$ belongs to a countable set. 
Proof. By Lemma 6.15, $f_{k}$ is cohomologous to a function $\tilde{f}_{k}$ lifted from a nilflow, and by Remark 6.16 there are countably many possibilities for this nilflow. Fix the nilflow. If $\left(\tilde{f}_{1}, \ldots, \tilde{f}_{j+1}\right),\left(\tilde{f}_{1}^{\prime}, \ldots, \tilde{f}_{j+1}^{\prime}\right)$ share the integer matrices $L_{1}, \ldots, L_{j+1}$ from Lemma 6.19, then by Corollary [3.9, $\tilde{f}_{k} / \tilde{f}_{k}^{\prime}$ is cohomologous to a function on $\pi_{j-1}(N / \Gamma)$. By Lemma 6.8 as a function on $\pi_{j-1} Y, \tilde{f}_{k} / \tilde{f}_{k}^{\prime}$ is of type $j-1$. By the induction hypothesis in Theorem 6.1(3b), $\tilde{f}_{k} / \tilde{f}_{k}^{\prime}$ belongs to a countable set modulo $\pi_{j-1} Y$ quasi-coboundaries. (If we use condition (2) in Lemma 6.15, then we can get that $\tilde{f}_{k} / \tilde{f}_{k}^{\prime}$ belongs to a countable set modulo $\pi_{j-1}(N / \Gamma)$ quasi-coboundaries.)

6.21. Lemma. Let $Y$ be a $(j-1)$-step pro-nilflow, and let $\left(f_{1}, \ldots, f_{l}\right)$ be of type $l$ w.r.t. $\bar{\triangle}_{l}\left(\mu_{Y}\right)$. Then for each $k=1, \ldots, l$ there exists a family of constants $\left\{\lambda_{k, u}\right\}_{u \in H_{j}}$, and a family of functions $\left\{f_{k, u}\right\}_{u \in H_{j}} \subset B\left(Y^{0}, S^{1}\right)$ such that

$$
\frac{f_{k}\left(\pi_{j-1} y, h+u\right)}{f_{k}\left(\pi_{j-1} y, h\right)}=\lambda_{k, u} \frac{T f_{k, u}(y)}{f_{k, u}(y)} \text {. }
$$

Proof. We use induction on $l$. The proof for $l \leq j+1$ is given in Corollary 6.10, Assume the statement holds for $l$; we show it for $l+1$. Let $\left(f_{1}, \ldots, f_{l+1}\right)$ be of type $l+1$ w.r.t. $\bar{\triangle}_{l+1}\left(\mu_{Y}\right)$, and let $M_{j-1, l+1}(0)=\left(M_{j-1, l+1}+\triangle_{l+1}\left(H_{j}\right)\right) \cap H_{j}^{l} \times\{0\}$. Let $\vec{u}=\left(u_{1}, \ldots, u_{l+1}\right) \in M_{j-1, l+1}(0)\left(u_{l+1}=0\right)$. Then

$$
\left(\frac{f_{1}\left(\pi_{j-1} y, h+u_{1}\right)}{f_{1}\left(\pi_{j-1} y, h\right)}, \ldots, \frac{f_{l}\left(\pi_{j-1} y, h+u_{l}\right)}{f_{l}\left(\pi_{j-1} y, h\right)}\right)
$$

is of type $l$ with respect to $\bar{\triangle}_{l}\left(\mu_{Y}\right)$, and by the induction hypothesis,

$$
\frac{f_{k}\left(\pi_{j-1} y, h+u_{k}+u\right) / f_{k}\left(\pi_{j-1} y, h+u\right)}{f_{k}\left(\pi_{j-1} y, h+u_{k}\right) / f_{k}\left(\pi_{j-1} y, h\right)}=\lambda_{k, u, u_{k}} \frac{T f_{k, u, u_{k}}(y)}{f_{k, u, u_{k}}(y)} .
$$

The projection of $M_{j-1, l+1}(0)$ on any coordinate $k \leq l$ is full. By Lemma 6.11 fixing $u, \lambda_{k, u, u_{k}}$ is multiplicative in $u_{k}$ in a neighborhood of zero in $H_{j}$. By Lemma 6.19, $\lambda_{k, u, u_{k}}$ is determined by an integer matrix. The same holds when interchanging the roles of $u$ and $u_{k}$. Therefore $\lambda_{k, u, u_{k}} \equiv 1$ in a neighborhood of zero in $H_{j}$. By Lemma 3.9

$$
\frac{f_{k}\left(\pi_{j-1} y, h+u\right)}{f_{k}\left(\pi_{j-1} y, h\right)}=g_{k, u}\left(\pi_{j-1} y\right) \frac{T f_{k, u}(y)}{f_{k, u}(y)} .
$$

As in Lemma 6.8 and Corollary 6.10, the functions $g_{k, u}\left(\pi_{j-1} y\right), f_{k, u}(y)$ can be chosen so that $g_{k, u}\left(\pi_{j-1} y\right)$ is a constant function on $Y_{j-1}$.

6.22. Corollary. We may replace the index $j+1$ in Lemmas/Corollaries 6.76 6.20 by the index $l$ for any $l \geq j+1$.

As a corollary we get

6.23. Proof of Theorem 6.1(3a): It follows from Lemma 6.15 and Corollary 6.22.

6.24. Proof of Theorem 6.1(3b): It follows from Corollaries 6.20 and 6.22.

6.25. We now fix $N / \Gamma$, a $(j-1)$-step nilflow as in 6.17. Let $\left(f_{1}, \ldots, f_{l}\right)$ be of type $l$ w.r.t. $\bar{\triangle}_{l}\left(\mu_{N / \Gamma}\right)$. We will show that the system $N / \Gamma \times_{f_{k}} S^{1}$ is isomorphic to a $j$-step nilflow. We have constructed families of functions $\left\{f_{k, c}\right\}_{c \in N_{j-1}}$ satisfying equation (38). 
6.26. Lemma. For any $c_{1}, c_{2} \in N_{j-1}, f_{k, c_{1}}$ and $f_{k, c_{2}}$ satisfying equation (38) we have

$$
\frac{f_{k, c_{1}}\left(c_{2} y\right)}{f_{k, c_{1}}(y)}=\frac{f_{k, c_{2}}\left(c_{1} y\right)}{f_{k, c_{2}}(y)} .
$$

Proof. It follows from Lemma 4.9

6.27. Consider the group

$$
\mathcal{G}=\left\{(n, f): n \in N, f \in B\left(N / \Gamma, S^{1}\right)\right\},
$$

with multiplication

$$
(n, f)(m, g)=\left(n m, f^{m} g\right), \quad\left(f^{m} g\right)(y)=f(m y) g(y) .
$$

The elements of the form $(1, C)$ where $C$ is a constant are in $Z(\mathcal{G})$, the center of $\mathcal{G}$. For $c \in N_{j-1}$, we can interpret equation (38) as

$$
\left[(a, f),\left(c, f_{c}\right)\right]=\left(1, \lambda_{c}\right) \in Z(\mathcal{G}) .
$$

We want to think of $f_{c}$ as elements of the $(j-1)$ th subgroup in the upper central series of $\mathcal{G}$, which will be a $j$-step nilpotent group. We now follow the derived series upward to construct for each element in $N$ a function $f_{k, n}$ that will satisfy good commuting relations with $\left(a, f_{k}\right)$.

6.28. Notation. For $n \in N_{i} \backslash N_{i+1},|n|=i$, and $f^{n}(y)=f(n y)$. We write $(n, f) \sim$ $(n, g)$ if $f \sim g$.

6.29. Proposition. Let $Y=(N / \Gamma, a)$ be a $(j-1)$-step nilflow. Let $\left(f_{1}, \ldots, f_{l}\right)$ be of type $j$ w.r.t. $\bar{\triangle}_{l}\left(\mu_{Y}\right)$. For $k=1, \ldots, l$ there exists a family of functions $\mathcal{F}_{k}=\left\{f_{k, n}\right\}_{n \in N}$, where $f_{k, n}: N / \Gamma \rightarrow S^{1}$ are measurable functions satisfying the following conditions:

(1) There exists a constant $\delta(k, a, n)$ such that

$$
\frac{f_{k}(n y)}{f_{k}(y)}=\delta(k, a, n) f_{k,[a, n]}(n a y) \frac{f_{k, n}(a y)}{f_{k, n}(y)} .
$$

(2) For any $c \in N_{j-1},|n|>1$,

$$
f_{k, c}^{n} f_{k, n}=f_{k, n}^{c} f_{k, c} .
$$

(3) There exists a constant $\delta\left(k, n_{1}, n_{2}\right)$ such that

$$
\frac{f_{k, n_{1}}\left(n_{2} y\right)}{f_{k, n_{1}}(y)}=\delta\left(k, n_{1}, n_{2}\right) f_{k,\left[n_{1}, n_{2}\right]}\left(n_{2} n_{1} y\right) \frac{f_{k, n_{2}}\left(n_{1} y\right)}{f_{k, n_{2}}(y)} .
$$

(4) $f_{k, n_{1} n_{2}}(y) \sim f_{k, n_{1}}\left(n_{2} y\right) f_{k, n_{2}}(y)$.

(5) If $\left(n_{1}, \ldots, n_{l}\right) \subset N^{l}$ preserves the ergodic components of $\tau_{l}(Y)$, then

$$
\frac{F\left(n_{1} y_{1}, \ldots, n_{l} y_{l}\right)}{F\left(y_{1}, \ldots, y_{l}\right)} \prod_{k=1}^{l} \bar{f}_{k, n_{k}}\left(y_{k}\right)
$$

is constant $\bar{\triangle}_{l}\left(\mu_{Y}\right)$ a.e.

Proof. The proof requires a series of Lemmas and their Corollaries and will be completed in 6.37 We prove this inductively, proceeding upward in the derived series of $N$. By Lemmas 6.19, 6.18, 6.26 and Corollary 6.12, conditions (11)-(4) hold for all $n \in N_{j-1}$. Condition (5) follows from the fact that $N_{j-1} \subset Z(N)$. Invariance 
under $\tau_{l}$ follows from equation (28) and Corollary 6.12. Invariance under $T_{l}$ follows from the fact that the function

$$
g\left(y_{1}, \ldots, y_{l}\right)=\frac{F\left(a y_{1}, \ldots, a y_{l}\right)}{F\left(y_{1}, \ldots, y_{l}\right)} \prod_{k=1}^{l} \bar{f}_{k}\left(y_{k}\right)
$$

is invariant under $\tau_{l}$ and therefore also under translation by $\left(n_{1}, \ldots, n_{l}\right)$. Thus

$$
\begin{aligned}
1=\frac{g\left(n_{1} y_{1}, \ldots, n_{l} y_{l}\right)}{g\left(y_{1}, \ldots, y_{l}\right)} & =\frac{F\left(a n_{1} y_{1}, \ldots, a n_{l} y_{l}\right)}{F\left(n_{1} y_{1}, \ldots, n_{l} y_{l}\right)} / \frac{F\left(a y_{1}, \ldots, a y_{l}\right)}{F\left(y_{1}, \ldots, y_{l}\right)} \prod_{k=1}^{l} \frac{\bar{f}_{k}\left(n_{k} y_{k}\right)}{\bar{f}_{k}\left(y_{k}\right)} \\
& =\frac{F\left(n_{1} a y_{1}, \ldots, n_{l} a y_{l}\right)}{F\left(a y_{1}, \ldots, a y_{l}\right)} / \frac{F\left(n_{1} y_{1}, \ldots, n_{l} y_{l}\right)}{F\left(y_{1}, \ldots, y_{l}\right)} \prod_{k=1}^{l} \frac{\bar{f}_{k, n_{k}}\left(a y_{k}\right)}{\bar{f}_{k, n_{k}}\left(y_{k}\right)} .
\end{aligned}
$$

Suppose we constructed $\left\{f_{n}\right\}$ for $n$ in $N_{i+1}(i+1>1)$ satisfying conditions (1) -(5). Let $n \in N_{i}$. Using conditions (1), (2), for $c \in N_{j-1}$ we have:

$$
\frac{f_{k}(n c y)}{f_{k}(c y)} \bar{f}_{k,[a, n]}(\text { nacy }) / \frac{f_{k}(n y)}{f_{k}(y)} \bar{f}_{k,[a, n]}(\text { nay })=\frac{f_{k, c}(n a y)}{f_{k, c}(a y)} / \frac{f_{k, c}(n y)}{f_{k, c}(y)} .
$$

By Corollary 3.9 there exist functions $f_{k, n}: N / \Gamma \rightarrow S^{1}$, and $g_{k, n}: N /\left(N_{j-1} \Gamma\right)$ $\rightarrow S^{1}$ such that

$$
\frac{f_{k}(n y)}{f_{k}(y)} \bar{f}_{k,[a, n]}(\text { nay })=g_{k, n}\left(\pi_{j-1} y\right) \frac{f_{k, n}(a y)}{f_{k, n}(y)} .
$$

We will show, in several steps, that one can choose $g_{k, n}, f_{k, n}$ so that $g_{k, n}\left(\pi_{j-1} y\right)$ is a constant function of $y$. Equation (40) would then mean that $f_{k}, f_{k, n}$ commute "nicely", i.e.,

$$
\left[\left(a, f_{k}\right),\left(n, f_{k, n}\right)\right] \sim\left([a, n], f_{k,[a, n]}\right) .
$$

6.30. Lemma. Let $c \in N_{j-1}$. There exists a constant $\delta(k, n, c) \in S^{1}$, which is multiplicative in $c$, so that

$$
f_{k, n}(c y) f_{k, c}(y)=\delta(k, n, c) f_{k, c}(n y) f_{k, n}(y) .
$$

Proof. By the induction hypothesis, $f_{k, c}^{[a, n]} f_{k,[a, n]}=f_{k,[a, n]}^{c} f_{k, c}$. Therefore

$$
\frac{f_{k, c}(n y) f_{k, n}(y)}{f_{k, c}(y) f_{k, n}(c y)}
$$

is a $T$-invariant function and therefore constant. Multiplicity in $c$ follows from Lemma 6.18.

6.31. Lemma. There exists a constant $C$ such that the $l$-tuple $\left(C g_{1, n}, \ldots, g_{l, n}\right)$ is of type l w.r.t. $\bar{\triangle}_{l}\left(\mu_{Y}\right)$.

Proof. Iterating equation (40) (using condition (4)) we get in a neighborhood of zero in $N_{i}$,

$$
\frac{f_{k}^{(k)}(n y)}{f_{k}^{(k)}(y)} \bar{f}_{k,\left[a^{k}, n\right]}\left(n a^{k} y\right)=g_{k, n}^{(k)}\left(\pi_{j-1} y\right) \frac{f_{k, n}\left(a^{k} y\right)}{f_{k, n}(y)} .
$$


Substituting in the functional equation (51) we get

$$
\begin{aligned}
& \prod_{k=1}^{l}\left(\left(g_{k, n}^{(k)}\left(\pi_{j-1} y_{k}\right) f_{k,\left[a^{k}, n\right]}\left(n a^{k} y_{k}\right) \frac{f_{k, n}\left(a^{k} y_{k}\right)}{f_{k, n}\left(y_{k}\right)}\right)\right. \\
& =\frac{F\left(a n y_{1}, \ldots, a^{l} n y_{l}\right) / F\left(n y_{1}, \ldots, n y_{l}\right)}{F\left(a y_{1}, \ldots, a^{l} y_{l}\right) / F\left(y_{1}, \ldots, y_{l}\right)} \\
& =\frac{F\left([a, n] n a y_{1}, \ldots,\left[a^{l}, n\right] n a^{l} y_{l}\right)}{F\left(n a y_{1}, \ldots, n a^{l} y_{l}\right)} \frac{G_{n}\left(a y_{1}, \ldots, a^{l} y_{l}\right)}{G_{n}\left(y_{1}, \ldots, y_{l}\right)},
\end{aligned}
$$

where

$$
G_{n}\left(y_{1}, \ldots, y_{l}\right)=F\left(n y_{1}, \ldots, n y_{l}\right) / F\left(y_{1}, \ldots, y_{l}\right) .
$$

By induction using condition (5),

$$
\frac{F\left([a, n] n a y_{1}, \ldots,\left[a^{l}, n\right] n a^{l} y_{l}\right)}{F\left(n a y_{1}, \ldots, n a^{l} y_{l}\right)} \prod_{k=1}^{l}\left(\bar{f}_{k,\left[a^{k}, n\right]}\left(n a^{k} y_{k}\right)\right)
$$

is constant $\bar{\triangle}_{l}\left(\mu_{Y}\right)$ a.e.

6.32. Corollary. For $k=1, \ldots, l, g_{k, n}$ is of type $j-1$. Therefore the set $\left\{g_{k, n}\right\}_{n \in N_{i}}$ modulo $\pi_{j-1} Y$-quasi-coboundaries is countable.

Proof. This follows from Lemma 6.8.

6.33. Corollary. Suppose for some $1 \leq r \leq j-1, g_{k, n}(y)=\tilde{g}_{k, n}\left(\pi_{r} y\right)$ for $k=$ $1, \ldots, l$. Then $\tilde{g}_{k, n}\left(\pi_{r} y\right)$ is of type $r$, and therefore the set $\left\{\tilde{g}_{k, n}\left(\pi_{r} y\right)\right\}_{n \in N_{i}}$ modulo $\pi_{r} Y$-quasi-coboundaries is countable.

6.34. Example. Before proceeding with the general proof we describe the proof for the case where $Y$ is a homogeneous space of a 3-step nilpotent group (i.e., $N_{4}=1$, $j-1=3), f_{k}: Y \rightarrow S^{1}$. Let $n, m \in N_{2}$. We use the facts that $[a, m] \in N_{3}$, therefore

$$
f_{k,[a, m]}^{n} f_{k, n} \sim f_{k, n}^{[a, m]} f_{k,[a, m]},
$$

and that for $m_{1}, m_{2} \in N_{3}$,

$$
f_{k, m_{1} m_{2}} \sim f_{k, m_{1}}^{m_{2}} f_{k, m_{2}} .
$$

Recall that by Corollary 6.32 there are countably many $g_{k, n}$ up to $\pi_{3} Y$-quasicoboundaries. Therefore there exists a neighborhood $U$ of zero in $N_{2}$ such that for any $n \in U$ there exists $m \in N_{2}$ so that $\left(g_{k, n m} / g_{k, m}\right)\left(\pi_{3} y\right)$ is a quasi-coboundary:

$$
\left(g_{k, n m} / g_{k, m}\right)\left(\pi_{3} y\right) \sim L_{k, n, m}\left(a \pi_{3} y\right) / L_{k, n, m}\left(\pi_{3} y\right) .
$$

Fix $n$ and replace $f_{k, m}(y)$ with $f_{k, m}(y) L_{k, n, m}\left(\pi_{3} y\right)$ (this does not affect the commutation relations with $\left(c, f_{k, c}\right)$ for $\left.c \in N_{3}\right)$. Then

$$
\begin{aligned}
\frac{f_{k}(n m y)}{f_{k}(m y)} & =\frac{f_{k}(n m y) / f_{k}(y)}{f_{k}(m y) / f_{k}(y)} \sim \frac{f_{k,[a, n m]}(n m a y)}{f_{k,[a, m]}(m a y)} \frac{f_{k, n m}\left(a m^{-1} m y\right) / f_{k, n m}\left(m^{-1} m y\right)}{f_{k, m}\left(a m^{-1} m y\right) / f_{k, m}\left(m^{-1} m y\right)} \\
& \sim f_{k,[n, a]}(\text { namy }) \frac{\tilde{f}_{k, n}(a m y)}{\tilde{f}_{k, n}(m y)}
\end{aligned}
$$


TAMAR ZIEGLER

where $\tilde{f}_{k, n}(y)=f_{k, n m}\left(m^{-1} y\right) / f_{k, m}\left(m^{-1} y\right)$. Therefore $g_{k, n}$ may be chosen to be constant (with a proper choice of $f_{k, n}$ ). Iterating, this holds for all $n \in N_{2}$. Observe that for $n, m \in N_{2}$,

$$
1=\frac{f_{k}(n m y)}{f_{k}(y)} / \frac{f_{k}(n m y)}{f_{k}(m y)} \frac{f_{k}(m y)}{f_{k}(y)} \sim \frac{f_{k, n m}(a y) / f_{k, n}(m a y) f_{k, m}(a y)}{f_{k, n m}(y) / f_{k, n}(m y) f_{k, m}(y)} .
$$

Now proceed as in Lemma 3.15 to modify $f_{k, n}$ so that $\delta(k, n, m)$ is multiplicative in $m, n \in N_{2}$, and $f_{k, n m} \sim f_{k, n}^{m} f_{k, n}$. As a corollary we get that for $c \in N_{3}, n \in N_{2}$ we have $f_{k, n}^{c} f_{k, c}=f_{k, c}^{n} f_{k, n}$ (same proof as Lemma 6.26).

Now let $n \in N_{1}=N$. We construct $f_{k, n}, g_{k, n}$ as in equation (40), and as in Corollary 6.32, $g_{k, n}$ is of type 3 . Therefore there exist constants $\{c(k, n, m)\}_{m \in N_{2}}$ and functions $h_{k, n, m}: \pi_{3} N / \Gamma \rightarrow S^{1}$ such that

$$
\frac{g_{k, n}\left(\pi_{3} m y\right)}{g_{k, n}\left(\pi_{3} y\right)}=c(k, n, m) \frac{h_{k, n, m}\left(\pi_{3} a y\right)}{h_{k, n, m}\left(\pi_{3} y\right)},
$$

where $c(k, n, m)$ is multiplicative in $m$, and there are countably many $g_{k, n}$ up to $\pi_{3} Y$-quasi-coboundaries. By Lemma 6.30 for $m \in N_{3}$ :

$$
f_{k, n}(m y) f_{k, m}(y)=\delta(k, n, m) f_{k, m}(n y) f_{k, n}(y),
$$

and $\delta(k, n, m)$ is multiplicative in $m$. We use this to establish commutation relations between $\left(n, f_{k, n}\right)$ and $\left(m, f_{k, m}\right)$ for $m \in N_{2}$ :

$$
\begin{aligned}
& \frac{T\left(\frac{f_{k, n}(m y)}{f_{k, n}(y)} / \frac{f_{k, m}(n y)}{f_{k, m}(y)} f_{k,[n, m]}(m n y)\right)}{\left(\frac{f_{k, n}(m y)}{f_{k, n}(y)} / \frac{f_{k, m}(n y)}{f_{k, m}(y)} f_{k,[n, m]}(m n y)\right)} \\
= & \delta(k, n,[a, m]) \delta(k, a,[m, n]) \delta(k, m,[n, a]) \frac{g_{k, n}\left(\pi_{3} m y\right)}{g_{k, n}\left(\pi_{3} y\right)} \\
= & \delta(k, n,[a, m]) \delta(k, a,[m, n]) \delta(k, m,[n, a]) c(k, n, m) \frac{h_{k, n, m}\left(\pi_{3} a y\right)}{h_{k, n, m}\left(\pi_{3} y\right)} .
\end{aligned}
$$

Thus for some eigenfunction $\psi_{k, n, m}\left(\pi_{2} y\right)$,

$$
\frac{f_{k, n}(m y)}{f_{k, n}(y)} \bar{f}_{k,[n, m]}(n m y)=\psi_{k, n, m}\left(\pi_{2} y\right) h_{k, n, m}\left(\pi_{3} y\right) \frac{f_{k, m}(n y)}{f_{k, m}(y)} .
$$

Let $m, n \in N$. Using the above equation we get

$$
\frac{g_{k, n m}\left(\pi_{3} y\right)}{g_{k, n}\left(\pi_{3} m y\right) g_{n}\left(\pi_{3} y\right)} h_{k, n,[m, a]}\left(\pi_{3} a m y\right) \sim \frac{T\left(f_{k, n m}(y) / f_{k, n}(m y) f_{k, m}(y)\right)}{f_{k, n m}(y) / f_{k, n}(m y) f_{k, m}(y)} .
$$

Similarly to Lemma 3.15 one shows, using Lemma 3.10, that $f_{k, n}, f_{k, m}$ can be modified so that for $m, n$ in a neighborhood of zero in $N$, there exists a function $K_{n, m}\left(\pi_{3} y\right)$ such that

$$
\frac{f_{k, n m}(y)}{f_{k, n}(m y) f_{k, m}(y)}=K_{n, m}\left(\pi_{3} y\right) .
$$

This implies that for $c \in N_{3}, \delta(k, n, c)$ is multiplicative for $n \in N$ : on the one hand,

$$
\frac{f_{k, n_{1} n_{2}}(c y)}{f_{k, n_{1} n_{2}}(y)}=\delta\left(k, n_{1} n_{2}, c\right) \frac{f_{k, c}\left(n_{1} n_{2} y\right)}{f_{k, c}(y)}
$$

while on the other hand,

$$
\frac{f_{k, n_{1} n_{2}}(c y)}{f_{k, n_{1} n_{2}}(y)}=\frac{f_{k, n_{1}}\left(n_{2} c y\right) f_{k, n_{2}}(c y)}{f_{k, n_{1}}\left(n_{2} y\right) f_{k, n_{2}}(y)}=\delta\left(k, n_{1}, c\right) \delta\left(k, n_{2}, c\right) \frac{f_{k, c}\left(n_{1} n_{2} y\right)}{f_{k, c}(y)} .
$$


Therefore for $m \in N_{2}, n \in N$ the constant $\delta(k, n,[a, m]) \delta(k, a,[m, n]) \delta(k, m,[n, a])$ is multiplicative in $m, n$. On the other hand, the constant

$$
\delta(k, n,[a, m]) \delta(k, a,[m, n]) \delta(k, m,[n, a]) c(k, n, m)
$$

is an eigenvalue of $T$, which is multiplicative in $m$ in a neighborhood of zero in $N_{2}$ and must therefore $\equiv 1$. This implies that $c(k, n, m)$ is multiplicative in $n$ and is therefore $\equiv 1$ (as a function of $n, c(k, n, m)$ is determined by an integer matrix). Thus $g_{k, n}$ is cohomologous to a function on $N / N_{2} \Gamma$, and we can choose $f_{k, n}$ so that $g_{k, n}$ is lifted from a function $\tilde{g}_{k, n}$ on $\pi_{2} Y$. Then $\left(n, f_{k, n}\right)\left(m, f_{k, m}\right)$ commute nicely for $m \in N_{2}$; namely $\left[\left(n, f_{k, n}\right),\left(m, f_{k, m}\right)\right] \sim\left([n, m], f_{[n, m]}\right)$. Let $n, m \in N$ be such that $\left(\tilde{g}_{k, n m} / \tilde{g}_{k, m}\right)\left(\pi_{2} y\right) \sim L_{k, n, m}\left(a \pi_{2} y\right) / L_{k, n, m}\left(\pi_{2} y\right)$ (by Corollary 6.33 there are countably many $\tilde{g}_{k, n}$ up to $\pi_{2} Y$-quasi-coboundaries). Replace $f_{k, m}(y)$ with $f_{k, m}(y) L_{k, n, m}\left(\pi_{2} y\right)$ (this does not affect the commutation relations with $\left(l, f_{k, l}\right)$ for $l \in N_{2}$ ). Now $g_{k, n m} / g_{k, m}$ is a constant. Computation (using the fact that $\left(n, f_{k, n}\right),\left(n m, f_{k, n m}\right)$ and $\left(\left[a, n^{-1}\right], f_{k,\left[a, n^{-1}\right]}\right)$ commute nicely) shows (as before):

$$
\frac{f_{k}(n m y)}{f_{k}(m y)}=\frac{f_{k}(n m y) / f_{k}(y)}{f_{k}(m y) f_{k}(y)} \sim f_{k,[n, a]}(n a m y) \frac{\tilde{f}_{k, n}(a m y)}{\tilde{f}_{k, n}(m y)} .
$$

Continue as in Lemma 3.15 to find that $f_{k, n m} \sim f_{k, n}^{m} f_{k, m}$.

We now proceed with the induction in Proposition 6.29. Basically we follow the same procedure.

6.35. Lemma. Let $n \in N_{i}$. The functions $g_{k, n}$ and $f_{k, n}$ from equation (40) can be chosen so that $g_{k, n}$ is a constant function on $Y$.

Proof. We already know that for $c \in N_{j-1}$ we have $f_{k, n}^{c} f_{k, c} / f_{k, c}^{n} f_{k, n}$ is a constant denoted $\delta(k, n, c)$. We use induction on $i+1 \leq r \leq j$ to acquire "good" commuting relations between $\left(n, f_{k, n}\right),\left(m, f_{k, m}\right)$ for $m \in N_{r}$, and to reduce the "level" of $g_{k, n}$. Assume that

(1) $g_{k, n}$ is lifted from $N / N_{r+1} \Gamma$ and is of type $r+1$.

(2) For $m \in N_{r+1}$, there exists a constant $\delta(k, n, m)$ such that

$$
f_{k, n}^{m} f_{k, m}=\delta(k, n, m) f_{k,[m, n]}^{m n} f_{k, m}^{n} f_{k, n} .
$$

(3) For $m \in N_{r+1}, n_{1}, n_{2} \in N_{i}$.

$$
\begin{aligned}
\delta\left(k, n_{1} n_{2}, m\right) f_{k,\left[m, n_{1} n_{2}\right]}= & \delta\left(k, n_{1},\left[n_{2}, m\right]\right) \delta\left(k, n_{1}, m\right) \delta\left(k, n_{2}, m\right) \\
& \left.\times f_{k,\left[\left[m, n_{1}\right], n_{2}\right]}^{\left[n_{1}, m\right]\left[n_{2}, m\right]} f_{k,\left[m, n_{2}\right]}^{\left[n_{1}, m\right]} f_{k,\left[m, n_{1}\right]}\right]
\end{aligned}
$$

and similarly for $\delta\left(k, n, m_{1} m_{2}\right)$ for $n \in N_{i}, m_{1}, m_{2} \in N_{r+1}$ (also for $\delta\left(k, a, m_{1} m_{2}\right)$, but this follows from the fact that $r>i$ and the induction hypothesis (10) in Proposition 6.29).

(4) $f_{k, n_{1} n_{2}}(y)=f_{k, n_{1}}^{n_{2}} f_{k, n_{2}}(y) K_{k, n_{1}, n_{2}}\left(\pi_{r+1}(y)\right)$.

Two equivalent forms of equation (40) are

$$
\begin{aligned}
& \left(a, f_{k}\right)\left(n, f_{k, n}\right)\left(1, \bar{g}_{k, n}\right)=\left([a, n], f_{k,[a, n]}\right)\left(n, f_{k, n}\right)\left(a, f_{k}\right), \\
& \left(n, f_{k, n}\right)^{-1}\left(a, f_{k}\right) \\
& =\left([a, n], f_{k,[a, n]}\right)\left(\left[[n, a], n^{-1}\right], f_{k,\left[[n, a], n^{-1}\right]}\right)\left(a, f_{k}\right)\left(1, g_{k, n}\right)\left(n, f_{k, n}\right)^{-1} .
\end{aligned}
$$


Using the above equations, and the fact that for any $m \in N_{r}$,

$$
\left[\left(a, f_{k}\right),\left(m, f_{k, m}\right)\right]=\left([a, m], \delta(k, a, m) f_{k,[a, m]}\right),
$$

for $m \in N_{r+1}$ (condition (2) ),

$$
\left[\left(n, f_{k, n}\right),\left(m, f_{k, m}\right)\right]=\left([n, m], \delta(k, n, m) f_{k,[n, m]}\right),
$$

and for any $m \in N$,

$$
\left(m, f_{k, m}\right)\left(1, g_{k, n}\right)=\left(1, g_{k, n}^{m^{-1}}\right)\left(m, f_{k, m}\right)
$$

we get for $m \in N_{r}$,

$$
\begin{aligned}
& \left(a, f_{k}\right)^{-1}\left(n, f_{k, n}\right)^{-1}\left(m, f_{k, m}\right)^{-1}\left([m, n], f_{k,[m, n]}\right)\left(n, f_{k, n}\right)\left(m, f_{k, m}\right)\left(a, f_{k}\right) \\
& =\left(n, f_{k, n}\right)^{-1}\left(m, f_{k, m}\right)^{-1}\left([m, n], f_{k,[m, n]}\right)\left(n, f_{k, n}\right)\left(m, f_{k, m}\right)\left(1, \Lambda(k, n, m) g_{k, n}^{m} \bar{g}_{k, n}\right)
\end{aligned}
$$

for some constant $\Lambda(k, n, m) \in S^{1}$. We explain this equality: we use the fact that we know how $\left(a, f_{k}\right)$ commutes with $\left(m, f_{k, m}\right),\left(n, f_{k, n}\right)$ for $m \in N_{r}, n \in N_{i}$, respectively. $\Lambda(k, n, m)$ is a product of functions of the type $\delta\left(k,\left[m^{-1},[n, a]\right]\right) f_{k,\left[m^{-1},[n, a]\right]}$, $\delta(k,[n,[m, a]]) f_{k,[n,[m, a]]}, \delta(k,[[m, n], a]) f_{k,[[m, n], a]}$ and more complicated commutators of involving $a, n, m$ and their inverses; $\Lambda(k, n, m)=\Pi \delta(k, \alpha, \beta) f_{k,[\alpha, \beta]}(y)$, where $\Pi[\alpha, \beta]=1$ since in $N$ we have $n^{-1} m^{-1}[m, n] n m=1$, therefore obviously $a^{-1} n^{-1} m^{-1}[m, n] n m a=n^{-1} m^{-1}[m, n] n m$. But as $[\alpha, \beta] \in N_{i+1}$ and for $v, w \in N_{i+1}$ we have $f_{v w} \sim f_{v}^{w} f_{w}, \Lambda(k, n, m)$ is a constant.

The induction hypothesis (3) applies for the commutators involved in the calculation of $\Lambda(k, n, m)$. Therefore for the purpose of calculating $\Lambda\left(k, n_{1} n_{2}, m\right)$ we can replace $\left(n_{1} n_{2}, f_{n_{1} n_{2}}\right)$ with $\left(n_{1} n_{2}, f_{n_{1}}^{n_{2}} f_{n_{2}}\right)$. Note that the function $g_{k, n}$ doesn't play a role in the calculation of $\Lambda(k, n, m)$, and therefore in calculating $\Lambda\left(k, n_{1} n_{2}, m\right)$, $\Lambda\left(k, n_{1}, m\right), \Lambda\left(k, n_{2}, m\right)$ we can replace the functions $g_{k, n_{1} n_{2}}, g_{k, n_{1}}, g_{k, n_{2}}$ with the constant function 1 in equation (46). Now using the induction hypothesis (3) we find that $\Lambda(k, n, m)$ is multiplicative in $n \in N_{i}$ and similarly in $m \in N_{r}$.

From equation (46) we get

$$
\frac{T\left(\frac{f_{k, n}(m y)}{f_{k, n}(y)} / \frac{f_{k, m}(n y)}{f_{k, m}(y)} f_{k,[n, m]}(m n y)\right)}{\left(\frac{f_{k, n}(m y)}{f_{k, n}(y)} / \frac{f_{k, m}(n y)}{f_{k, m}(y)} f_{k,[n, m]}(m n y)\right)}=\Lambda(k, n, m) \frac{g_{k, n}(m y)}{g_{k, n}(y)} .
$$

As $g_{k, n}=\tilde{g}_{k, n} \circ \pi_{r+1}$ for some function $\tilde{g}_{k, n}: \pi_{r+1} Y \rightarrow S^{1}$ of type $r+1$, for $m \in N_{r}$ there exist a constant $c(k, n, m)$ and a measurable function $h_{k, n, m}: \pi_{r+1} Y \rightarrow S^{1}$ such that

$$
\frac{\tilde{g}_{k, n}\left(\pi_{r+1} m y\right)}{\tilde{g}_{k, n}\left(\pi_{r+1} y\right)}=c(k, n, m) \frac{h_{k, n, m}\left(\pi_{r+1} a y\right)}{h_{k, n, m}\left(\pi_{r+1} y\right)}
$$

and $c(k, n, m)$ is multiplicative in $m$. Combining this with equation (47) we get that

$$
\frac{f_{k, n}(m y)}{f_{k, n}(y)} / \frac{f_{k, m}(n y)}{f_{k, m}(y)} f_{k,[n, m]}(m n y) \bar{h}_{k, n, m}\left(\pi_{r+1} y\right)
$$

is an eigenfunction of $T$. The corresponding eigenvalue $\Lambda(k, n, m) c(k, n, m)$ is a homomorphism from $N_{r}$ to a discrete set and is therefore constant and $\equiv 1$ (substitute $m=1)$. This implies that $c(k, n, m)$ is a multiplicative function of $n$. As $\tilde{g}_{k, n}$ is countably determined up to quasi-coboundaries $c(k, n, m) \equiv 1$ (recall that as a function of $m, c(k, n, m)$ is determined by an integer matrix). Therefore $\tilde{g}_{k, n}\left(m \pi_{r+1} y\right) / \tilde{g}_{k, n}\left(\pi_{r+1} y\right)$ is a $\pi_{r+1} Y$-coboundary for any $m \in N_{r}$; hence 
$\tilde{g}_{k, n}\left(\pi_{r+1} y\right)$ is $\pi_{r+1} Y$-cohomologous to a function on $\pi_{r} Y$. Therefore we can choose $g_{k, n}, f_{k, n}$ such that $g_{k, n}$ is lifted from a function on $\pi_{r} Y$, and

$$
f_{k, n}^{m} f_{k, m}=\delta(k, n, m) f_{k,[m, n]}^{m n} f_{k, m}^{n} f_{k, n} .
$$

We show by decreasing induction that for $m \in N_{s}$ for $s>i$,

$$
E_{k, n, m}=f_{k, m}^{n} f_{k, n} / f_{k,[m, n]}^{m n} f_{k, n}^{m} f_{k, m}
$$

is lifted from a function on $\pi_{r} Y$. For $m \in N_{r}$ this is clear by equation (48). Assuming it holds for $m \in N_{s+1}$, we show it holds for $m \in N_{s}$. By condition (44) it is clearly lifted from a function $\tilde{E}_{k, n, m}$ on $\pi_{r+1} Y$. A similar calculation to the one in equations (46), (47), using the induction hypothesis for $[a, m] \in N_{s+1}$, gives for $m \in N_{s}$ that

$$
T\left(f_{k, m}^{n} f_{k, n} / f_{k,[m, n]}^{m n} f_{k, n}^{m} f_{k, m}\right) /\left(f_{k, m}^{n} f_{k, n} / f_{k,[m, n]}^{m n} f_{k, n}^{m} f_{k, m}\right)
$$

is lifted from a function on $\pi_{r} Y$. As $\pi_{r+1} Y$ is an abelian extension of $\pi_{r} Y$ by some compact abelian group $Q$, by Lemma 3.10 there is a function $d_{k, n, m}: \pi_{r} Y \rightarrow S^{1}$ and a character $\chi_{k, n, m} \in \hat{Q}$ such that $\tilde{E}_{k, n, m}\left(\pi_{r+1} y\right)=d_{k, n, m}\left(\pi_{r} y\right) \chi_{k, n, m}\left(\pi_{r+1} y\right)$. As $s>i$, for $m_{1}, m_{2} \in N_{s}$, we have $f_{m_{1} m_{2}} \sim f_{m_{1}}^{m_{2}} f_{m_{2}}$. Therefore comparing $\tilde{E}_{k, n, m_{1} m_{2}}$ with $\tilde{E}_{k, n, m_{1}}^{m_{2}} \tilde{E}_{k, n, m_{2}}$ (the action of any element of $N$ on $\pi_{r+1} Y=\pi_{r} Y \times Q$ is a skew action and therefore $\left.\tilde{E}_{k, n, m}^{m_{2}}=\tilde{d}_{k, n, m} \chi_{k, n, m}\right)$ for some $\tilde{d}_{k, n, m}: \pi_{r} Y \rightarrow S^{1}$, we get that

$$
\chi_{k, n, m_{1} m_{2}}\left(\pi_{r+1} y\right) / \chi_{k, n, m_{1}}\left(\pi_{r+1} y\right) \chi_{k, n, m_{2}}\left(\pi_{r+1} y\right)
$$

is a function on $\pi_{r} Y$, and therefore $\equiv 1$. As $\hat{Q}$ is countable, $\chi_{k, n, m} \equiv 1$.

We now use this to show that for $n_{1}, n_{2} \in N_{i}$,

$$
T\left(f_{k, n_{1} n_{2}} / f_{k, n_{1}}^{n_{2}} f_{n_{2}}\right) /\left(f_{k, n_{1} n_{2}} / f_{k, n_{1}}^{n_{2}} f_{n_{2}}\right)
$$

is lifted from $\pi_{r} Y$. Using Lemma 3.10 and a similar argument to the one in Lemma 3.15 we find that the functions $f_{k, n}$ can be chosen so that for $n_{1}, n_{2} \in N_{i}$ there exists a function $K_{k, n_{1}, n_{2}}\left(\pi_{r} y\right)$ so that

$$
f_{k, n_{1} n_{2}}(y)=f_{k, n_{1}}^{n_{2}} f_{k, n_{2}}(y) K_{k, n_{1}, n_{2}}\left(\pi_{r} y\right) .
$$

To get the condition on $\delta(k, n, m)$ for $m \in N_{r}$ : on the one hand,

$$
\frac{f_{k, n_{1} n_{2}}(m y)}{f_{k, n_{1} n_{2}}(y)}=\delta\left(k, n_{1} n_{2}, m\right) f_{k,\left[n_{1} n_{2}, m\right]}\left(m n_{1} n_{2} y\right) \frac{f_{k, m}\left(n_{1} n_{2} y\right)}{f_{k, m}(y)},
$$

while on the other hand, as $K_{k, n_{1}, n_{2}}$ is invariant under translation by $m \in N_{r}$,

$$
\begin{aligned}
\frac{f_{k, n_{1} n_{2}}(m y)}{f_{k, n_{1} n_{2}}(y)}= & \frac{f_{k, n_{1}}\left(n_{2} m y\right) f_{k, n_{2}}(m y)}{f_{k, n_{1}}\left(n_{2} y\right) f_{k, n_{2}}(y)} \\
= & \delta\left(k, n_{1}, m\right) \delta\left(k, n_{2}, m\right) \delta\left(k, n_{1},\left[n_{2}, m\right]\right) f_{k,\left[n_{1}, m\right]}\left(m n_{1} n_{2} y\right) \\
& \times f_{k,\left[n_{2}, m\right]}\left(n_{1} m n_{2} y\right) f_{k,\left[n_{1},\left[n_{2}, m\right]\right]}\left(\left[n_{2}, m\right] n_{1} m n_{2} y\right) \frac{f_{k, m}\left(n_{1} n_{2} y\right)}{f_{k, m}(y)} .
\end{aligned}
$$

Finally, having good commutation relations between $\left(n, f_{k, n}\right),\left(m, f_{k, m}\right)$ for $n \in$ $N_{i}, m \in N_{i+1}$ (i.e., $\left.\left[\left(n, f_{k, n}\right),\left(m, f_{k, m}\right)\right]=\left([n, m], \delta(k, n, m) f_{k,[n, m]}\right)\right)$, we show that for $n \in N_{i}$, the functions $f_{k, n}, g_{k, n}$ can be chosen so that $g_{k, n}$ is constant. We already know it is lifted from a function $\tilde{g}_{k, n}$ on $\pi_{i+1} Y$ of type $i+1$, and there are only countably many of those up to $\pi_{i+1} Y$-quasi-coboundaries. For $n$ 
in a neighborhood of zero in $N_{i}$, let $m \in N_{i}$ be such that $\tilde{g}_{k, n m} / \tilde{g}_{k, m}$ is a quasicoboundary:

$$
\left(\tilde{g}_{k, n m} / \tilde{g}_{k, m}\right)\left(\pi_{i+1} y\right) \sim L_{k, n, m}\left(a \pi_{i+1} y\right) / L_{k, n, m}\left(\pi_{i+1} y\right) .
$$

Replace $f_{k, m}(y)$ with $f_{k, m}(y) L_{k, n, m}\left(\pi_{i+1} y\right)$ (this does not affect the commutation relations with $f_{k, p}$ for $\left.p \in N_{i+1}\right)$. Computation shows:

$$
\frac{f_{k}(n m y)}{f_{k}(m y)}=\frac{f_{k}(n m y) / f_{k}(y)}{f_{k}(m y) / f_{k}(y)} \sim f_{k,[n, a]}(n a m y) \frac{\tilde{f}_{k, n}(a m y)}{\tilde{f}_{k, n}(m y)}
$$

where

$$
\tilde{f}_{k, n}(y)=f_{k, n m}\left(m^{-1} y\right) / f_{k, m}\left(m^{-1} y\right) .
$$

We now use the fact that $f_{k,[n, a][m, a]} \sim f_{k,[n, a]}^{[m, a]} f_{k,[m, a]}$ to show that $f_{k, n m} / f_{k, n}^{m} f_{k, m}$ is an eigenfunction of $T$, and proceed as in Lemma 3.15 to find that $f_{k, n m} \sim$ $f_{k, n}^{m} f_{k, m}$.

6.36. Corollary. If $i>1$, then for any $c \in N_{j-1}, n \in N_{i}$,

$$
f_{k, n}(c y) f_{k, c}(y)=f_{k, c}(n y) f_{k, n}(y)
$$

(i.e., $\left.\left[\left(n, f_{k, n}\right),\left(c, f_{k, c}\right)\right]=(1,1)\right)$.

Proof. The quotient

$$
f_{k, n}(c y) f_{k, c}(y) / f_{k, c}(n y) f_{k, n}(y)
$$

is invariant under translation by $a$ and is therefore a constant $\delta(k, n, c)$ which is multiplicative in both coordinates. For $\gamma \in N_{j-1}, f_{\gamma}$ is an eigenfunction and therefore invariant under the action of $N_{i}$ for $i>1$. This implies that $\delta(k, n, c \gamma)=$ $\delta(k, n, c)$. Proceed as in Lemma 6.26.

6.37. Corollary. If $\left(n_{1}, \ldots, n_{l}\right) \subset N_{i}^{l}$ preserve the ergodic components of $\tau_{l}(Y)$, then

$$
\frac{F\left(n_{1} y_{1}, \ldots, n_{l} y_{l}\right)}{F\left(y_{1}, \ldots, y_{l}\right)} \prod_{k=1}^{l} \bar{f}_{k, n_{k}}\left(y_{k}\right)
$$

is constant $\bar{\triangle}_{l}\left(\mu_{Y}\right)$ a.e.

Proof. Both $\left(\left[a, n_{1}\right], \ldots,\left[a, n_{l}\right]\right)$, and $\left(\left[a, n_{1}\right], \ldots,\left[a^{l}, n_{l}\right]\right)$ preserve the ergodic components of $\tau_{l}$ (see 4.1), and as $g_{k, n}$ is constant for $n \in N_{i}$ the function in the left-hand side of equation (49) is invariant under $\tau_{l}, T_{l}$ (this is a calculation, using condition (10).

The proof of Proposition 6.29 is now complete.

6.38. Proposition. Let $Y=N / \Gamma$ be a $(j-1)$-step nilflow, and let $\left(f_{1}, \ldots, f_{l}\right)$ be of type $l$ w.r.t. $\bar{\triangle}_{l}\left(\mu_{Y}\right)$. Then for any $k=1, \ldots, l$ the system $Y \times_{f_{k}} S^{1}$ can be given the structure of a $j$-step nilflow.

Proof. Denote

$$
\mathcal{G}_{k}=\left\{\left(n, \psi f_{k, n}\right): n \in N, \psi \text { an eigenfunction, } f_{k, n} \in \mathcal{F}_{k}\right\},
$$

where $\mathcal{F}_{k}$ is defined in Proposition 6.29, $\mathcal{G}_{k}$ is a group under the multiplication

$$
(n, f)(m, g)=\left(n m, f^{m} g\right), \quad\left(f^{m} g\right)(y)=f(m y) g(y) .
$$


By Proposition 6.29(3), $\mathcal{G}_{k}$ is a $j$-step nilpotent group, and $\left(a, f_{k}\right) \in \mathcal{G}_{k}$. Endow $\mathcal{G}_{k}$ with the topology:

$$
\left(n_{i}, g_{i}\right) \rightarrow(n, g) \Longleftrightarrow n_{i} \rightarrow n, g_{i} \stackrel{L^{2}(N / \Gamma)}{\longrightarrow} g .
$$

$\mathcal{G}_{k}$ acts transitively and effectively on $X=N / \Gamma \times S^{1}$ by

$$
(n, f)(y, \zeta)=(n y, f(y) \zeta)
$$

By a theorem of Montgomery and Zippin (see GOV97, page 88, Theorem 4.3) it possesses a Lie group structure. (This type of construction is carried out in Me90 for the case $j=2$.)

6.39. Remark. It may also be possible to construct the nilflow by using the constants $\delta(k, n, m)$ as was done in R93, Le93] for 2-step nilpotent groups, and in [Z02 for 3 -step nilpotent groups.

6.40. Lemma. Let $Y=N / \Gamma$ be a $(j-1)$-step nilflow, and let $f: Y \rightarrow S^{1}$ be of type $j$. Then the system $Y \times{ }_{f} S^{1}$ can be given the structure of a $j$-step nilflow.

Proof. By definition, there exists an integer $l$ and integers $m_{1}, \ldots, m_{l}$, with $m_{k}=1$ for some $1 \leq k \leq l$, such that $\left(f^{m_{1}}, \ldots, f^{m_{l}}\right)$ is of type $l$ w.r.t. $\bar{\triangle}_{l}\left(\mu_{Y}\right)$. Now use Proposition 6.38

A similar proof gives:

6.41. Lemma. Let $Y=N / \Gamma$ be a $(j-1)$-step nilflow, and let $f, g: Y \rightarrow S^{1}$ be of type $j$. Then the system $Y \times_{f g} S^{1}$ can be given the structure of a $j$-step nilflow.

6.42. Corollary. Let $Y$ be a $(j-1)$-step pro-nilflow, and let $\left(f_{1}, \ldots, f_{l}\right)$ be of type $l$ w.r.t. $\bar{\triangle}_{l}\left(\mu_{Y}\right)$. Then for any $k=1, \ldots, l$ the system $Y \times_{f_{k}} S^{1}$ can be given the structure of a j-step pro-nilflow.

Proof. By Lemma 6.15 and Corollary 6.22, $f_{k}$ is cohomologous to a cocycle $\tilde{f}_{k}$ lifted from a $(j-1)$-step nilflow $(N / \Gamma, a)$. Furthermore, there exist $\left(g_{1}, \ldots, g_{l}\right)$ with $g_{k}$ of type $j-1$, such that $\left(\tilde{f}_{1} g_{1}, \ldots, \tilde{f}_{l} g_{l}\right)$ is of type $l$ w.r.t. $\bar{\triangle}_{l}\left(\mu_{N / \Gamma}\right)$. By Proposition 6.38, $N / \Gamma \times \times_{\tilde{f}_{k} g_{k}} S^{1}$ can be given the structure of a $j$-step nilflow. By Lemma 6.40, $N / \Gamma \times{ }_{g_{k}^{-1}} S^{1}$ can be given the structure of a $j$-step nilflow. By the construction in Proposition 6.38, $N / \Gamma \times \tilde{f}_{k} g_{k} g_{k}^{-1} S^{1}$ can be given the structure of a $j$-step nilflow.

6.43. Proof of 6.1 (4) Let $X=Y_{j}(X) \times_{\sigma} H$, where $H$ is a compact abelian group, and for any $\chi \in \hat{H}$, there exists $\left(\chi_{1}, \ldots, \chi_{l}\right) \in \hat{H}^{l}$, with $\chi=\chi_{k}$ for some $k$, and $\left(\chi_{1} \circ \sigma, \ldots, \chi_{l} \circ \sigma\right)$ of type $\vec{a}$ w.r.t. $\bar{\triangle}_{\vec{a}}\left(Y_{j}(X)\right)$. By Corollary 6.42 the system $X=Y_{j}(X) \times_{\chi \circ \sigma} S^{1}$ is isomorphic to a $j$-step pro-nilflow. By Pontryagin duality, $H \hookrightarrow\left(S^{1}\right)^{\hat{H}}$. The system $Y \times_{\sigma} H$ is therefore a "join" of factors of the form $Y \times_{\chi \circ \sigma} S^{1}$ where $\chi$ ranges over $\hat{H}$.

6.44. Lemma. Let $Y$ be a $(j-1)$-step pro-nilflow, and let $\left(f_{1}, \ldots, f_{l}\right)$ be of type $l$ w.r.t. $\bar{\triangle}_{l}\left(\mu_{Y}\right)$. If for some $k, \lambda_{k, u} \equiv 1$ (see Lemma 6.21) in a neighborhood of zero in $H_{j}$, then the system $Y \times_{f_{k}} S^{1}$ can be given the structure of a $(j-1)$-step pro-nilflow. 
Proof. By the induction hypothesis 6.1](6), $H_{j}$ is connected $(j \geq 1)$. Therefore we may choose $\lambda_{k, u} \equiv 1$ on $H_{j}$. By Corollary [3.9, $f_{k}$ is cohomologous to a cocycle $\tilde{f}_{k}$ lifted from $Y_{j-1}^{0}$. By Lemma 6.8, $\tilde{f}_{k}$ is of type $j-1$. By the induction hypothesis 6.1(4), $Y_{j-1} \times \tilde{f}_{k} S^{1}$ can be given the structure of a $(j-1)$-step pro-nilflow.

6.45. Lemma. Let $X=Y_{j}(X) \times_{\rho} H$, where $H$ is a compact abelian group, and $\rho$ is of type $j$. Then $H$ is connected.

Proof. We show that $\hat{H}$ has no elements of finite order. Suppose for some $\chi \in \hat{H}$, and some $l>0, \chi^{l}=1 . \chi \circ \rho$ satisfies equation (36). By Lemma 6.11 the function $\lambda_{k, u}$ is multiplicative in a neighborhood of zero in $H_{j} . \lambda_{k, u}^{l}$ is an eigenvalue, and as $H_{j}$ is connected, $\lambda_{k, u} \equiv 1$ in a neighborhood of zero. By Lemma 6.44 the system $Y_{j}^{\prime}=Y_{j}(X) \times_{\chi \circ \rho} S^{1}$, which is a factor of $X=Y_{j}(X) \times_{\rho} H$, can be given the structure of a $(j-1)$-step pro-nilflow. By Corollary 4.6. $Y_{j}\left(Y_{j}^{\prime}\right)=Y_{j}^{\prime}$, in contradiction to $Y_{j}(X)$ being the $j$-u.c.f. of $X$.

6.46. Lemma. Let $X$ be a group extension of $Y_{j}(X)$; i.e., $X=Y_{j}(X) \times_{\sigma} G$. Then $Y_{j+1}(X)$ is an abelian extension of $Y_{j}(X)$ by a cocycle of is type $j$ and therefore can be given the structure of a $j$-step pro-nilflow.

Proof. The proof is a straightforward generalization of Lemmas 9.1, 9.2 in [FuW96] (this is done for the case $j=3$ in [Z02]). We outline the steps. Any ergodic component of $\bar{\triangle}_{\vec{a}}\left(\mu_{X}\right)$ projects onto an ergodic component of $\bar{\triangle}_{\vec{a}}\left(\mu_{Y_{j}(X)}\right)$. The fact that $\tau_{\vec{a}}(T)$ and $T_{j+1}(T)$ commute implies that the Mackey groups associated with different ergodic components of $\bar{\triangle}_{\vec{a}}\left(\mu_{Y_{j}(X)}\right)$ are conjugate for a.e. ergodic components (Lemma 2.18). Denote by $\left[M_{\vec{a}}\right]$ the conjugacy class, where the group $M_{\vec{a}} \subset G^{j+1}$. One then uses the fact that the projection of $M_{\vec{a}}$ on any $j$ coordinates is full (i.e. $G^{j}$ ) to show that $[G, G]^{j+1} \subset M_{\vec{a}}$. More specifically one shows that there exists an abelian group $K_{\vec{a}}$ and homomorphisms $\psi_{\vec{a}, i}: G \rightarrow K_{\vec{a}}$ so that

$$
M_{\vec{a}}=\left\{\left(g_{1}, \ldots, g_{j+1} \mid \psi_{\vec{a}, i}\left(g_{1}\right) \ldots \psi_{\vec{a}, i}\left(g_{j+1}\right)=1\right\} .\right.
$$

We return to the average in (27). By Proposition 3.3 we can replace

$$
f_{1} \otimes \ldots \otimes f_{j+1}\left(y_{1}, g_{1}, \ldots, y_{j+1} g_{j+1}\right)
$$

by

$$
\int f_{1} \otimes \ldots \otimes f_{j+1}\left(y_{1}, g_{1} m_{1}, \ldots, y_{j+1}, g_{j+1} m_{j+1}\right) d m_{M_{\vec{a}}}\left(m_{1}, \ldots, m_{j+1}\right),
$$

where $d m_{M_{\vec{a}}}$ is the Haar measure on the Mackey group $M_{\vec{a}}$. As $[G, G]^{j+1} \subset M_{\vec{a}}$ we can replace $f_{k}$, for $k=1, \ldots, j+1$, by $\int f_{1}\left(y, g g^{\prime}\right) d m_{[G, G]}\left(g^{\prime}\right)$. Thus $Y_{j}(X) \times_{\rho}$ $G /[G, G]$ is characteristic for the scheme $\vec{a}$, for any $\vec{a}$.

Let $K_{0}=\bigcap_{k, \vec{a}} \operatorname{ker} \psi_{\vec{a}, k}$. Let $\tilde{G}=G / K_{0}$, and let $H=\tilde{G} /[\tilde{G}, \tilde{G}]$. Then similarly $Y_{j}(X) \times{ }_{\rho} H$ is characteristic for the scheme $\vec{a}$, for any $\vec{a}$. We will show that $\rho$ is of type $j$. Then by 6.43 it can be given the structure of a $j$-step pro-nilflow, and by Corollary 4.6 it is the $j+1$ universal characteristic factor.

Denote $Y=Y_{j}(X)$. Then by equation (26),

$$
\bar{\triangle}_{\vec{a}}\left(\mu_{X}\right)=\bar{\triangle}_{\vec{a}}\left(\mu_{Y}\right) \times m_{H}^{j+1}
$$

For each ergodic component of $\bar{\triangle}_{\vec{a}}\left(\mu_{Y}\right)$ the ergodic components of $\bar{\triangle}_{\vec{a}}\left(\mu_{X}\right)$ are given by the Mackey group $M_{\vec{a}} \subset H^{j+1}$. For a.e. ergodic component $W_{\vec{a}, y}$ (by the 
discussion in 4.1 the ergodic components of $\bar{\triangle}_{\vec{a}}\left(\mu_{Y}\right)$ are parametrized by $Y$ ) we have an $H^{j+1}$ - extension by the cocycle

$$
\tilde{\rho}_{\vec{a}}=\left(\rho^{\left(a_{1}\right)}\left(y_{1}\right), \rho^{\left(a_{2}\right)}\left(y_{2}\right), \ldots, \rho^{\left(a_{j+1}\right)}\left(y_{j+1}\right)\right): W_{\vec{a}, y} \rightarrow H^{j+1} .
$$

By Theorem 2.17 there exists a function $\varphi: W_{\vec{a}, y} \rightarrow H^{j+1}$ such that

$$
\varphi_{\vec{a}}\left(\tau_{\vec{a}}\left(y_{1}, \ldots, y_{j+1}\right)\right) \tilde{\rho}_{\vec{a}}\left(y_{1}, \ldots, y_{j+1}\right) \varphi_{\vec{a}}^{-1}\left(y_{1}, \ldots, y_{j+1}\right) \in M_{\vec{a}} .
$$

Applying the foregoing characterization of $M_{\vec{a}}$, there exists an abelian group $K_{\vec{a}}$ and homomorphisms $\psi_{\vec{a}, i}: H \rightarrow K_{\vec{a}}$ such that

$$
\prod_{k=1}^{j+1} \psi_{\vec{a}, k} \circ \rho^{\left(a_{k}\right)}\left(y_{k}\right)=F_{\vec{a}}\left(\tau_{\vec{a}}\left(y_{1}, \ldots, y_{j+1}\right)\right) F_{\vec{a}}^{-1}\left(y_{1}, \ldots, y_{j+1}\right),
$$

where

$$
F_{\vec{a}}\left(y_{1}, \ldots, y_{j+1}\right)=\prod_{k=1}^{j+1} \psi_{\vec{a}, i} \circ \varphi_{\vec{a}, i}\left(y_{1}, \ldots, y_{j+1}\right) \in K_{\vec{a}} .
$$

Let $\chi \in \hat{K}_{\vec{a}}$. Applying $\chi$ to equation (50) we get

$$
\prod_{k=1}^{j+1} \chi \circ \psi_{\vec{a}, k} \circ(\rho)^{\left(a_{k}\right)}\left(y_{k}\right)=\frac{\tau_{\vec{a}} F_{\vec{a}, y, \chi}\left(y_{1}, \ldots, y_{j+1}\right)}{F_{\vec{a}, y, \chi}\left(y_{1}, \ldots, y_{j+1}\right)},
$$

where $F_{\vec{a}, y, \chi}: W_{\vec{a}, y} \rightarrow S^{1}$. By ergodicity of $\tau_{\vec{a}}$ on $W_{\vec{a}, y}, F_{\vec{a}, y, \chi}$ is unique up to a constant multiple. By Proposition 3.13 there is a measurable choice of $F_{\vec{a}, y, \chi}$, so that equation (51) holds $\bar{\triangle}_{\vec{a}}\left(\mu_{Y}\right)$ a.e. Finally, as $\bigcap_{k=1}^{j+1}$ ker $\psi_{\vec{a}, k}=\{1\}$, the characters $\chi \circ \psi_{\vec{a}, k}$ where $k=1, \ldots, j+1, \vec{a} \in \mathbb{Z}^{j+1}$, and $\chi \in \hat{K}_{\vec{a}}$ span $\hat{H}$.

6.47. Proof of Theorem 6.1 (6). If $X$ is a $j$-step pro-nilflow, then $X$ is an abelian extension of $Y_{j}(X)$. By Corollary 4.6, $X=Y_{j+1}(X)$. By Lemma 6.46 it is an extension of $Y_{j}(X)$ by a cocycle of type $j$.

6.48. Corollary. Any j-step pro-nilflow $Y$ can be presented as a tower of abelian extensions $H_{1} \times_{\sigma_{1}} H_{2} \times \ldots \times_{\sigma_{j}} H_{j+1}$ where $\sigma_{k}$ for $k=1, \ldots, j$ is of type $k$. If in this presentation $H_{1}$ is a cyclic group, and for each $k>1, H_{k}$ is a finite-dimensional torus, then $Y$ is a nilflow.

Proof. The first part is clear. The second part follows from the construction in Proposition 6.38

6.49. Proof of 6.1(5). Let

$$
X=(N / \Gamma, T)=\left(\lim _{\leftarrow} N_{i} / \Gamma_{i}, a_{i}\right)
$$

be a $j$-step pro-nilflow, and let $W$ be a factor. Let $Y_{j}=\lim _{\leftarrow} M_{i} / \Lambda_{i}$ be the $j$-u.c.f. of $X$. Then $X=Y_{j} \times_{\sigma_{j}} H$ where $H$ is a compact abelian group, and $\sigma_{j}$ is of type $j$. Let $K$ be a compact abelian group of m.p.t.s acting on $X^{0}$ and commuting with the action of $T$. We show that $K$ commutes with the action of $N$. By Corollary 2.4, any $s \in K$ induces a map from $Y_{j}$ to itself, also denoted $s$ by abuse of notation. The action of $K$ is given by $s(y, h)=\left(s y, \rho_{s}(y, h)\right)$. We first show that $s$ preserves the skew product structure. As $s, T$ commute:

$$
\rho_{s}(T(y, h))=\rho_{s}(y, h)+\sigma_{j}(s y) .
$$


Denote

$$
F_{s}(y, h)=\rho_{s}(y, h)-h .
$$

Then

$$
T F_{s}(y, h)-F_{s}(y, h)=\sigma_{j}(s y)-\sigma_{j}(y) .
$$

Let $\chi$ be a character of $H$. Then

$$
\frac{\chi \circ \sigma_{j}(s y)}{\chi \circ \sigma_{j}(y)}=\frac{T \chi \circ F_{s}(y, h)}{\chi \circ F_{s}(y, h)} .
$$

As $\sigma_{j}$ is of type $j, \chi \circ \sigma_{j}$ is lifted from $M_{r} / \Lambda_{r}$ for some fixed $i$. Let $p: Y_{j} \rightarrow M_{r} / \Lambda_{r}$ be the projection. Then $\chi \circ \sigma_{j}=\tilde{\sigma}_{j} \circ p$. By the induction hypothesis $s$ acts as a translation by a group element on $M_{r} / \Lambda_{r}$ (we will denote this element $s$ by abuse of notation), commutes with the action of $M=\lim _{\leftarrow} M_{i}$ and therefore commutes with the action on $M_{r}$ on $M_{r} / \Lambda_{r}$. This implies that for any $m \in M_{r}$, $[s, m] \in Z\left(M_{r}\right) \cap \Lambda_{r}$. As $\tilde{\sigma}_{j}$ is of type $j$, by Proposition 6.29 there exist measurable functions $f_{\chi, s}: M_{r} / \Lambda_{r} \rightarrow S^{1}$, and such that

$$
\frac{\chi \circ \sigma_{j}(s y)}{\chi \circ \sigma_{j}(y)}=\frac{\tilde{\sigma}_{j}(s p y)}{\tilde{\sigma}_{j}(p y)}=f_{\chi,[s, a]}(p y) \frac{T f_{\chi, s}(p y)}{f_{\chi, s}(p y)} .
$$

But as $[s, a] \in Z\left(M_{r}\right) \cap \Lambda_{r}, f_{\chi,[s, a]}$ is an eigenfunction. Therefore

$$
\frac{\bar{f}_{\chi, s}(T p y) \chi \circ F_{s}(T(y, h))}{\bar{f}_{\chi, s}(p y) \chi \circ F_{s}(y, h)}=f_{\chi,[s, a]}(p y) .
$$

Therefore $f_{\chi, s}(p y) \chi \circ F_{s}(y, h)$ is a second-order eigenfunction $\psi\left(\pi_{3} y\right)$ (it is defined on $\pi_{3} Y$, a 2 -step nilflow). If $j \geq 3, F_{s}(y, h)$ depends only on $y$. Therefore

$$
\chi \circ \rho_{s}(y, h)=\chi(h) \psi\left(\pi_{3} y\right) f_{\chi, s}(p y) .
$$

This implies that the action of $k$ induces an action on $N_{i} / \Gamma_{i}$ for all $i$ that commutes with the action of $T$, and by [P73, Theorem 4.3] it commutes with the action of $N_{i}$ (the proof in $[\mathrm{P} 73$ is for $(N / \Gamma, a)$, where $N$ is connected, but the same proof holds in the case where $N$ is generated by $a$ and the connected component of the identity). We obtain the result inductively, using Proposition 4.12 and the fact that $X$ has generalized discrete spectrum $\bmod \mathcal{D}$ of finite type (see 4.10) and is therefore obtained from $W$ by a finite series of abelian extensions. If $j=2$, then $M_{r}$ is abelian, $f_{\chi,[s, a]}(p y)$ is a constant function of $y$,

$$
\frac{\bar{f}_{\chi, s}(T p y) \chi \circ F_{s}(T(y, h))}{\bar{f}_{\chi, s}(p y) \chi \circ F_{s}(y, h)}
$$

is an eigenfunction (defined on $\pi_{2} Y$, the Kronecker factor) and we proceed similarly.

6.50. Proof of Theorem 6.1(7). By 6.3, $Y_{j+1}(X)$ is an isometric extension of the factor $Y_{j}(X)$. By the discussion in 2.7. $Y_{j+1}(X)$ is of the form $Y_{j}(X) \times_{\sigma} G / L$, 
where $G / L$ is a homogeneous space of a compact metric group $G$. By Lemma 2.13 we may assume that the extension $X^{\prime}=Y_{j}(X) \times_{\sigma} G$ is an ergodic group extension. As $Y_{j}(X)$ is a factor of $Y_{j}\left(X^{\prime}\right)$, by Lemma 2.14, $X^{\prime}$ is group extension of $Y_{j}\left(X^{\prime}\right)$; i.e., $X^{\prime}=Y_{j}\left(X^{\prime}\right) \times_{\sigma^{\prime}} G^{\prime}$. By Corollary 2.4, the factor map $X^{\prime} \rightarrow Y_{j+1}(X)$ induces a map between their $(j+1)$-u.c.f.s. By 6.49, it is enough to show that $Y_{j+1}\left(X^{\prime}\right)$ has the structure of a $j$-step pro-nilflow. By Lemma 6.46 we are done.

6.51. Proof of Theorem 6.1(3c). By 6.42, $X=Y \times_{f_{k}} S^{1}$ can be given the structure of a $j$-step pro-nilflow; thus $Y_{j+1}(X)=X$. If $Y_{j}(X)=Y$, then by 6.47, $f_{k}$ is of type $j$. Otherwise, $Y$ is a proper factor of $Y_{j}(X)$ and therefore $Y_{j+1}(X)$ is an extension of $Y_{j}(X)$ by a proper closed subgroup $G$ of $S^{1}$. By 6.47 and 6.45, $G$ must be trivial; thus $Y_{j+1}(X)=Y_{j}(X)$, which is a $(j-1)$-step pro-nilflow. This implies that we can choose $f_{u, k}, \lambda_{u, k}$ in equation (36), with $\lambda_{u, k} \equiv 1$ (otherwise by Proposition 6.29 and the construction in Proposition 6.38, we increase the level of nilpotency). By Corollary [3.9, $f_{k}$ is cohomologous to a function $f_{k}^{\prime}$ on $Y_{j-1}(Y)$. The system $Y_{j-1} \times_{f_{k}^{\prime}} S^{1}$ is a factor of $X$ and therefore a $(j-1)$-step pro-nilflow. By the induction hypothesis 1.7(3c), $f_{k}^{\prime}$ is of type $j-1$; therefore $f_{k}$ is of type $j$.

6.52. Proof of Theorem 6.1(3d). If $f: Y \rightarrow H$ is of type $j$, then for any $\chi \in \hat{H}$ there exists $\left(\chi_{1}, \ldots, \chi_{l}\right) \in \hat{H}^{l}$ with $\chi=\chi_{k}$ for some $k$, and $\left(\chi_{1} \circ f, \ldots, \chi_{l} \circ f\right)$ is of type $\vec{a}$ w.r.t. $\bar{\triangle}_{\vec{a}}(Y)$. By 6.51, $\chi_{l} \circ f$ is of type $j$.

6.53. Proof of Theorem 6.1(3e). By Lemma 6.41, $Y \times_{f g} S^{1}$ can be given the structure of a pro-nilflow (it is clear from the proof of Lemma 6.15 that we can have the functions $f, g$ lifted from the same $(j-1)$-step nilflow). As in 6.51, $f g$ is of type $j$.

6.54. Proof of Theorem 6.1(8). This follows from 6.50 and 4.1.

\section{ACKNOWLEDGMENT}

Most of the ideas in this work appear in the author's Ph.D. thesis. I would like to thank my adviser Prof. Hillel Furstenberg for introducing me to ergodic theory, specifically to questions involving nonconventional ergodic averages, and for many fruitful discussions. I would also like to thank Benji Weiss, Shahar Mozes, Vitaly Bergelson and Emmanuel Lesigne for enlightening conversations and valuable remarks. I owe special thanks to Sasha Leibman for pointing out many inaccuracies in the early version of this paper.

\section{REFERENCES}

[Be87] Bergelson, V. Weakly mixing PET. Ergodic Theory Dynam. Systems 7 (1987), no. 3, 337-349. MR0912373 (89g:28022)

[BK96] Becker, H.; Kechris, S. The Descriptive Set Theory of Polish Group Actions. London Mathematical Society Lecture Note Series, 232. Cambridge University Press, Cambridge, 1996. MR 1425877 (98d:54068)

[Bo89] Bourgain, J. Pointwise ergodic theorems for arithmetic sets. Inst. Hautes Études Sci. Publ. Math. No. 69 (1989), 5-45. MR1019960 (90k:28030)

[CL84] Conze, J.P.; Lesigne, E. Théorèmes ergodiques pour des mesures diagonales. Bull. Soc. Math. France 112 (1984), no. 2, 143-175. MR0788966 (86i:28019)

[CL87] Conze, J.-P.; Lesigne, E. Sur un théorème ergodique pour des mesures diagonales. Probabilités, 1-31, Publ. Inst. Rech. Math. Rennes, 1987-1, Univ. Rennes I, Rennes, 1988. MR0989141(90a:28021) 
[CL88] Conze, J.P.; Lesigne, E. Sur un théorème ergodique pour des mesures diagonales. C. R. Acad. Sci. Paris Sér. I Math. 306 (1988), no. 12, 491-493. MR0939438 (89e:22012)

[Fu77] Furstenberg, H. Ergodic behavior of diagonal measures and a theorem of Szemerédi on arithmetic progressions. J. Analyse Math. 31 (1977), 204-256. MR0498471 (58:16583)

[FuW96] Furstenberg, H.; Weiss, B. A mean ergodic theorem for $1 / N \sum_{n=1}^{N} f\left(T^{n} x\right) g\left(T^{n^{2}} x\right)$. Convergence in ergodic theory and probability (Columbus, OH, 1993), 193-227, Ohio State Univ. Math. Res. Inst. Publ. 5, de Gruyter, Berlin, 1996. MR1412607 (98e:28019)

[GOV97] Gorbatsevich, V.V.; Onishchik, A.L.; Vinberg, E.B. Foundations of Lie Theory and Lie Transformation Groups. Springer-Verlag, Berlin, 1997. MR.1631937 (99c:22009)

[G01] Gowers, T. A new proof of Szemerédi's theorem. GAFA 11 (2001), 465-588. MR.1844079 (2002k:11014)

[GT04] Green, B.; Tao, T. The primes contain arbitrarily long arithmetic progressions. To appear Ann. of Math.

[HK01] Host, B.; Kra, B. Convergence of Conze-Lesigne averages. Ergodic Theory Dynam. Systems 21 (2001), no. 2, 493-509. MR 1827115 (2002d:28007)

[HK02] Host, B.; Kra, B. An odd Furstenberg-Szemerédi theorem and quasi-affine systems. J. Analyse Math. 86 (2002), 183-220. MR.1894481 (2003a:37003)

[HK02a] Host, B.; Kra, B. personal communication.

[HK05] Host, B.; Kra, B. Non-conventional ergodic averages and nilmanifolds. Ann. of Math. 161, 1 (2005) 397-488. MR2150389

[La54] Lazard, M. Sur les groupes nilpotents et les anneaux de Lie. Ann. Sci. Ecole Norm. Sup. (3) 71 (1954), 101-190. MR0088496 (19:529b)

[L98] Leibman, A. Polynomial sequences in groups. J. Algebra 201 (1998), no. 1, 189-206. MR.1608723 (99c:20044)

[L05] Leibman, A. Pointwise convergence of ergodic averages for polynomial sequences of translations on a nilmanifold. Ergodic Theory Dynam. Systems, (2005), no. 1, 201-213. MR2122919

[Le84] Lesigne, E. Résolution d'une équation fonctionnelle. Bull. Soc. Math. France 112 (1984), no. 2, 177-196. MR:0788967(86k:22018)

[Le87] Lesigne, E. Théorèmes ergodiques ponctuels pour des mesures diagonales. Cas des systèmes distaux. Ann. Inst. H. Poincaré Probab. Statist. 23 (1987), no. 4, 593-612. MR0928005 (89h:28032)

[Le89] Lesigne, E. Théorèmes ergodiques pour une translation sur une nilvariété. Ergodic Theory Dynam. Systems 9 (1989), no. 1, 115-126. MR0991492 (90e:58086)

[Le93] Lesigne, E. Équations fonctionelles, couplages de produits gauches et théorèmes ergodiques pour mesures diagonales. Bull. Soc. Math. France 121 (1993), no. 3, 315-351. MR:1242635 (94m:28029)

[Lu30] Lusin, N. Leçons sur les ensembles analytiques et leurs applications. Réimpression de l'edition de 1930. Chelsea Publishing Co., New York, 1972. MR0392465 (52:13282)

[Me90] Meiri, D. Generalized correlation series and nilpotent systems. M.Sc. thesis 1990.

[Pe83] Peterson, C. Ergodic Theory, Cambridge Studies in Advanced Mathematics, 2. Cambridge University Press, Cambridge, 1983. MR0833286 (87i:28002)

[P69] Parry, W. Ergodic properties of affine transformations and flows on nilmanifolds. Amer. J. Math. 91 (1969), 757-771. MR.0260975 (41:5595)

[P70] Parry, W. Dynamical systems on nilmanifolds. Bull. London Math. Soc. 2 (1970), 37-40. MR0267558 (42:2460)

[P73] Parry, W. Dynamical representations in nilmanifolds. Compositio Math. 26 (1973), 159174. MR 0320277 (47:8816)

[R93] Rudolph, D. Eigenfunctions of $T \times S$ and the Conze-Lesigne algebra. Ergodic theory and its connections with harmonic analysis (Alexandria, 1993), 369-432, London Math. Soc. Lecture Note Ser., 205, Cambridge Univ. Press, Cambridge, 1995 . MR1325712 (96k:28025)

[Sh96] Shah, N. Invariant measures and orbit closures on homogeneous spaces for actions of subgroups. Lie groups and ergodic theory (Mumbai, 1996), 229-271, Tata Inst. Fund. Res. Stud. Math., 14, Tata Inst. Fund. Res., Bombay, 1998. MR1699367 (2001a:22012)

[Z02] Ziegler, T. Non-conventional ergodic averages. Ph.D. thesis. Availiable on-line at http://www.math.ias.edu/ tamar/ 
[Z05] Ziegler, T. A non-conventional ergodic theorem for a nilsystem. Ergodic Theory Dynam. Systems, 25 (2005) no. 4, 1357-1370. MR2158410 (2006d:37009)

[Zi76] Zimmer, R. Extensions of ergodic group actions. Illinois J. Math. 20 (1976), no. 3, 373409. MR0409770(53:13522)

Department of Mathematics, The Ohio State University, 100 Math Tower, 231 West 18тh Avenue, Columbus, Ohio 43210

E-mail address: tamar@math.ohio-state.edu

Current address: School of Mathematics, The Institute of Advanced Study, Princeton, New Jersey 08540

E-mail address: tamar@math.ias.edu 